\title{
Filled elastomers: A theory of filler reinforcement based on hydrodynamic and interphasial effects
}

\author{
Taha Goudarzi, Daniel W. Spring, Glaucio H. Paulino, Oscar Lopez-Pamies \\ Department of Civil and Environmental Engineering, University of Illinois, Urbana-Champaign, IL 61801-2352, USA
}

\begin{abstract}
Experimental evidence has by now established that $i$ ) the hydrodynamic effect and $i i$ ) the presence of stiff interphases (commonly referred to as bound rubber) "bonding" the underlying elastomer to the fillers are the dominant microscopic mechanisms typically responsible for the enhanced macroscopic stiffness of filled elastomers. Yet, because of the technical difficulties of dealing with these fine-scale effects within the realm of finite deformations, the theoretical reproduction of the macroscopic mechanical response of filled elastomers has remained an open problem.

The object of this paper is to put forward a microscopic field theory with the capability to describe, explain, and predict the macroscopic response of filled elastomers under arbitrarily large nonlinear elastic deformations directly in terms of: $i$ ) the nonlinear elastic properties of the elastomeric matrix, $i i$ ) the concentration of filler particles, and $i i i$ ) the thickness and stiffness of the surrounding interphases. Attention is restricted to the prominent case of isotropic incompressible elastomers filled with a random and isotropic distribution of comparatively rigid fillers. The central idea of the theory rests on the construction of a homogenization solution for the fundamental problem of a Gaussian elastomer filled with a dilute concentration of rigid spherical particles bonded through Gaussian interphases of constant thickness, and on the extension of this solution to non-Gaussian elastomers filled with finite concentrations of particles and interphases by means of a combination of iterative and variational techniques.

For demonstration purposes, the theory is compared with full 3D finite-element simulations of the largedeformation response of Gaussian and non-Gaussian elastomers reinforced by isotropic distributions of rigid spherical particles bonded through interphases of various finite sizes and stiffnesses, as well as with experimental data available from the literature. Good agreement is found in all of these comparisons. The implications of this agreement are discussed.
\end{abstract}

Key words: finite strain; iterative homogenization methods; comparison medium methods; bound rubber; nanoparticulate composites

\section{Introduction and main results}

The addition of filler particles, even in small amounts, has long been known to significantly enhance the stiffness ${ }^{1}$ of elastomers. Over the past decades, a number of microscopic mechanisms have been conjectured to be responsible for such a profound resulting macroscopic reinforcement. Among these, arguably, there are two that experimental investigations have singled out to be dominant: $i$ ) the hydrodynamic and $i i$ ) the interphasial reinforcement effects (see, e.g., Heinrich et al., 2002; Berriot et al., 2002; Fukahori, 2007; Leblanc, 2010 and references therein). The so-called hydrodynamic effect refers to the fact that the addition of fillers

\footnotetext{
Email address: pamies@illinois.edu (Oscar Lopez-Pamies)

${ }^{1}$ The addition of filler particles is also known to significantly influence other mechanical properties of elastomers, including their fracture, abrasion, and rheological properties.
} 
to an elastomer results in a composite material whose macroscopic (overall or homogenized) mechanical properties are, by construction, some weighted average of the properties of the soft elastomer and the comparatively rigid fillers. The interphasial effect refers to the fact that the "anchoring" of the polymer chains of the elastomer onto the stiff filler particles forces the chains into conformations that are very different from those in the bulk, hence resulting in "interphases" (often referred to as bound rubber) of possibly several tens of nanometers in thickness that are substantially stiffer than the elastomer in the bulk.

Within the restricted context of linear elasticity, following the classical work of Einstein (1906) in the mathematically analogous setting of Stokes flow, Smallwood (1944) put forward a rigorous result that accounted for the hydrodynamic reinforcement generated by a dilute distribution of rigid spherical particles perfectly bonded (i.e., without interphases) to an isotropic incompressible elastomer. A number of exact and variational solutions were thereafter worked out that accounted for the hydrodynamic reinforcement generated by non-dilute distributions of spherical and non-spherical filler particles (see, e.g., Guth, 1945; Eshelby, 1957; Roscoe, 1973; Willis and Acton, 1976; Willis, 1977; Norris, 1985). Within the context of finite elasticity, because of the constitutive non-convexity of elastomers at finite deformations, the construction of corresponding results has proved more elusive. ${ }^{2}$ It is just recently that an exact result for the nonlinear elastic response of Gaussian (Neo-Hookean) elastomers reinforced by a dilute isotropic distribution of perfectly bonded rigid particles has been determined (Lopez-Pamies et al., 2013a). Building upon this fundamental dilute result, Lopez-Pamies et al. (2013b) have constructed a fairly general variational solution that allows to determine the hydrodynamic reinforcement generated by an isotropic distribution of rigid spherical particles perfectly bonded to non-Gaussian elastomers under arbitrarily large deformations.

The object of this paper is to build upon the work of Lopez-Pamies et al. (2013a; 2013b) to develop a microscopic field theory of filler reinforcement - accounting directly for both the hydrodynamic and the interphasial reinforcement effects - that can describe, explain, and predict the macroscopic response of filled elastomers undergoing arbitrarily large nonlinear elastic deformations ${ }^{3}$. Specifically, the focus is on the industrially prominent case of isotropic incompressible elastomers filled with a random and isotropic distribution of rigid particles of polydisperse sizes. This is accomplished by way of a twofold strategy. Roughly speaking, a solution is first constructed for the homogenized nonlinear elastic response of Gaussian elastomers filled with a dilute isotropic distribution of rigid particles and interphases. By means of a combination of iterative and variational techniques, this fundamental dilute result is then utilized to generate in turn a solution for the homogenized nonlinear elastic response of non-Gaussian elastomers filled with an isotropic distribution of rigid particles and interphases at finite concentrations. Here, it is relevant to remark that the reinforcement of materials (not just elastomers) via the addition of inclusions bonded through finite-size interphases is a subject that has received considerable attention over the last three decades, but almost exclusively within the restricted small-deformation contexts of linear elasticity (see, e.g., Walpole, 1978; Mikata and Taya, 1985; Qiu and Weng, 1991; Herve and Zaoui, 1993; Duan et al., 2006) and linear viscoelasticity (see, e.g., Hashin, 1991; Diani et al., 2013; Diani and Gilormini, 2014).

In this paper, for purposes of gaining further insight and of assessing the accuracy of the proposed theory, full 3D finite-element (FE) results are also generated for the large-deformation response of Gaussian and non-Gaussian elastomers reinforced by isotropic distributions of rigid spherical particles bonded through interphases of various finite sizes and stiffnesses. While this numerical approach for such a practically relevant problem seems simple enough, its presentation in the literature is not known to the authors.

The presentation of the work is organized as follows. Section 2 formulates the elastostatics problem that defines the overall nonlinear elastic response of filled elastomers, with a random distribution of rigid particles that are bonded to the elastomer through interphases, under finite deformations. Section 3 deals with the derivation of the first main result of this paper: the overall nonlinear elastic response of a Gaussian elastomer with stored-energy function $W_{m}=\mu_{m} / 2\left[I_{1}-3\right]$, filled with a dilute isotropic distribution of rigid particles that are bonded through interphases made up of a different Gaussian elastomer with stored-energy

\footnotetext{
${ }^{2}$ See, for example, the Introduction in Lopez-Pamies et al. (2013a) for a brief historical review on this problem.

${ }^{3}$ Dissipative processes such as viscous (see, e.g., Deepalekshmi and Thomas, 2014) and damage effects (see, e.g., Diani et al., 2009; Zhang et al., 2012; Gent and Park, 1984; Spring and Paulino, 2014) are not considered in this work.
} 
function $W_{i}=\mu_{i} / 2\left[I_{1}-3\right]$, is characterized by the effective stored-energy function

$$
\bar{W}=\frac{\mu_{m}}{2}\left[\bar{I}_{1}-3\right]+\frac{5 \mu_{m}}{4}\left[\bar{I}_{1}-3\right] c_{p}+\frac{5\left(\mu_{i}-\mu_{m}\right)\left(q_{1} \mu_{i}+q_{4} \mu_{m}\right) \mu_{m}}{4\left(q_{1} \mu_{i}^{2}+q_{2} \mu_{i} \mu_{m}+q_{3} \mu_{m}^{2}\right)}\left[\bar{I}_{1}-3\right] c_{i}
$$

to $O\left(c_{p}\right)$ and $O\left(c_{i}\right)$ in the concentration of particles $c_{p}$ and interphases $c_{i}$. Here, $\mu_{m}$ and $\mu_{i}$ stand for the initial shear moduli of the matrix and interphases, $\bar{I}_{1}=\overline{\mathbf{F}} \cdot \overline{\mathbf{F}}$ denotes the first principal invariant associated with the macroscopic deformation gradient $\overline{\mathbf{F}}$, and the coefficients $q_{1}, q_{2}, q_{3}, q_{4}$, which depend solely on the concentration ratio $c_{i} / c_{p}$, are given by

$$
\begin{aligned}
& q_{1}=38 k^{10}+225 k^{7}-336 k^{5}+200 k^{3}+48 \\
& q_{2}=89 k^{10}+75 k^{7}-168 k^{5}+100 k^{3}-96 \\
& q_{3}=48 k^{10}-300 k^{7}+504 k^{5}-300 k^{3}+48 \\
& q_{4}=\frac{4(k-1)^{3}\left(2 k^{3}+3\right)\left(4 k^{6}+16 k^{5}+40 k^{4}+55 k^{3}+40 k^{2}+16 k+4\right)}{k^{2}+k+1}
\end{aligned}
$$

with

$$
k=\left(1+\frac{c_{i}}{c_{p}}\right)^{1 / 3} .
$$

The fundamental dilute result (1) is utilized in Section 4 to generate in turn the following more general result: the overall nonlinear elastic response of a non-Gaussian elastomer with $I_{1}$-based stored-energy function $W_{m}=\Psi_{m}\left(I_{1}\right)$, filled with an isotropic distribution of rigid particles of polydisperse sizes and finite concentration $c_{p}$ that are bonded through interphases made up of a Gaussian elastomer with stored-energy function $W_{i}=\mu_{i} / 2\left[I_{1}-3\right]$ at finite concentration $c_{i}$, is characterized by the effective stored-energy function

$$
\bar{W}=\left(1-c_{p}-c_{i}\right) \Psi_{m}\left(\mathcal{I}_{1}\right)+\frac{\bar{\mu}_{0}}{2}\left[\bar{I}_{1}-3\right]-\left(1-c_{p}-c_{i}\right) \frac{\mu_{0}}{2}\left[\mathcal{I}_{1}-3\right],
$$

where

$$
\mathcal{I}_{1}=\left(\frac{c_{p} \bar{\mu}_{0}+\frac{\left(\mu_{i}-\bar{\mu}_{0}\right)\left(q_{1} \mu_{i}+q_{4} \bar{\mu}_{0}\right)}{q_{1} \mu_{i}^{2}+q_{2} \mu_{i} \bar{\mu}_{0}+q_{3} \bar{\mu}_{0}^{2}} c_{i} \bar{\mu}_{0}}{c_{p} \mu_{0}+\frac{\left(\mu_{i}-\mu_{0}\right)\left(q_{1} \mu_{i}+q_{4} \mu_{0}\right)}{q_{1} \mu_{i}^{2}+q_{2} \mu_{i} \mu_{0}+q_{3} \mu_{0}^{2}} c_{i} \mu_{0}}\right) \frac{\left[\bar{I}_{1}-3\right]}{1-c_{p}-c_{i}}+3
$$

and the variables $\bar{\mu}_{0}$ and $\mu_{0}$ are defined implicitly by the system of two coupled nonlinear algebraic equations

$$
\begin{aligned}
\mathcal{F}_{1}\left\{\bar{\mu}_{0}, \mu_{0}\right\}= & \frac{c_{i}\left[q_{4}^{2}-q_{4}\left(q_{1}+2 q_{2}+q_{3}\right)+q_{1} q_{3}\right]+c_{p}\left[q_{3}\left(2 q_{1}+q_{2}\right)-q_{4}\left(q_{2}+2 q_{3}\right)\right]}{\sqrt{c_{i}^{2}\left(q_{1}+q_{4}\right)^{2}+2 c_{i} c_{p}\left[q_{4}\left(2 q_{1}+q_{2}\right)-q_{1}\left(q_{2}+2 q_{3}\right)\right]+c_{p}^{2}\left(q_{2}^{2}-4 q_{1} q_{3}\right)}} \times \\
& \left\{\tanh ^{-1}\left[\frac{c_{i}\left(q_{4}-q_{1}\right)+c_{p} q_{2}+2\left(c_{p} q_{3}-c_{i} q_{4}\right) \frac{\bar{\mu}_{0}}{\mu_{i}}}{\sqrt{c_{i}^{2}\left(q_{1}+q_{4}\right)^{2}+2 c_{i} c_{p}\left[q_{4}\left(2 q_{1}+q_{2}\right)-q_{1}\left(q_{2}+2 q_{3}\right)\right]+c_{p}^{2}\left(q_{2}^{2}-4 q_{1} q_{3}\right)}}\right]-\right. \\
& \left.\tanh ^{-1}\left[\frac{c_{i}\left(q_{4}-q_{1}\right)+c_{p} q_{2}+2\left(c_{p} q_{3}-c_{i} q_{4}\right) \frac{\mu_{0}}{\mu_{i}}}{\sqrt{c_{i}^{2}\left(q_{1}+q_{4}\right)^{2}+2 c_{i} c_{p}\left[q_{4}\left(2 q_{1}+q_{2}\right)-q_{1}\left(q_{2}+2 q_{3}\right)\right]+c_{p}^{2}\left(q_{2}^{2}-4 q_{1} q_{3}\right)}}\right]\right\}- \\
& \frac{1}{2}\left(q_{3}+q_{4}\right) \ln \left[\frac{c_{i}\left(\bar{\mu}_{0}-\mu_{i}\right)\left(\mu_{i} q_{1}+q_{4} \bar{\mu}_{0}\right)-c_{p}\left(\mu_{i}^{2} q_{1}+\mu_{i} q_{2} \bar{\mu}_{0}+q_{3} \bar{\mu}_{0}^{2}\right)}{c_{i}\left(\mu_{0}-\mu_{i}\right)\left(\mu_{i} q_{1}+q_{4} \mu_{0}\right)-c_{p}\left(\mu_{i}^{2} q_{1}+\mu_{i} q_{2} \mu_{0}+q_{3} \mu_{0}^{2}\right)}\right]+ \\
& \left(q_{4}-\frac{c_{p}}{c_{i}} q_{3}\right) \ln \left[\left(1-c_{i}-c_{p}\right)^{5 / 2} \frac{\bar{\mu}_{0}}{\mu_{0}}\right]=0, \\
\mathcal{F}_{2}\left\{\bar{\mu}_{0}, \mu_{0}\right\}= & \Psi_{m}^{\prime}\left(\mathcal{I}_{1}\right)-\frac{\mu_{0}}{2}=0 .
\end{aligned}
$$


Section 4 includes a discussion of the key theoretical and practical features of this result. The basic details of the FE calculations are presented in Section 5. Section 6 provides sample predictions by the theoretical result (2) together with comparisons to corresponding FE solutions. Finally, Section 7 confronts the theoretical result (2) to experimental data and records some concluding remarks.

\section{The problem}

\subsection{Microscopic description of filled elastomers}

A filled elastomer is taken here to consist of a random distribution of rigid particles bonded to an elastomeric matrix through interphases of finite sizes; Fig. 1 shows a schematic illustration of this microscopic view. The domain occupied by the entire composite in its ground state is denoted by $\Omega$ and its boundary by $\partial \Omega$. Similarly, $\Omega_{m}, \Omega_{p}$, and $\Omega_{i}$ denote the domains occupied collectively by the matrix, the particles, and the interphases so that $\Omega=\Omega_{m} \cup \Omega_{p} \cup \Omega_{i}$ and their respective initial volume fractions (or concentrations) are given by $c_{m} \doteq\left|\Omega_{m}\right| /|\Omega|, c_{p} \doteq\left|\Omega_{p}\right| /|\Omega|$, and $c_{i} \doteq\left|\Omega_{i}\right| /|\Omega|$. We assume that the distribution of the particles is statistically uniform (i.e., translation invariant) and that their sizes and those of their surrounding interphases are much smaller that the size of $\Omega$.

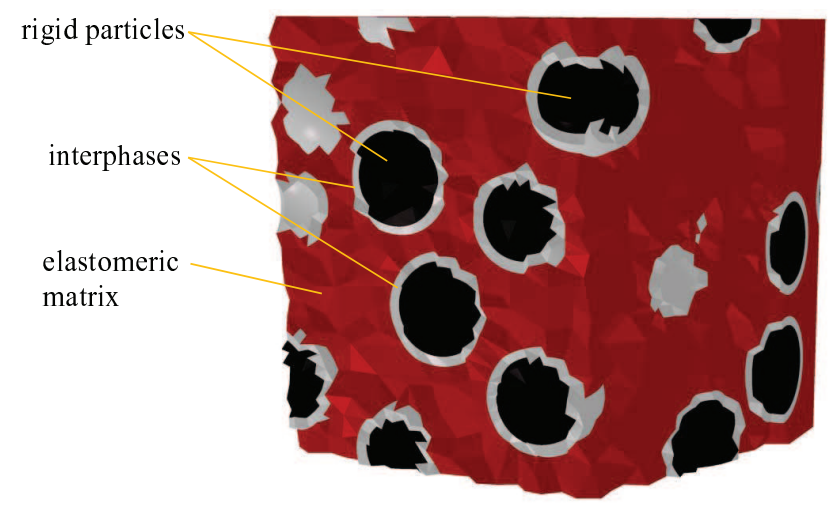

Figure 1: Schematic microscopic view of a filled elastomer.

Upon application of mechanical loads, the initial position vector $\mathbf{X}$ of a material point in $\Omega$ moves to a new position specified by $\mathbf{x}=\chi(\mathbf{X})$, where $\chi$ is a one-to-one mapping from $\Omega$ to the deformed configuration $\Omega^{\prime}$. We assume that $\chi$ is twice continuously differentiable, except possibly on the matrix/interphase and interphase/particles boundaries, where it is only required to be continuous. The associated deformation gradient is denoted by

$$
\mathbf{F}=\operatorname{Grad} \chi
$$

and its determinant by $J=\operatorname{det} \mathbf{F}$. The elastomeric matrix is considered to be a homogeneous nonlinear elastic solid with stored-energy function $W_{m}=W_{m}(\mathbf{F})$. Similarly, the rigid particles are homogeneous nonlinear elastic solids characterized by the stored-energy function

$$
W_{p}(\mathbf{F})=\left\{\begin{array}{ll}
0 & \text { if } \mathbf{F}=\mathbf{Q} \in \operatorname{Orth}^{+} \\
+\infty & \text { otherwise }
\end{array},\right.
$$

where $\mathrm{Orth}^{+}$stands for the set of all proper orthogonal second-order tensors. The interphases are taken to be nonlinear elastic solids as well, but need not be homogeneous. We write their stored-energy function as $W_{i}=W_{i}(\mathbf{X}, \mathbf{F})$. Given the above local constitutive descriptions, it follows that at each material point $\mathbf{X}$ in the undeformed configuration $\Omega$, the first Piola-Kirchhoff stress tensor $\mathbf{S}$ is given in terms of $\mathbf{F}$ simply by

$$
\mathbf{S}=\frac{\partial W}{\partial \mathbf{F}_{4}}(\mathbf{X}, \mathbf{F}),
$$


where

$$
W(\mathbf{X}, \mathbf{F})=\left[1-\theta_{p}(\mathbf{X})-\theta_{i}(\mathbf{X})\right] W_{m}(\mathbf{F})+\theta_{p}(\mathbf{X}) W_{p}(\mathbf{F})+\theta_{i}(\mathbf{X}) W_{i}(\mathbf{X}, \mathbf{F})
$$

with $\theta_{p}$ and $\theta_{i}$ denoting the characteristic functions of the spatial regions occupied by the particles and interphases: $\theta_{p}(\mathbf{X})=1$ if $\mathbf{X} \in \Omega_{p}$ and zero otherwise, and, likewise, $\theta_{i}(\mathbf{X})=1$ if $\mathbf{X} \in \Omega_{i}$ and zero otherwise.

\subsection{The macroscopic response}

In view of the assumed separation of length scales and statistical uniformity of the microstructure, the above-defined filled elastomer behaves macroscopically as a "homogenous" material. Its macroscopic or overall response is defined as the relation between the volume average of the first Piola-Kirchhoff stress $\mathbf{S}$ and the volume average of the deformation gradient $\mathbf{F}$ over the undeformed configuration $\Omega$ when the composite is subjected to affine boundary conditions (Hill, 1972). Consistent with our choice of $\mathbf{F}$ as the independent variable of the problem, we consider boundary conditions that are affine in the deformation, namely,

$$
\mathbf{x}=\overline{\mathbf{F}} \mathbf{X} \quad \text { on } \quad \partial \Omega,
$$

where the second-order tensor $\overline{\mathbf{F}}$ stands for a prescribed quantity. Granted the boundary data (7), the divergence theorem warrants that $|\Omega|^{-1} \int_{\Omega} \mathbf{F}(\mathbf{X}) \mathrm{d} \mathbf{X}=\overline{\mathbf{F}}$ and hence the derivation of the macroscopic response reduces to finding the average stress $\overline{\mathbf{S}} \doteq|\Omega|^{-1} \int_{\Omega} \mathbf{S}(\mathbf{X}) \mathrm{d} \mathbf{X}$ for a given $\overline{\mathbf{F}}$. The result can be conveniently written in a variational form as (Ogden, 1978)

$$
\overline{\mathbf{S}}=\frac{\partial \bar{W}}{\partial \overline{\mathbf{F}}}\left(\overline{\mathbf{F}}, c_{p}, c_{i}\right)
$$

where

$$
\bar{W}\left(\overline{\mathbf{F}}, c_{p}, c_{i}\right)=\min _{\mathbf{F} \in \mathcal{K}} \frac{1}{|\Omega|} \int_{\Omega} W(\mathbf{X}, \mathbf{F}) \mathrm{d} \mathbf{X},
$$

the effective stored-energy function, corresponds physically to the total elastic energy per unit undeformed volume stored in the filled elastomer. In these expressions, the concentrations $c_{p}$ and $c_{i}$ are utilized as explicit variables for later convenience and $\mathcal{K}$ denotes a sufficiently large set of kinematically admissible deformation gradient fields with prescribed volume average $\overline{\mathbf{F}}$.

\subsection{Filled elastomers with overall isotropic incompressible behavior: the basic case of spherical filler particles and constant-thickness Gaussian interphases}

The foregoing formulation is valid for arbitrary stored-energy functions for the elastomeric matrix, $W_{m}(\mathbf{F})$, and interphases, $W_{i}(\mathbf{X}, \mathbf{F})$, as well as for general classes of microstructures as characterized by $\theta_{p}(\mathbf{X})$ and $\theta_{i}(\mathbf{X})$. The focus of this work is on the prominent case of elastomeric matrices that are constitutively isotropic and incompressible and microstructures that are isotropic. Within this class of materials, we further restrict attention to those wherein the particles are spherical and the interphases are of constant thickness and made up of a Gaussian (Neo-Hookean) elastomer ${ }^{4}$. Specifically, we consider $I_{1}$-based stored-energy functions of the form

$$
W_{m}=\left\{\begin{array}{ll}
\Psi_{m}\left(I_{1}\right) & \text { if } J=\lambda_{1} \lambda_{2} \lambda_{3}=1 \\
+\infty & \text { otherwise }
\end{array} \quad \text { and } \quad W_{i}= \begin{cases}\frac{\mu_{i}}{2}\left[I_{1}-3\right] & \text { if } J=\lambda_{1} \lambda_{2} \lambda_{3}=1 \\
+\infty & \text { otherwise }\end{cases}\right.
$$

for the elastomeric matrix and interphases. In these expressions, $\lambda_{1}, \lambda_{2}, \lambda_{3}$ stand for the singular values of the deformation gradient tensor $\mathbf{F}, I_{1}=\mathbf{F} \cdot \mathbf{F}, \mu_{i}>0$ denotes the initial shear modulus of the interphases, and $\Psi_{m}$ is any non-negative function of choice satisfying the linearization conditions ${ }^{5}$

$$
\Psi_{m}(3)=0, \quad \Psi_{m}^{\prime}(3)=\frac{\mu_{m}}{2},
$$

\footnotetext{
${ }^{4}$ As elaborated further below, these geometric and constitutive idealizations prove sufficiently general for most filled elastomers, wherein the interphases are typically stiffer than the matrix.

${ }^{5}$ Throughout this paper, the notation $\Psi_{m}^{\prime}\left(I_{1}\right) \doteq \mathrm{d} \Psi_{m}\left(I_{1}\right) / \mathrm{d} I_{1}$ and $\Psi_{m}^{\prime \prime}\left(I_{1}\right) \doteq \mathrm{d}^{2} \Psi_{m}\left(I_{1}\right) / \mathrm{d} I_{1}^{2}$ is used for convenience.
} 
where $\mu_{m}$ denotes the initial shear modulus of the elastomeric matrix, and the strong ellipticity conditions (Zee and Sternberg, 1983)

$$
\Psi_{m}^{\prime}\left(I_{1}\right)>0, \quad \Psi_{m}^{\prime}\left(I_{1}\right)+2\left[I_{1}-\lambda_{\alpha}^{2}-2 \lambda_{\alpha}^{-1}\right] \Psi_{m}^{\prime \prime}\left(I_{1}\right)>0 \quad(\alpha=1,2,3) \quad \forall I_{1} \geq 3 .
$$

Stored-energy functions of the form (10) 1 with (11)-(12) are generalization of the classical Gaussian energy $\Psi_{m}\left(I_{1}\right)=\mu_{m} / 2\left[I_{1}-3\right]$ that have been shown to describe reasonably well the response of a wide variety of elastomers over large ranges of deformations (see, e.g., Arruda and Boyce, 1993; Gent, 1996; Lopez-Pamies, 2010a). A further merit of these types of constitutive models is that they are derivable from microscopic considerations (see, e.g., Beatty, 2003).

Owing to the assumed constitutive isotropy and incompressibility of the matrix material $(10)_{1}$, interphases $(10)_{2}$, and rigid particles (4), and the assumed isotropy of the microstructure, the resulting overall elastic response is isotropic and incompressible. This implies that the effective stored-energy function $\bar{W}$ in this case depends on the macroscopic deformation gradient $\overline{\mathbf{F}}$ only through its singular values $\bar{\lambda}_{1}, \bar{\lambda}_{2}, \bar{\lambda}_{3}$ and becomes unbounded for non-isochoric deformations when $\bar{J} \doteq \operatorname{det} \overline{\mathbf{F}}=\bar{\lambda}_{1} \bar{\lambda}_{2} \bar{\lambda}_{3} \neq 1$. Accordingly, the result (9) can be simply written as a symmetric function of $\bar{\lambda}_{1}, \bar{\lambda}_{2}, \bar{\lambda}_{3}$ subject to the constraint $\bar{\lambda}_{1} \bar{\lambda}_{2} \bar{\lambda}_{3}=1$. Alternatively, in this work, we shall find it more convenient to write the effective stored-energy function (9) as a

function of the two principal invariants $\bar{I}_{1}=\overline{\mathbf{F}} \cdot \overline{\mathbf{F}}=\bar{\lambda}_{1}^{2}+\bar{\lambda}_{2}^{2}+\bar{\lambda}_{3}^{2}$ and $\bar{I}_{2}=\overline{\mathbf{F}}^{-T} \cdot \overline{\mathbf{F}}^{-T}=\bar{\lambda}_{1}^{2} \bar{\lambda}_{2}^{2}+\bar{\lambda}_{1}^{2} \bar{\lambda}_{3}^{2}+\bar{\lambda}_{2}^{2} \bar{\lambda}_{3}^{2}$ in the form

$$
\bar{W}\left(\overline{\mathbf{F}}, c_{p}, c_{i}\right)=\left\{\begin{array}{ll}
\bar{\Psi}\left(\bar{I}_{1}, \bar{I}_{2}, c_{p}, c_{i}\right) & \text { if } \bar{J}=1 \\
+\infty & \text { otherwise }
\end{array} .\right.
$$

The object of this paper reduces hence to generating a solution for the finite branch $\bar{\Psi}$ of the effective stored-energy function (13). Paralleling previous work on filled elastomers without interphases (LopezPamies et al., 2013a; 2013b), as already alluded to in Section 1, our strategy to generate such a solution involves two main steps. In the first step, presented in Section 3, we work out a solution for the fundamental limiting case of Gaussian elastomers filled with a dilute concentration of particles and interphases. This dilute solution for Gaussian elastomers is then utilized in a second step, presented in Section 4, to work out in turn a solution for non-Gaussian elastomers filled with a finite concentration of particles and interphases. In order to assist the presentation of the results, the unbounded branch of the energies (10) and (13) is omitted in most of the remainder of the analysis.

\section{Dilute concentration of particles and interphases in Gaussian elastomers}

In this section, we derive an asymptotic solution for the effective stored-energy function $\bar{\Psi}$, as defined by (13) with (9), of filled elastomers in the limit when the filler particles and surrounding interphases are present in dilute concentrations, as $c_{p} \rightarrow 0+$ and $c_{i} \rightarrow 0+$, and the behavior of the matrix is characterized by the Gaussian stored-energy function

$$
\Psi_{m}\left(I_{1}\right)=\frac{\mu_{m}}{2}\left[I_{1}-3\right]
$$

where, again, $\mu_{m}$ stands for the shear modulus of the elastomeric matrix; recall that the interphase is also comprised of a Gaussian elastomer but with different shear modulus $\mu_{i}$.

Assuming that the particles are "well separated" and thus do not interact with one another in the limit as $c_{p} \rightarrow 0+$ and $c_{i} \rightarrow 0+$, the effective stored-energy function $\bar{\Psi}$ for a dilute suspension of particles is expected to agree identically with the total elastic energy per unit undeformed volume of an infinitely large matrix containing just a single spherical particle. This single-particle problem, schematically depicted in Fig. 2, is now taken up. In the calculations that follow, we shall denote the radius of the particle by $r$ and the initial thickness of the interphase by $t$. 


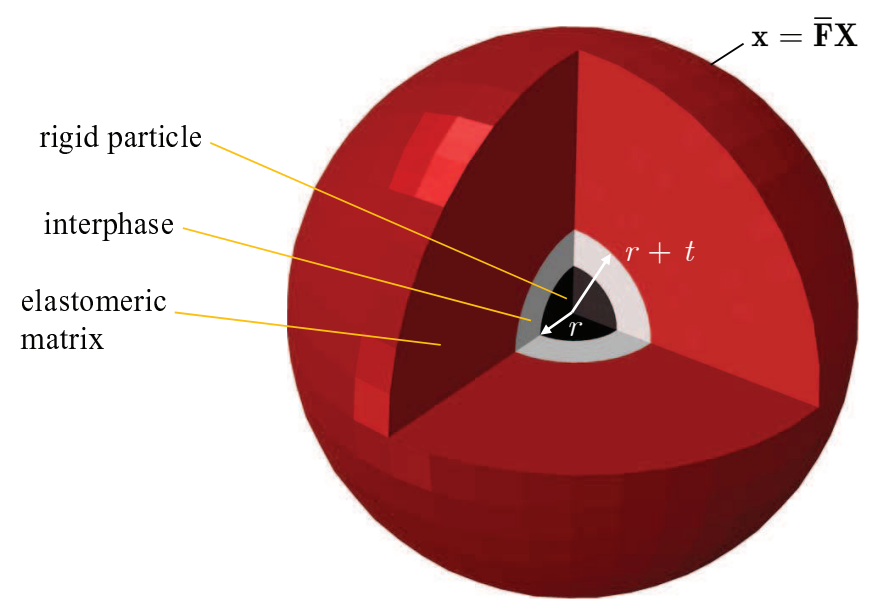

Figure 2: Schematic of the single-particle problem: a rigid spherical particle of radius $r$ is bonded through an interphase of thickness $t$ to a matrix of infinite extent that is subjected to the affine boundary condition $\mathbf{x}=\overline{\mathbf{F}} \mathbf{X}$. The matrix and interphase are both Gaussian elastomers with shear moduli $\mu_{m}$ and $\mu_{i}$, respectively.

\subsection{The exact solution in the small-deformation limit}

It is instructive to begin by examining the small deformation limit as the applied macroscopic deformation $\overline{\mathbf{F}} \rightarrow \mathbf{I}$ with $\operatorname{det} \overline{\mathbf{F}}=1$. In this limit, the elasticity problem (9) for the case of the single spherical particle admits an exact closed-form solution; for clarity of presentation, the relevant calculations are provided in Appendix A. The result for the finite branch $\bar{\Psi}$ of the effective stored-energy function reads as

$$
\begin{aligned}
\bar{\Psi}\left(\bar{I}_{1}, \bar{I}_{2}, c_{p}, c_{i}\right) & =\mu_{m} \operatorname{tr} \bar{\varepsilon}^{2}+\frac{5 \mu_{m}}{2} c_{p} \operatorname{tr} \bar{\varepsilon}^{2}+\frac{5\left(\mu_{i}-\mu_{m}\right)\left(q_{1} \mu_{i}+q_{4} \mu_{m}\right) \mu_{m}}{2\left(q_{1} \mu_{i}^{2}+q_{2} \mu_{i} \mu_{m}+q_{3} \mu_{m}^{2}\right)} c_{i} \operatorname{tr} \bar{\varepsilon}^{2} \\
& =\bar{\mu}^{\mathrm{dil}} \operatorname{tr} \bar{\varepsilon}^{2}
\end{aligned}
$$

to leading order in the deformation measure $\bar{\varepsilon} \doteq\left(\overline{\mathbf{F}}+\overline{\mathbf{F}}^{T}-2 \mathbf{I}\right) / 2$ and to $O\left(c_{p}\right)$ and $O\left(c_{i}\right)$, where the coefficient

$$
\bar{\mu}^{\text {dil }} \doteq \mu_{m}+\frac{5 \mu_{m}}{2} c_{p}+\frac{5\left(\mu_{i}-\mu_{m}\right)\left(q_{1} \mu_{i}+q_{4} \mu_{m}\right) \mu_{m}}{2\left(q_{1} \mu_{i}^{2}+q_{2} \mu_{i} \mu_{m}+q_{3} \mu_{m}^{2}\right)} c_{i}
$$

has been introduced for later reference and

$$
\begin{aligned}
& q_{1}=38 k^{10}+225 k^{7}-336 k^{5}+200 k^{3}+48 \\
& q_{2}=89 k^{10}+75 k^{7}-168 k^{5}+100 k^{3}-96 \\
& q_{3}=48 k^{10}-300 k^{7}+504 k^{5}-300 k^{3}+48 \\
& q_{4}=\frac{4(k-1)^{3}\left(2 k^{3}+3\right)\left(4 k^{6}+16 k^{5}+40 k^{4}+55 k^{3}+40 k^{2}+16 k+4\right)}{k^{2}+k+1}
\end{aligned}
$$

with

$$
k=1+\frac{t}{r}=\left(1+\frac{c_{i}}{c_{p}}\right)^{1 / 3}
$$

Expression (16) corresponds to the effective shear modulus of an isotropic incompressible elastomer, with shear modulus $\mu_{m}$, filled with a dilute distribution of rigid spherical particles that are bonded through isotropic incompressible interphases with shear modulus $\mu_{i}$ and thickness $t$. Three key points are worth remarking: 
- The dependence of the effective shear modulus (16) on $t$ enters through the interphase-thickness-toparticle-radius ratio $t / r$, or equivalently, through the ratio of concentration of interphases to concentration of particles $c_{i} / c_{p}$. A corollary of such a dimensionless dependence is that the result (16) applies not only to microstructures with monodisperse particles, but also to microstructures with polydisperse particles provided that all particles and surrounding interphases have the same interphase-thicknessto-particle-radius ratio $t / r$.

- The result (16) constitutes a generalization of the classical result of Einstein-Smallwood (Smallwood, 1944) for the effective shear modulus of a dilute suspension of rigid spherical particles in rubber with perfect bonding (i.e., without interphases) between the particles and the rubber. Indeed, in the absence of interphases when $c_{i}=0$, the effective shear modulus (16) reduces identically to the wellknown formula $\bar{\mu}^{\text {dil }}=\mu_{m}+5 / 2 \mu_{m} c_{p}$. The choices $\mu_{i}=\mu_{m}$ and $\mu_{i}=+\infty$ also recover this classical result; in the latter, $\bar{\mu}^{\text {dil }}=\mu_{m}+5 / 2 \mu_{m}\left(c_{p}+c_{i}\right)$ since the total concentration of particles is $c_{p}+c_{i}$ in that limiting case.

- A further salient feature of the solution (15)-(18) is that the stress fields inside the particles are not uniform; see Appendix A for details. Uniform intra-particle (stress and strain) fields are the hallmark of the classical solution of Einstein-Smallwood, and, more generally, that of Eshelby (1957), where no interphases are accounted for. The solution (15)-(18) reveals that the presence of interphases, however small, disrupts the uniformity of the fields inside the particles. The theoretical and practical implications of this feature are far reaching since many homogenization techniques (e.g., the MoriTanaka approximation and most techniques based on Hashin-Shtrikman-type variational principles) make critical use of the very fact that the fields in at least one of the underlying constituents are uniform. The employment of such techniques to study the behavior of particulate composites with interphases might hence lead to inaccurate results.

\subsection{An approximate closed-form solution for arbitrarily large deformations}

For arbitrarily large applied deformations $\overline{\mathbf{F}}$, the single-particle problem does not appear to admit an exact analytical solution. In the following two subsections, guided by earlier results for dilute suspensions of rigid particles without interphases (Lopez-Pamies, et al. 2013a), we first construct a FE solution for the effective stored-energy function $\bar{\Psi}$ from which we are then able to devise a closed-form approximation for it.

\subsubsection{Finite-element solution}

By virtue of the invariance of the equations of elastostatics under the transformation $(\mathbf{X}, \mathbf{x}) \rightarrow(\beta \mathbf{X}, \beta \mathbf{x})$, it is indifferent to consider the problem of an infinitely large elastomeric matrix containing a finite-size particle or that of a finite-size block of elastomer that contains a particle of infinitesimal size. In constructing a FE solution, we are compelled to consider the latter. Without loss of generality, we take the elastomer block to be a cube of side $L$. In this context, given that the radius $r$ of the particle and the thickness $t$ of its surrounding interphase must be necessarily finite, we need to identify how small their concentrations $c_{p}=4 \pi r^{3} / 3 L^{3}$ and $c_{i}=4 \pi\left[(r+t)^{3}-r^{3}\right] / 3 L^{3}$ ought to be in order to accurately approximate an infinitesimally small particle and infinitesimally small interphase. To this end, we carried out a parametric study with decreasing concentrations of the particle $c_{p}$ and interphase $c_{i}$. The results indicate that for combined values $c_{p}+c_{i} \leq 10^{-8}$, the particle and interphase behave effectively as infinitesimally small. Based on this analysis, all the calculations that follow are such that

$$
c_{p}+c_{i}=\frac{125 \pi}{48} \times 10^{-9}
$$

corresponding to a combined particle-interphase length of $r+t=1$ in a cube of side $L=800$.

The geometric and constitutive symmetries of the problem allow to perform the calculations in just one octant of the cube. A standard mesh generator is utilized to construct the 3D geometry for such an octant. The particle need not be meshed, instead, the nodes at the particle/interphase boundary are fixed in the undeformed configuration in order to model the rigid behavior of the particle. For illustration purposes, Fig. 


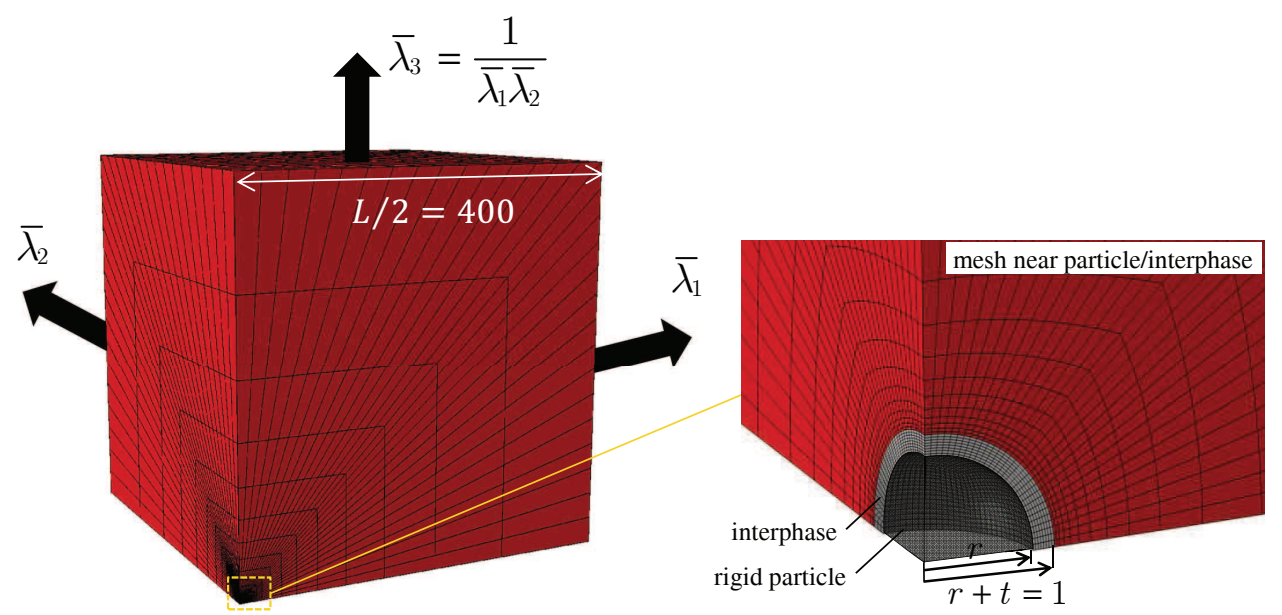

Figure 3: A representative finite element discretization — in the undeformed configuration — of a small rigid spherical particle of radius $r$ bonded to the matrix through an interphase of thickness $t=1-r$ located at the center of a cubic block with edge length $L=800$. The boundary of the cube is subjected to the stretches $\bar{\lambda}_{1}, \bar{\lambda}_{2}$, and $\bar{\lambda}_{3}=\left(\bar{\lambda}_{1} \bar{\lambda}_{2}\right)^{-1}$ aligned with the three principal axes of the cube.

3 shows the mesh utilized for the case with $r=5 / 6$ and $t=0.2 r=1 / 6$, which correspond to $c_{p} \approx 4.73 \times 10^{-9}$ and $c_{i} \approx 3.45 \times 10^{-9}$. Small elements are placed near the rigid particle at uniform angular intervals of $3^{\circ}$, while the radial length is gradually increased toward the outer boundary. The mesh consists of 18,900 brick elements with 675 elements on a radial plane and 28 layers along the radial direction. The interphase is comprised of 8 layers of elements in the radial direction. This discretization was selected after various mesh refinements were tried to assess sufficient mesh convergence. In selecting an appropriate type of element, we tested 8-node linear and 20-node quadratic hybrid elements, where the pressure is treated as a further degree of freedom in order to be able to handle the incompressibility of the Gaussian matrix and interphase. The 20-node quadratic elements with linearly varying pressure proved to have a faster convergence and thus were selected.

Given the overall isotropy and incompressibility of the problem, it suffices to restrict attention to affine boundary conditions (7) with deformation gradients of the diagonal form

$$
\overline{\mathbf{F}}=\operatorname{diag}\left(\bar{\lambda}_{1}, \bar{\lambda}_{2}, \bar{\lambda}_{3}=\frac{1}{\bar{\lambda}_{1} \bar{\lambda}_{2}}\right)
$$

We find it convenient to implement this type of loading conditions by following radial straining paths in principal-logarithmic-strain space. Specifically, we set

$$
\bar{\lambda}_{1}=\lambda \text { and } \bar{\lambda}_{2}=\lambda^{a}
$$

(and hence $\left.\bar{\lambda}_{3}=\left(\bar{\lambda}_{1} \bar{\lambda}_{2}\right)^{-1}=\lambda^{-(1+a)}\right)$, where $\lambda$ is a positive load parameter that takes the value of 1 in the undeformed configuration and $a \in \mathbb{R}$. Any desired macroscopic deformation state $\left(\bar{\lambda}_{1}, \bar{\lambda}_{2}, \bar{\lambda}_{3}=\left(\bar{\lambda}_{1} \bar{\lambda}_{2}\right)^{-1}\right)$ can be accessed by marching along (starting at $\lambda=1$ ) radial paths (21) with appropriate fixed values of the parameter $a$.

Under boundary conditions (7) with (20), the total elastic energy per unit undeformed volume computed from the FE model, denoted here by $\bar{\Psi}^{F E}$, turns out to be of the expected asymptotic form

$$
\bar{\Psi}^{F E}\left(\bar{\lambda}_{1}, \bar{\lambda}_{2}, c_{p}, c_{i}\right)=\frac{\mu_{m}}{2}\left[\bar{\lambda}_{1}^{2}+\bar{\lambda}_{2}^{2}+\frac{1}{\bar{\lambda}_{1}^{2} \bar{\lambda}_{2}^{2}}-3\right]+\mu_{m} H\left(\bar{\lambda}_{1}, \bar{\lambda}_{2}\right) c_{p}+\mu_{m} G\left(\bar{\lambda}_{1}, \bar{\lambda}_{2}\right) c_{i}
$$


to leading order in $c_{p}$ and $c_{i}$. In this expression, $H$ and $G$ are functions of the applied stretches $\bar{\lambda}_{1}$ and $\bar{\lambda}_{2}$ such that

$$
\begin{aligned}
& H\left(\bar{\lambda}_{1}, \bar{\lambda}_{2}\right)=H\left(\bar{\lambda}_{2}, \bar{\lambda}_{1}\right)=H\left(\bar{\lambda}_{1},\left(\bar{\lambda}_{1} \bar{\lambda}_{2}\right)^{-1}\right)=H\left(\left(\bar{\lambda}_{1} \bar{\lambda}_{2}\right)^{-1}, \bar{\lambda}_{1}\right)=H\left(\bar{\lambda}_{2},\left(\bar{\lambda}_{1} \bar{\lambda}_{2}\right)^{-1}\right)=H\left(\left(\bar{\lambda}_{1} \bar{\lambda}_{2}\right)^{-1}, \bar{\lambda}_{2}\right), \\
& G\left(\bar{\lambda}_{1}, \bar{\lambda}_{2}\right)=G\left(\bar{\lambda}_{2}, \bar{\lambda}_{1}\right)=G\left(\bar{\lambda}_{1},\left(\bar{\lambda}_{1} \bar{\lambda}_{2}\right)^{-1}\right)=G\left(\left(\bar{\lambda}_{1} \bar{\lambda}_{2}\right)^{-1}, \bar{\lambda}_{1}\right)=G\left(\bar{\lambda}_{2},\left(\bar{\lambda}_{1} \bar{\lambda}_{2}\right)^{-1}\right)=G\left(\left(\bar{\lambda}_{1} \bar{\lambda}_{2}\right)^{-1}, \bar{\lambda}_{2}\right),
\end{aligned}
$$

as a result of the overall isotropy and incompressibility; in addition to the applied stretches, the function $G$ depends also on the ratio $\mu_{i} / \mu_{m}$ between the shear moduli of the Gaussian interphase and the matrix, as well as on the ratio $t / r$ between the thickness of the interphase and the radius of the particle, but such a dependence is not stated explicitly here for notational simplicity. Now, in order to extract the correcting functions $H$ and $G$ from the computed values of $\bar{\Psi}^{F E}$, an expedient strategy is first to compute $\bar{\Psi}^{F E}$ in the absence of the interphase when $c_{i}=0$ so that

$$
H\left(\bar{\lambda}_{1}, \bar{\lambda}_{2}\right)=\frac{1}{c_{p}}\left\{\frac{1}{\mu_{m}} \bar{\Psi}^{F E}\left(\bar{\lambda}_{1}, \bar{\lambda}_{2}, c_{p}, 0\right)-\frac{1}{2}\left[\bar{\lambda}_{1}^{2}+\bar{\lambda}_{2}^{2}+\frac{1}{\bar{\lambda}_{1}^{2} \bar{\lambda}_{2}^{2}}-3\right]\right\} .
$$

Having determined $H$ from (24), we can readily extract the function $G$ from the computed values of $\bar{\Psi}^{F E}$ for the case when the interphase is accounted for, namely,

$$
G\left(\bar{\lambda}_{1}, \bar{\lambda}_{2}\right)=\frac{1}{c_{i}}\left\{\frac{1}{\mu_{m}} \bar{\Psi}^{F E}\left(\bar{\lambda}_{1}, \bar{\lambda}_{2}, c_{p}, c_{i}\right)-\frac{1}{2}\left[\bar{\lambda}_{1}^{2}+\bar{\lambda}_{2}^{2}+\frac{1}{\bar{\lambda}_{1}^{2} \bar{\lambda}_{2}^{2}}-3\right]-H\left(\bar{\lambda}_{1}, \bar{\lambda}_{2}\right) c_{p}\right\} .
$$

Here, it is important to emphasize that the correction terms in (22) are in the order of $10^{-9}$ or smaller, as dictated by the combined concentration of particle and interphase (19), and hence that the computation of $\bar{\Psi}^{F E}$ must be carefully carried out in double precision in order to be able to accurately determine the correcting functions $H$ and $G$ from (24) and (25). It is also important to emphasize that in the computation of the functions $H$ and $G$, by virtue of their symmetries (23), it suffices to restrict attention to radial loadings (21) with $\lambda \geq 1$ and $a \in[-0.5,1]$.

Results and discussion. Figure 4 shows the FE solution for the correcting function $H$. Part (a) of the figure shows the full 3D view of the function over a large range of stretches $\bar{\lambda}_{1}$ and $\bar{\lambda}_{2}$, while part (b) shows its $2 \mathrm{D}$ view along the axisymmetric shear loading with $\bar{\lambda}_{1}=\bar{\lambda}_{2}=\bar{\lambda}$.

Similarly, Fig. 5 shows the FE solution for the correcting function $G$. Part (a) of the figure shows the full $3 \mathrm{D}$ view of the function over a large range of stretches $\bar{\lambda}_{1}$ and $\bar{\lambda}_{2}$, while part (b) shows its 2D view along the axisymmetric shear loading with $\bar{\lambda}_{1}=\bar{\lambda}_{2}=\bar{\lambda}$. As opposed to $H$, the function $G$ does depend on the ratio $\mu_{i} / \mu_{m}$ between the shear moduli of the interphase and the matrix, as well as on the ratio $t / r$ between the thickness of the interphase and the radius of the particle. The results displayed in Fig. 5 correspond to the values $\mu_{i} / \mu_{m}=10$ and $t / r=0.1$. 


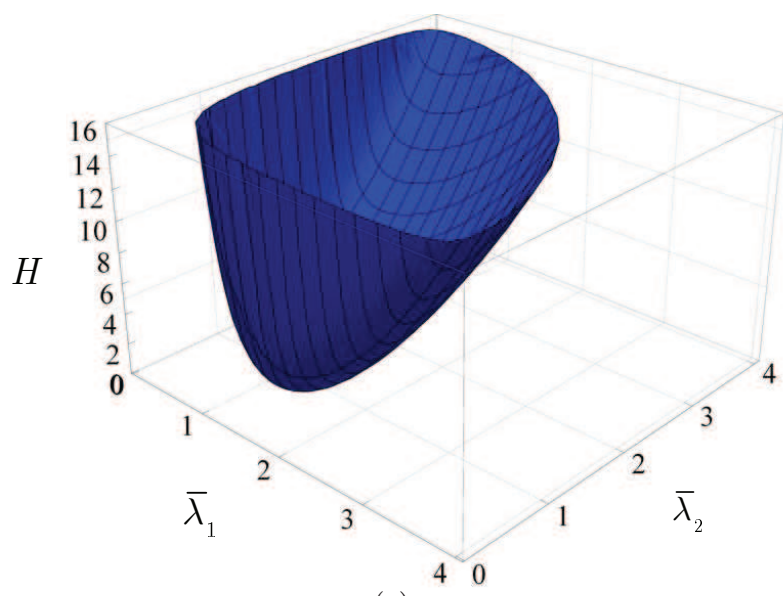

(a)

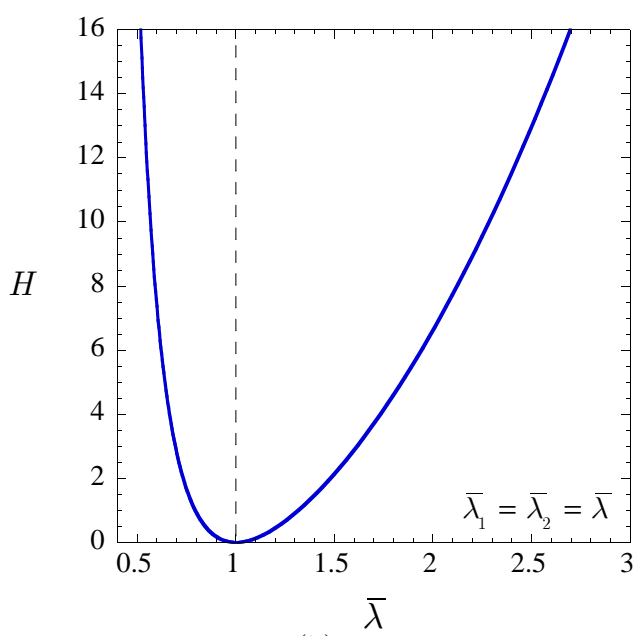

(b)

Figure 4: (a) Full 3D view of the FE solution for the correcting function $H$, defined in (22), over a large range of applied macroscopic stretches $\bar{\lambda}_{1}$ and $\bar{\lambda}_{2}$. (b) $2 \mathrm{D}$ view along the axisymmetric shear loading with $\bar{\lambda}_{1}=\bar{\lambda}_{2}=\bar{\lambda}$.

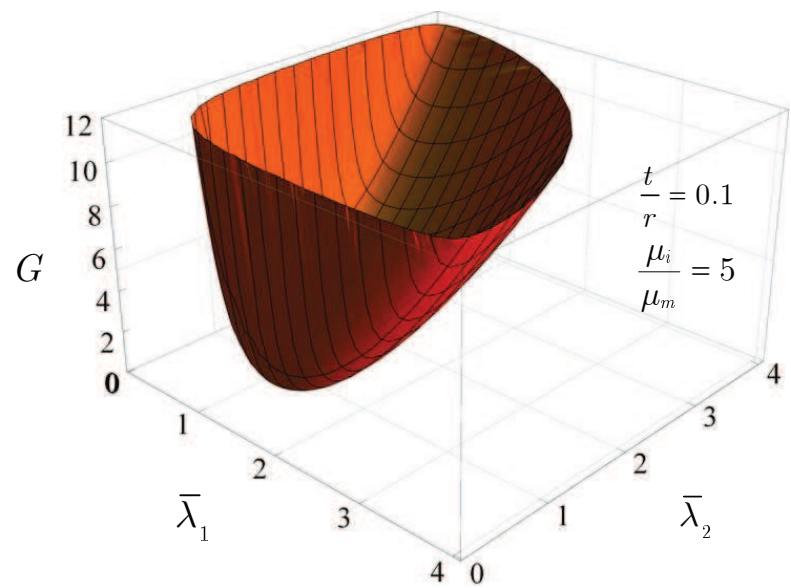

(a)

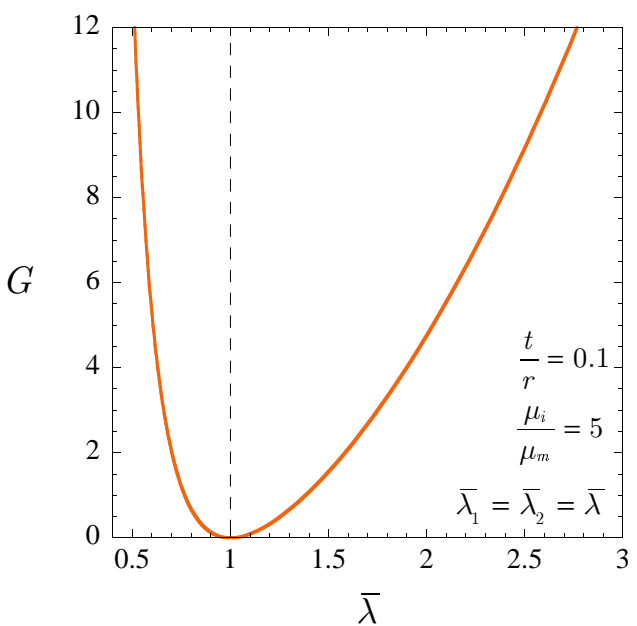

(b)

Figure 5: (a) Full 3D view of the FE solution for the correcting function $G$, defined in (22), over a large range of applied macroscopic stretches $\bar{\lambda}_{1}$ and $\bar{\lambda}_{2}$. (b) $2 \mathrm{D}$ view along the axisymmetric shear loading with $\bar{\lambda}_{1}=\bar{\lambda}_{2}=\bar{\lambda}_{\text {. The }}$ results correspond to an interphase that is five times stiffer than the matrix, $\mu_{i} / \mu_{m}=5$, whose thickness is one tenth the particle radius, $t / r=0.1$.

The dependence of $G$ on $\mu_{i} / \mu_{m}$ and $t / r$ is illustrated in Fig. 6. For purposes of visualization, the results are presented only for the case of axisymmetric shear loading when $\bar{\lambda}_{1}=\bar{\lambda}_{2}=\bar{\lambda}$. Specifically, Fig. 6 (a) displays the function $G$ for $\mu_{i} / \mu_{m}=2,5,10,20,+\infty$ and $t / r=0.1$. On the other hand, Fig. 6(b) displays $G$ for $t / r=0.05,0.1,0.2$ and $\mu_{i} / \mu_{m}=5$. An immediate observation from these plots is that $G$ is a monotonically increasing function of the interphase stiffness $\mu_{i} / \mu_{m}$, but a decreasing function of its thickness $t / r$. With respect to the dependence on the interphase stiffness, it is worth remarking that $G$ saturates quickly with increasing values of $\mu_{i} / \mu_{m}$. There is indeed little difference between the result for $\mu_{i} / \mu_{m}=10$ and that for $\mu_{i} / \mu_{m}=+\infty$. It is also worth remarking that $G=H$ for $\mu_{i} / \mu_{m}=+\infty$, as expected, since in this limiting case there is actually no interphase but instead a rigid spherical particle of radius $r+t$ perfectly bonded to the matrix. 


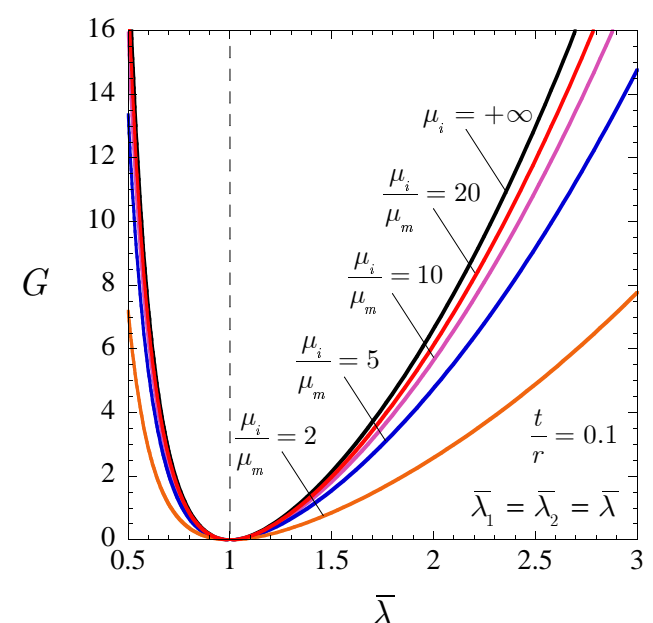

(a)

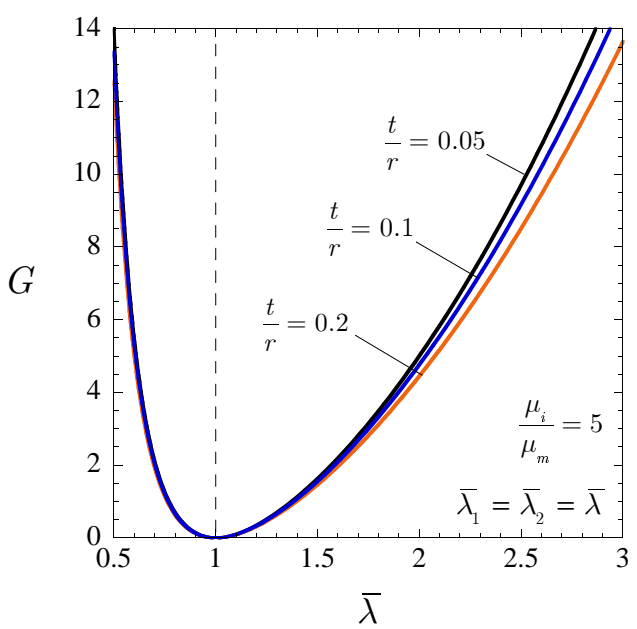

(b)

Figure 6: FE solutions for the correcting function $G$ along the axisymmetric shear loading with $\bar{\lambda}_{1}=\bar{\lambda}_{2}=\bar{\lambda}$. Part (a) shows results for various ratios $\mu_{i} / \mu_{m}$ between the interphase and matrix shear moduli at fixed $t / r=0.1$, while part (b) shows results for various ratios $t / r$ between the interphase thickness and particle radius at fixed $\mu_{i} / \mu_{m}=5$.

For further scrutiny of the correcting functions $H$ and $G$, Fig. 7 shows results for $H$ and $G$ in terms of the first and second principal invariants of the applied macroscopic loading: $\bar{I}_{1}=\overline{\mathbf{F}} \cdot \overline{\mathbf{F}}=\bar{\lambda}_{1}^{2}+\bar{\lambda}_{2}^{2}+\bar{\lambda}_{1}^{-2} \bar{\lambda}_{2}^{-2}$ and $\bar{I}_{2}=\overline{\mathbf{F}}^{-T} \cdot \overline{\mathbf{F}}^{-T}=\bar{\lambda}_{1}^{-2}+\bar{\lambda}_{2}^{-2}+\bar{\lambda}_{1}^{2} \bar{\lambda}_{2}^{2}$. Parts (a) and (c) show $H$ and $G$ as functions of $\bar{I}_{1}$ for the two fixed values $\bar{I}_{2}=4$ and 6 . Parts (b) and (d), on the other hand, show $H$ and $G$ as functions of $\bar{I}_{2}$ for the four fixed values $\bar{I}_{1}=4,7,10,13$. The results for $G$ correspond to the case of an interphase with $\mu_{i} / \mu_{m}=5$ and $t / r=0.1$. In the context of these plots, it is appropriate to recall that the constraint of incompressibility imposes a restriction on the physically allowable values of $\bar{I}_{1}$ and $\bar{I}_{2}$. Thus, for fixed $\bar{I}_{2}=4$ and 6 , the first invariant is restricted to take values in the ranges $\bar{I}_{1} \in[3.71,4.52]$ and $\bar{I}_{1} \in[4.72,9.34]$, respectively. For fixed $\bar{I}_{1}=4,7,10,13$, the allowable values of the second principal invariant are such that $\bar{I}_{2} \in[3.71,4.52]$, $\bar{I}_{2} \in[5.14,12.54], \bar{I}_{2} \in[6.22,25.20]$, and $\bar{I}_{2} \in[7.13,42.40]$, respectively. These are the ranges of values utilized in the figure.

The dominant observation from Fig. 7 is that both correcting functions $H$ and $G$ are approximately linear in $\bar{I}_{1}$ and independent of $\bar{I}_{2}$; while the results for $G$ in this figure correspond to the particular case of interphase stiffness $\mu_{i} / \mu_{m}=5$ and thickness $t / r=0.1$, the approximately linear dependence on $\bar{I}_{1}$ and independence from $\bar{I}_{2}$ of this function has been checked (through a parametric study) to be insensitive to the choice of values for $\mu_{i} / \mu_{m}$ and $t / r$. The fact that these macroscopic or average correcting functions are, in essence, functionally identical - namely, linear in $\bar{I}_{1}$ and independent of $\bar{I}_{2}$ - to the local storedenergy functions for the underlying Gaussian matrix and interphase is admittedly remarkable. Indeed, the functional character of the average behavior of nonlinear material systems is in general substantially different from that of its constituents, but that is not the case here. This is a most distinctive trait that we exploit next to generate a simple yet accurate closed-form approximation. 


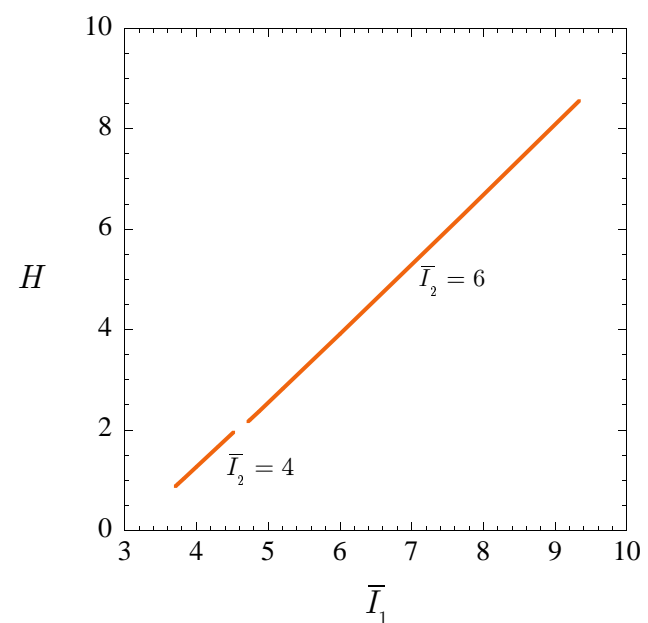

(a)

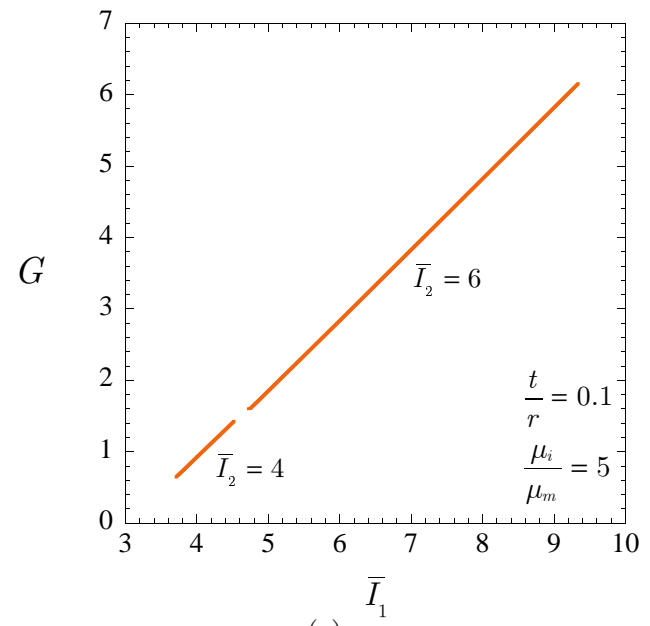

(c)

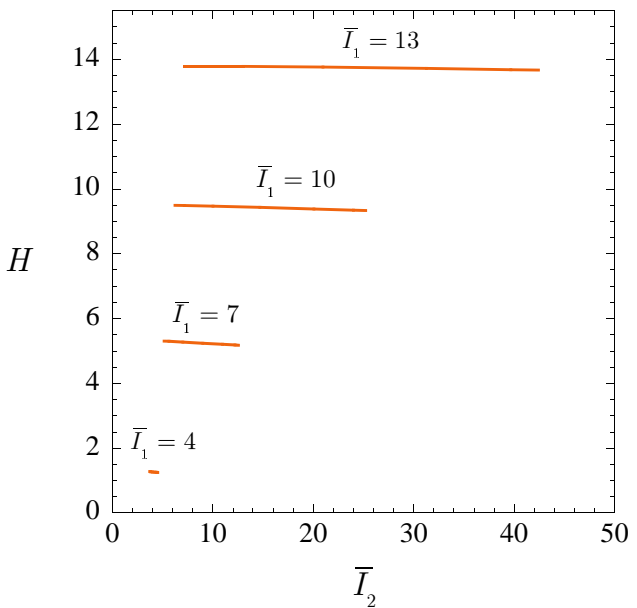

(b)

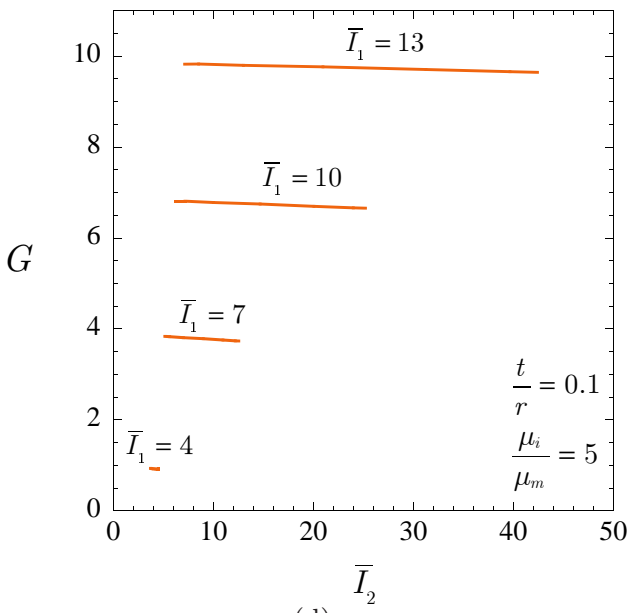

(d)

Figure 7: FE solutions for the correcting functions $H$ and $G$ plotted in terms of the principal invariants $\bar{I}_{1}=$ $\bar{\lambda}_{1}^{2}+\bar{\lambda}_{2}^{2}+\bar{\lambda}_{1}^{-2} \bar{\lambda}_{2}^{-2}$ and $\bar{I}_{2}=\bar{\lambda}_{1}^{-2}+\bar{\lambda}_{2}^{-2}+\bar{\lambda}_{1}^{2} \bar{\lambda}_{2}^{2}$. Parts (a) and (c) show $H$ and $G$ as functions of $\bar{I}_{1}$ for different fixed values of $\bar{I}_{2}$, whereas parts (b) and (d) show corresponding plots as functions of $\bar{I}_{2}$ for different fixed values of $\bar{I}_{1}$. The results shown for the function $G$ in (c) and (d) correspond to an interphase that is five times stiffer than the matrix, $\mu_{i} / \mu_{m}=5$, whose thickness is one tenth the particle radius, $t / r=0.1$.

\subsubsection{The proposed approximate closed-form solution}

In view of the analytical asymptotic solution (15) in the small-deformation limit together with the foregoing numerical observations for finite deformations, we propose the following closed-form approximations

$$
\begin{aligned}
& H\left(\bar{\lambda}_{1}, \bar{\lambda}_{2}\right)=\frac{5}{4}\left[\bar{\lambda}_{1}^{2}+\bar{\lambda}_{2}^{2}+\frac{1}{\bar{\lambda}_{1}^{2} \bar{\lambda}_{2}^{2}}-3\right] \\
& G\left(\bar{\lambda}_{1}, \bar{\lambda}_{2}\right)=\frac{5\left(\mu_{i}-\mu_{m}\right)\left(q_{1} \mu_{i}+q_{4} \mu_{m}\right)}{4\left(q_{1} \mu_{i}^{2}+q_{2} \mu_{i} \mu_{m}+q_{3} \mu_{m}^{2}\right)}\left[\bar{\lambda}_{1}^{2}+\bar{\lambda}_{2}^{2}+\frac{1}{\bar{\lambda}_{1}^{2} \bar{\lambda}_{2}^{2}}-3\right]
\end{aligned}
$$

for the correcting functions $H$ and $G$. By construction, the approximations (26)-(27) have the merit to be exact in the limit of small deformations, as $\bar{\lambda}_{1}, \bar{\lambda}_{2} \rightarrow 1$. For arbitrarily large deformations, although not exact, the approximations (26)-(27) are practically identical to the exact solution in a functional sense: 
they are linear in $\bar{I}_{1}\left(=\bar{\lambda}_{1}^{2}+\bar{\lambda}_{2}^{2}+\bar{\lambda}_{1}^{-2} \bar{\lambda}_{2}^{-2}\right)$ and independent of $\bar{I}_{2}\left(=\bar{\lambda}_{1}^{-2}+\bar{\lambda}_{2}^{-2}+\bar{\lambda}_{1}^{2} \bar{\lambda}_{2}^{2}\right)$. In addition, as illustrated by Figs. 8 and 9, the simple closed-form expressions (26)-(27) are remarkably accurate when compared with the corresponding FE solutions. Specifically, Fig. 8 shows comparisons between the proposed approximation (26) for the function $H$ and its FE solution. Similarly, Fig. 9 shows comparisons between the proposed approximation (27) for $G$ and its FE solution. Parts (a) and (b) of Fig. 9 correspond to the case of an interphase that is five times stiffer than the matrix, $\mu_{i} / \mu_{m}=5$, whose thickness is one tenth the particle radius, $t / r=0.1$, while parts (c) and (d) illustrate comparisons for various values of the ratios $\mu_{i} / \mu_{m}$ and $t / r$.

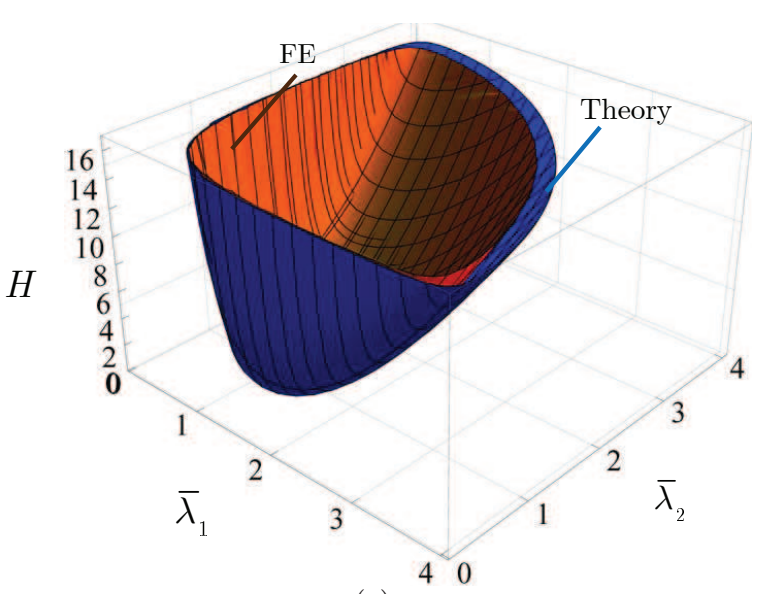

(a)

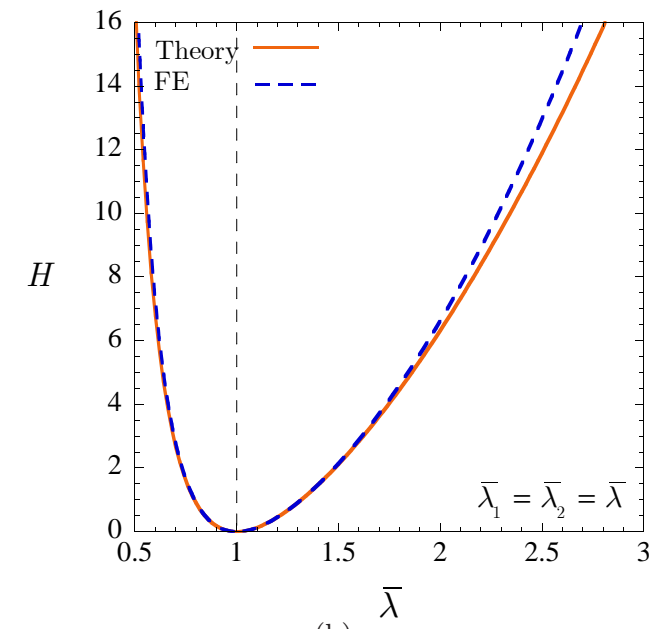

(b)

Figure 8: Comparison between the correcting function $H$ computed from the FE simulations and its closed-form approximation (26). Part (a) shows the comparison for a wide range of stretches $\bar{\lambda}_{1}$ and $\bar{\lambda}_{2}$, while part (b) shows the comparison for the case of axisymmetric deformations with $\bar{\lambda}_{1}=\bar{\lambda}_{2}=\bar{\lambda}$.

Making use of the approximations (26)-(27) for $H$ and $G$, we readily obtain the resulting closed-form approximate solution for the effective stored-energy function $\bar{\Psi}$ of a Gaussian elastomer with shear modulus $\mu_{m}$, filled with a dilute distribution of rigid spherical particles that are bonded through interphases of constant thickness $t$, made up of a different Gaussian elastomer with shear modulus $\mu_{i}$. The result reads as

$$
\begin{aligned}
\bar{\Psi}\left(\bar{I}_{1}, \bar{I}_{2}, c_{p}, c_{i}\right) & =\frac{\mu_{m}}{2}\left[\bar{I}_{1}-3\right]+\frac{5 \mu_{m}}{4}\left[\bar{I}_{1}-3\right] c_{p}+\frac{5\left(\mu_{i}-\mu_{m}\right)\left(q_{1} \mu_{i}+q_{4} \mu_{m}\right) \mu_{m}}{4\left(q_{1} \mu_{i}^{2}+q_{2} \mu_{i} \mu_{m}+q_{3} \mu_{m}^{2}\right)}\left[\bar{I}_{1}-3\right] c_{i} \\
& =\frac{\bar{\mu}^{\text {dil }}}{2}\left[\bar{I}_{1}-3\right]
\end{aligned}
$$

to leading order in the concentration of particles $c_{p}$ and interphases $c_{i}$. Here, it is recalled that the coefficients $q_{1}, q_{2}, q_{3}, q_{4}$ are given in terms of the concentration ratio $c_{i} / c_{p}$ by expressions (17), whereas the effective shear modulus $\bar{\mu}^{\text {dil }}$ is given by expression (16). Because of the above-discussed properties of the functions (26)(27), the approximate solution (28) is identical to the exact solution (15) in the limit of small deformations and, while not exact, qualitatively and quantitatively very close to the FE solution for arbitrarily large deformations. We conclude by remarking that the dependence of the effective stored-energy function (28) on $t$ enters via the effective shear modulus $\bar{\mu}^{\text {dil }}$ through the dimensionless interphase-thickness-to-particleradius ratio $t / r=\left(1+c_{i} / c_{p}\right)^{1 / 3}-1$. This implies that the result (28) applies not only to microstructures with monodisperse particles, but also to microstructures with polydisperse particles wherein all particles and surrounding interphases have the same aforementioned interphase-thickness-to-particle-radius ratio. 


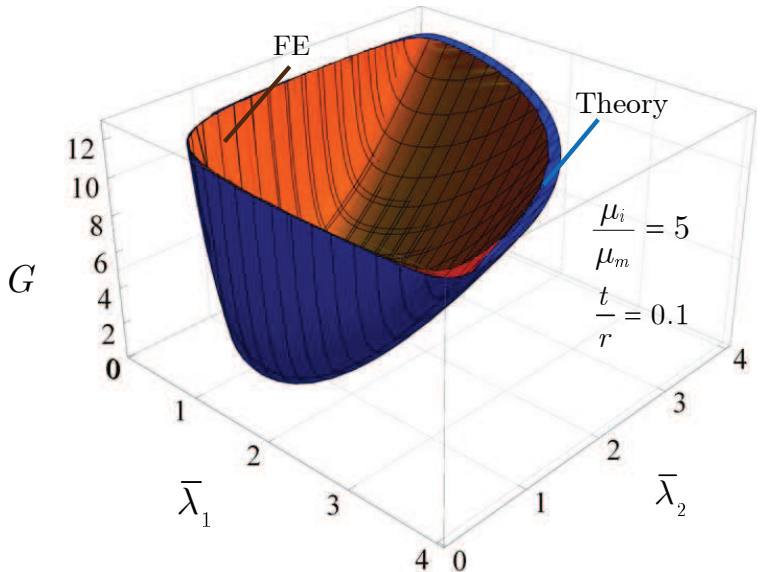

(a)

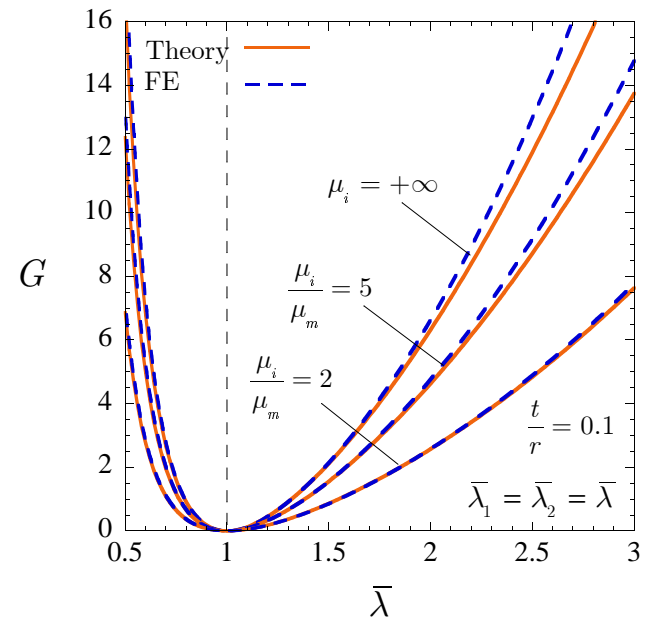

(c)

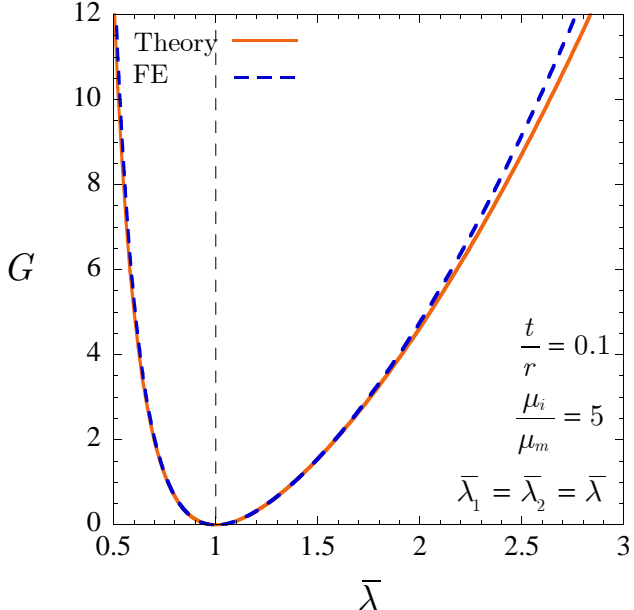

(b)

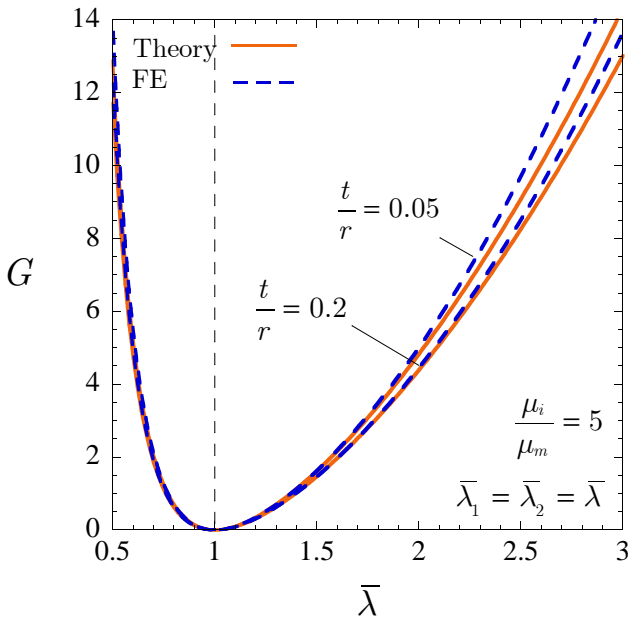

(d)

Figure 9: Comparison between the correcting function $G$ computed from the FE simulations and its closed-form approximation (27). Parts (a) and (b) show the comparison for a wide range of stretches $\bar{\lambda}_{1}$ and $\bar{\lambda}_{2}$ for $\mu_{i} / \mu_{m}=5$ and $t / r=0.1$. Part (c) shows results for various ratios $\mu_{i} / \mu_{m}$ between the interphase and the matrix shear moduli at fixed $t / r=0.1$, while part (d) shows results for various ratios $t / r$ between the interphase thickness and particle radius at fixed $\mu_{i} / \mu_{m}=5$ along axisymmetric deformations with $\bar{\lambda}_{1}=\bar{\lambda}_{2}=\bar{\lambda}$.

\section{Finite concentration of particles and interphases in non-Gaussian elastomers}

Next, we construct a solution for the effective stored-energy function $\bar{\Psi}$, as defined by (13) with (9), of non-Gaussian elastomers filled with an isotropic distribution of rigid spherical particles of polydisperse sizes and finite concentration $c_{p}$ that are bonded through constant-thickness Gaussian interphases of finite concentration $c_{i}$. This is accomplished by making use of two different techniques in two successive steps. First, as elaborated in Section 4.1, the fundamental dilute solution (28) is utilized within the context of an iterated homogenization method in finite elasticity (Lopez-Pamies, 2010b; 2014) to generate a finiteconcentration solution for filled Gaussian elastomers. In Section 4.2, this finite-concentration result is then employed within the context of a variational nonlinear comparison medium method (Lopez-Pamies et al., $2013 \mathrm{~b}$ ) to generate in turn a corresponding solution for the more general case when the underlying elastomeric matrix is non-Gaussian, as characterized by any $I_{1}$-based stored-energy function $\Psi_{m}\left(I_{1}\right)$ of choice. 


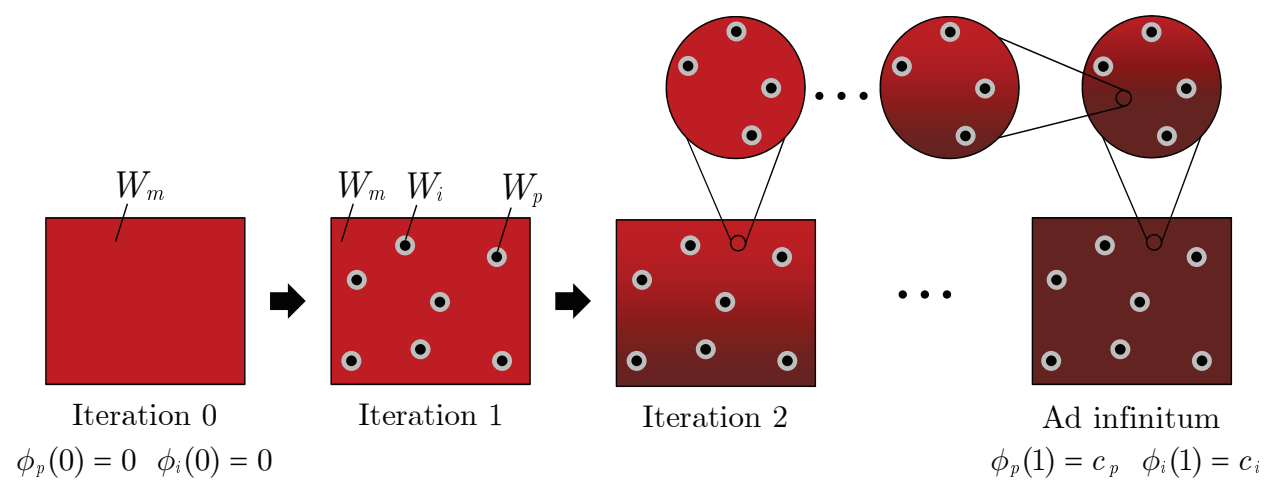

Figure 10: Schematic of the iterative dilute construction process of an elastomer (characterized by a stored-energy function $W_{m}$ ) filled with particles (characterized by a stored-energy function $W_{p}$ ) at finite concentration $c_{p}$ bonded through interphases (characterized by a stored-energy function $W_{i}$ ) at finite concentration $c_{i}$.

Iterated dilute homogenization methods are a class of iterative techniques that make use of results for the overall properties of dilute composites in order to generate corresponding results for composites with finite concentration of constituents. Within the non-convex realm of finite elasticity, extending the formulation of Lopez-Pamies (2010b) for two-phase composites, Lopez-Pamies (2014) has put forward an iterated dilute homogenization technique applicable to composite materials with any number of phases. For the generic three-phase problem outlined in Sections 2.1 and 2.2, assuming that the effective stored-energy function (9) is of the asymptotic form

$$
\bar{W}\left(\overline{\mathbf{F}}, c_{p}, c_{i}\right)=W_{m}(\overline{\mathbf{F}})+\mathcal{H}\left\{W_{m}, W_{p}, W_{i} ; \overline{\mathbf{F}}\right\} c_{p}+\mathcal{G}\left\{W_{m}, W_{p}, W_{i} ; \overline{\mathbf{F}}\right\} c_{i}
$$

to $O\left(c_{p}\right)$ and $O\left(c_{i}\right)$, the formulation states that the effective stored-energy function (9) of elastomers filled with particles bonded through interphases is given implicitly by the differential equation

$$
\begin{aligned}
\left(1-\phi_{p}-\phi_{i}\right) \frac{\partial \bar{W}}{\partial \tau}= & {\left[\left(1-\phi_{i}\right) \frac{\mathrm{d} \phi_{p}}{\mathrm{~d} \tau}+\phi_{p} \frac{\mathrm{d} \phi_{i}}{\mathrm{~d} \tau}\right] \mathcal{H}\left\{\bar{W}, W_{p}, W_{i} ; \overline{\mathbf{F}}\right\}+} \\
& {\left[\left(1-\phi_{p}\right) \frac{\mathrm{d} \phi_{i}}{\mathrm{~d} \tau}+\phi_{i} \frac{\mathrm{d} \phi_{p}}{\mathrm{~d} \tau}\right] \mathcal{G}\left\{\bar{W}, W_{p}, W_{i} ; \overline{\mathbf{F}}\right\} }
\end{aligned}
$$

subject to the initial condition

$$
\left.\bar{W}\left(\overline{\mathbf{F}}, c_{p}, c_{i}\right)\right|_{\tau=0}=W_{m}(\overline{\mathbf{F}}) .
$$

The functionals $\mathcal{H}$ and $\mathcal{G}$ in (30) are the same as in the asymptotic result (29), $\tau$ is a time-like variable taking values from 0 and 1 , the range over which the differential equation (30) must be integrated, and $\phi_{p}(\tau)$ and $\phi_{i}(\tau)$ are non-negative, non-decreasing functions of choice that must satisfy the properties $\phi_{p}(\tau)+\phi_{i}(\tau) \leq 1$, $\phi_{p}(0)=\phi_{i}(0)=0, \phi_{p}(1)=c_{p}, \phi_{i}(1)=c_{i}$. More specifically, the functions $\phi_{p}(\tau)$ and $\phi_{i}(\tau)$ characterize the manner in which the composite is constructed and thus contain microstructural information; see Fig. 10 for a schematic depiction of the iterative construction process. The interested reader is referred to Section 3.1 of Lopez-Pamies (2014) for the derivation and full description of the above results. Here, it suffices to remark that knowledge of an exact (approximate) dilute solution (29) allows to compute exact (approximate) non-dilute solutions via the initial-value problem (30)-(31). And that, by construction, such non-dilute solutions correspond to polydisperse microstructures with particles of infinitely many sizes. This feature is of practical relevance here because standard reinforcing fillers (e.g., carbon black and silica) typically agglomerate, resulting effectively in polydisperse microstructures with "particles" of many different sizes. 
When specialized to the class of isotropic incompressible filled elastomers described in Section 2.3, assuming that the matrix is Gaussian and employing the analytical approximation (28) as (the finite-branch of) the dilute solution (29), the formulation (30)-(31) generates the following result:

$$
\bar{\Psi}\left(\bar{I}_{1}, \bar{I}_{2}, c_{p}, c_{i}\right)=\frac{\bar{\mu}}{2}\left[\bar{I}_{1}-3\right]
$$

with the coefficient $\bar{\mu}$ being defined implicitly by the first-order nonlinear ode

$$
\left(1-\phi_{p}-\phi_{i}\right) \frac{\mathrm{d} \bar{\mu}}{\mathrm{d} \tau}=\left[\left(1-\phi_{i}\right) \frac{\mathrm{d} \phi_{p}}{\mathrm{~d} \tau}+\phi_{p} \frac{\mathrm{d} \phi_{i}}{\mathrm{~d} \tau}\right] \frac{5 \bar{\mu}}{2}+\left[\left(1-\phi_{p}\right) \frac{\mathrm{d} \phi_{i}}{\mathrm{~d} \tau}+\phi_{i} \frac{\mathrm{d} \phi_{p}}{\mathrm{~d} \tau}\right] \frac{5 \bar{\mu}\left(\mu_{i}-\bar{\mu}\right)\left(\widehat{q}_{1} \mu_{i}+\widehat{q}_{4} \bar{\mu}\right)}{2\left(\widehat{q}_{1} \mu_{i}^{2}+\widehat{q}_{2} \mu_{i} \bar{\mu}+\widehat{q}_{3} \bar{\mu}^{2}\right)},
$$

again, to be integrated from $\tau=0$ to $\tau=1$, subject to the initial condition

$$
\left.\bar{\mu}\right|_{\tau=0}=\mu_{m},
$$

where

$$
\begin{aligned}
& \widehat{q}_{1}=38 \widehat{k}^{10}+225 \widehat{k}^{7}-336 \widehat{k}^{5}+200 \widehat{k}^{3}+48, \\
& \widehat{q}_{2}=89 \widehat{k}^{10}+75 \widehat{k}^{7}-168 \widehat{k}^{5}+100 \widehat{k}^{3}-96, \\
& \widehat{q}_{3}=48 \widehat{k}^{10}-300 \widehat{k}^{7}+504 \widehat{k}^{5}-300 \widehat{k}^{3}+48 \\
& \widehat{q}_{4}=\frac{4(\widehat{k}-1)^{3}\left(2 \widehat{k}^{3}+3\right)\left(4 \widehat{k}^{6}+16 \widehat{k}^{5}+40 \widehat{k}^{4}+55 \widehat{k}^{3}+40 \widehat{k}^{2}+16 \widehat{k}+4\right)}{\widehat{k}^{2}+\widehat{k}+1}
\end{aligned}
$$

with

$$
\widehat{k}=\left(1+\frac{\left(1-\phi_{p}\right) \frac{\mathrm{d} \phi_{i}}{\mathrm{~d} \tau}+\phi_{i} \frac{\mathrm{d} \phi_{p}}{\mathrm{~d} \tau}}{\left(1-\phi_{i}\right) \frac{\mathrm{d} \phi_{p}}{\mathrm{~d} \tau}+\phi_{p} \frac{\mathrm{d} \phi_{i}}{\mathrm{~d} \tau}}\right)^{1 / 3} .
$$

Expression (32) with (33)-(36) corresponds to the effective stored-energy function of a Gaussian elastomer, with shear modulus $\mu_{m}$, filled with an isotropic distribution of polydisperse rigid spherical particles at finite concentration $c_{p}$ that are bonded through constant-thickness Gaussian interphases, with shear modulus $\mu_{i}$ and finite concentration $c_{i}$. Thorough comments on the theoretical and practical merits of this result are deferred to Section 4.2, where the more general case of filled elastomers with non-Gaussian matrix is addressed. At this stage, it is important to emphasize, however, that in addition to its explicit dependence on the concentration of particles $c_{p}$ and interphases $c_{i}$, the result (32) depends on the microstructure through the functions $\phi_{p}$ and $\phi_{i}$, which, again, characterize the way in which the composite is constructed.

Microstructures with constant interphase-thickness-to-particle-radius ratio. In this work, for relative simplicity, we shall consider microstructures wherein all filler particles, irrespectively of their size, are surrounded by interphases with the same interphase-thickness-to-particle-radius ratio. This amounts to choosing

$$
\phi_{p}=c_{p} \tau \quad \text { and } \quad \phi_{i}=c_{i} \tau
$$

in which case $\widehat{q}_{1}=q_{1}, \widehat{q}_{2}=q_{2}, \widehat{q}_{3}=q_{3}, \widehat{q}_{4}=q_{4}, \widehat{k}=k$, and the initial-value problem (33)-(34) for the coefficient $\bar{\mu}$ reduces to

$$
\left[1-\left(c_{p}+c_{i}\right) \tau\right] \frac{\mathrm{d} \bar{\mu}}{\mathrm{d} \tau}=\frac{5 c_{p}}{2} \bar{\mu}+\frac{5 c_{i}\left(\mu_{i}-\bar{\mu}\right)\left(q_{1} \mu_{i}+q_{4} \bar{\mu}\right)}{2\left(q_{1} \mu_{i}^{2}+q_{2} \mu_{i} \bar{\mu}+q_{3} \bar{\mu}^{2}\right)} \bar{\mu} \quad \text { with }\left.\quad \bar{\mu}\right|_{\tau=0}=\mu_{m},
$$

where, again, $q_{1}, q_{2}, q_{3}, q_{4}$, and $k$ are given explicitly in terms of the concentration ratio $c_{i} / c_{p}$ by expressions (17) and (18). Upon integration from $\tau=0$ to $\tau=1$, the ode (38) $)_{1}$ takes the form

$$
\int_{\mu_{m}}^{\bar{\mu}} \frac{\mathrm{d} z}{\frac{5 c_{p}}{2} z+\frac{5 c_{i}\left(\mu_{i}-z\right)\left(q_{1} \mu_{i}+q_{4} z\right)}{2\left(q_{1} \mu_{i}^{2}+q_{2} \mu_{i} z+q_{3} z^{2}\right)} z}=-\frac{\ln \left[1-c_{p}-c_{i}\right]}{c_{p}+c_{i}} .
$$


Further, upon recognizing that the integral in (39) can be carried out explicitly (together with some algebraic manipulation), this last equation can be rewritten as

$$
\begin{aligned}
\mathcal{F}_{1}\left\{\bar{\mu}, \mu_{m}\right\} \doteq & \frac{c_{i}\left[q_{4}^{2}-q_{4}\left(q_{1}+2 q_{2}+q_{3}\right)+q_{1} q_{3}\right]+c_{p}\left[q_{3}\left(2 q_{1}+q_{2}\right)-q_{4}\left(q_{2}+2 q_{3}\right)\right]}{\sqrt{c_{i}^{2}\left(q_{1}+q_{4}\right)^{2}+2 c_{i} c_{p}\left[q_{4}\left(2 q_{1}+q_{2}\right)-q_{1}\left(q_{2}+2 q_{3}\right)\right]+c_{p}^{2}\left(q_{2}^{2}-4 q_{1} q_{3}\right)}} \times \\
& \left\{\tanh ^{-1}\left[\frac{c_{i}\left(q_{4}-q_{1}\right)+c_{p} q_{2}+2\left(c_{p} q_{3}-c_{i} q_{4}\right) \frac{\bar{\mu}}{\mu_{i}}}{\sqrt{c_{i}^{2}\left(q_{1}+q_{4}\right)^{2}+2 c_{i} c_{p}\left[q_{4}\left(2 q_{1}+q_{2}\right)-q_{1}\left(q_{2}+2 q_{3}\right)\right]+c_{p}^{2}\left(q_{2}^{2}-4 q_{1} q_{3}\right)}}\right]-\right. \\
& \left.\tanh ^{-1}\left[\frac{\left.c_{1}\right)+c_{p} q_{2}+2\left(c_{p} q_{3}-c_{i} q_{4}\right) \frac{\mu_{m}}{\mu_{i}}}{\sqrt{c_{i}^{2}\left(q_{1}+q_{4}\right)^{2}+2 c_{i} c_{p}\left[q_{4}\left(2 q_{1}+q_{2}\right)-q_{1}\left(q_{2}+2 q_{3}\right)\right]+c_{p}^{2}\left(q_{2}^{2}-4 q_{1} q_{3}\right)}}\right]\right\}- \\
& \frac{1}{2}\left(q_{3}+q_{4}\right) \ln \left[\frac{c_{i}\left(\bar{\mu}-\mu_{i}\right)\left(\mu_{i} q_{1}+q_{4} \bar{\mu}\right)-c_{p}\left(\mu_{i}^{2} q_{1}+\mu_{i} q_{2} \bar{\mu}+q_{3} \bar{\mu}^{2}\right)}{c_{i}\left(\mu_{m}-\mu_{i}\right)\left(\mu_{i} q_{1}+q_{4} \mu_{m}\right)-c_{p}\left(\mu_{i}^{2} q_{1}+\mu_{i} q_{2} \mu_{m}+q_{3} \mu_{m}^{2}\right)}\right]+ \\
& \left(q_{4}-\frac{c_{p}}{c_{i}} q_{3}\right) \ln \left[\left(1-c_{i}-c_{p}\right)^{5 / 2} \frac{\bar{\mu}}{\mu_{m}}\right]=0,
\end{aligned}
$$

where the function $\mathcal{F}_{1}$ has been introduced for later reference. In general, as discussed in more detail below, equation (40) does not admit an explicit solution for the coefficient $\bar{\mu}$. For given values of $\mu_{m}, \mu_{i}, c_{p}$, and $c_{i}$ it is, however, straightforward to generate a numerical solution for it.

\subsection{Filled elastomers with non-Gaussian matrix}

Comparison medium methods are variational techniques that allow to generate approximations for the overall properties of composites in terms of the properties of "simpler" comparison media. Generalizing ideas from the works of Talbot and Willis (1985), Ponte Castañeda (1991), Willis (1994), and deBotton and Shmuel (2010), Lopez-Pamies et al. (2013b) have introduced a nonlinear comparison medium approach that is capable to deal with the general types of non-convex behaviors inherent to finite elasticity. For the problem of filled elastomers formulated in Sections 2.1 and 2.2, the method provides the following variational approximation for the effective stored-energy function (9):

$\bar{W}\left(\overline{\mathbf{F}}, c_{p}, c_{i}\right)=\left\{\begin{array}{l}\max _{W_{0}}\left\{\min _{\mathbf{F} \in \mathcal{K}} \frac{1}{|\Omega|} \int_{\Omega} W_{0}(\mathbf{X}, \mathbf{F}) \mathrm{d} \mathbf{X}+\frac{1}{|\Omega|} \int_{\Omega} \min _{\mathbf{A}, a}\left[f(\mathbf{X}, \mathbf{A}, a)-f_{0}(\mathbf{X}, \mathbf{A}, a)\right] \mathrm{d} \mathbf{X}\right\} \text { if } f-f_{0}>-\infty \\ \min _{W_{0}}\left\{\min _{\mathbf{F} \in \mathcal{K}} \frac{1}{|\Omega|} \int_{\Omega} W_{0}(\mathbf{X}, \mathbf{F}) \mathrm{d} \mathbf{X}+\frac{1}{|\Omega|} \int_{\Omega} \max _{\mathbf{A}, a}\left[f(\mathbf{X}, \mathbf{A}, a)-f_{0}(\mathbf{X}, \mathbf{A}, a)\right] \mathrm{d} \mathbf{X}\right\} \text { if } f-f_{0}<+\infty\end{array}\right.$.

In this expression, $W_{0}$ stands for the local stored-energy function of any comparison medium of choice, possibly heterogeneous, while the functions $f$ and $f_{0}$ are defined such that $f(\mathbf{X}, \mathbf{F}, J)=W(\mathbf{X}, \mathbf{F})$ and $f_{0}(\mathbf{X}, \mathbf{F}, J)=W_{0}(\mathbf{X}, \mathbf{F})$ when $J=\operatorname{det} \mathbf{F}$. The interested reader is referred to Section 4.1 of Lopez-Pamies et al. (2013b) for the derivation and full description of the above result. Here, it suffices to remark that knowledge of the overall nonlinear elastic response of a medium with local energy $W_{0}$, as characterized by its effective stored-energy function $\min _{\mathbf{F} \in \mathcal{K}}|\Omega|^{-1} \int_{\Omega} W_{0}(\mathbf{X}, \mathbf{F}) \mathrm{d} \mathbf{X}$, allows to compute a solution (approximate in general, but possibly exact in some cases) for the effective stored-energy function $\bar{W}$ of the filled elastomer of interest via the variational relation (41).

When specialized to the class of isotropic incompressible filled elastomers described in Section 2.3, by taking the comparison medium to be a filled Gaussian elastomer with the same microstructure and the same constitutive behaviors for the underlying rigid particles and Gaussian interphases as the actual filled elastomer of interest,

$$
W_{0}(\mathbf{X}, \mathbf{F})= \begin{cases}{\left[1-\theta_{p}(\mathbf{X})-\theta_{i}(\mathbf{X})\right] \frac{\mu_{0}}{2}\left[I_{1}-3\right]+\theta_{p}(\mathbf{X}) \Psi_{p}\left(I_{1}\right)+\theta_{i}(\mathbf{X}) \frac{\mu_{i}}{2}\left[I_{1}-3\right]} & \text { if } \quad J=1 \\ +\infty & \text { otherwise }\end{cases}
$$


with

$$
\Psi_{p}\left(I_{1}\right)= \begin{cases}0 & \text { if } I_{1}=3 \\ +\infty & \text { otherwise }\end{cases}
$$

and employing the approximation (32) as (the finite-branch of) the effective stored-energy function of such a comparison medium, the formulation (41) generates the following result:

$$
\bar{\Psi}\left(\bar{I}_{1}, \bar{I}_{2}, c_{p}, c_{i}\right)=\left\{\begin{array}{l}
\max _{\mu_{0}}\left\{\frac{\bar{\mu}_{0}}{2}\left[\bar{I}_{1}-3\right]+\left(1-c_{p}-c_{i}\right) \min _{\mathcal{I}_{1}}\left[\Psi_{m}\left(\mathcal{I}_{1}\right)-\frac{\mu_{0}}{2}\left[\mathcal{I}_{1}-3\right]\right] \text { if } \Psi_{m}\left(\mathcal{I}_{1}\right)-\mathcal{I}_{1}>-\infty\right. \\
\min _{\mu_{0}}\left\{\frac{\bar{\mu}_{0}}{2}\left[\bar{I}_{1}-3\right]+\left(1-c_{p}-c_{i}\right) \max _{\mathcal{I}_{1}}\left[\Psi_{m}\left(\mathcal{I}_{1}\right)-\frac{\mu_{0}}{2}\left[\mathcal{I}_{1}-3\right]\right] \text { if } \Psi_{m}\left(\mathcal{I}_{1}\right)-\mathcal{I}_{1}<+\infty\right.
\end{array},\right.
$$

where the coefficient $\bar{\mu}_{0}$ is defined implicitly by the nonlinear algebraic equation $\mathcal{F}_{1}\left\{\bar{\mu}_{0}, \mu_{0}\right\}=0$, cf. equation (40). In view of the monotonicity $(11)_{1}$ of the function $\Psi_{m}$, the max-min and min-max problems in (44) are solved by exactly the same stationary conditions

$$
\Psi_{m}^{\prime}\left(\mathcal{I}_{1}\right)=\frac{\mu_{0}}{2} \quad \text { and } \quad \frac{\mathrm{d} \bar{\mu}_{0}}{\mathrm{~d} \mu_{0}}\left[\bar{I}_{1}-3\right]-\left(1-c_{p}-c_{i}\right)\left[\mathcal{I}_{1}-3\right]=0,
$$

irrespectively of the growth conditions of $\Psi_{m}$. Making use of these relations, the effective stored-energy function (44) can be written more explicitly as

$$
\bar{\Psi}\left(\bar{I}_{1}, \bar{I}_{2}, c_{p}, c_{i}\right)=\left(1-c_{p}-c_{i}\right) \Psi_{m}\left(\mathcal{I}_{1}\right)+\frac{\bar{\mu}_{0}}{2}\left[\bar{I}_{1}-3\right]-\left(1-c_{p}-c_{i}\right) \frac{\mu_{0}}{2}\left[\mathcal{I}_{1}-3\right]
$$

with

$$
\mathcal{I}_{1}=\left(\frac{c_{p} \bar{\mu}_{0}+\frac{\left(\mu_{i}-\bar{\mu}_{0}\right)\left(q_{1} \mu_{i}+q_{4} \bar{\mu}_{0}\right)}{q_{1} \mu_{i}^{2}+q_{2} \mu_{i} \bar{\mu}_{0}+q_{3} \bar{\mu}_{0}^{2}} c_{i} \bar{\mu}_{0}}{c_{p} \mu_{0}+\frac{\left(\mu_{i}-\mu_{0}\right)\left(q_{1} \mu_{i}+q_{4} \mu_{0}\right)}{q_{1} \mu_{i}^{2}+q_{2} \mu_{i} \mu_{0}+q_{3} \mu_{0}^{2}} c_{i} \mu_{0}}\right) \frac{\left[\bar{I}_{1}-3\right]}{1-c_{p}-c_{i}}+3
$$

and the variables $\bar{\mu}_{0}, \mu_{0}$ being defined implicitly by the system of two coupled nonlinear algebraic equations

$$
\mathcal{F}_{1}\left\{\bar{\mu}_{0}, \mu_{0}\right\}=0, \quad \mathcal{F}_{2}\left\{\bar{\mu}_{0}, \mu_{0}\right\} \doteq \Psi_{m}^{\prime}\left(\mathcal{I}_{1}\right)-\frac{\mu_{0}}{2}=0,
$$

where it is recalled that the coefficients $q_{1}, q_{2}, q_{3}, q_{4}$ are given explicitly in terms of the concentration ratio $c_{i} / c_{p}$ by expressions (17) and the function $\mathcal{F}_{1}$ is defined by expression (40).

The effective stored-energy function (46) with (47)-(48) constitutes the main result of this paper. It characterizes the overall nonlinear elastic response of a non-Gaussian elastomer, with stored-energy function $\Psi_{m}$, filled with an isotropic distribution of rigid spherical particles, of polydisperse sizes and finite concentration $c_{p}$, that are bonded to the elastomer through Gaussian interphases with shear modulus $\mu_{i}$, finite concentration $c_{i}$, and constant thickness-to-particle-radius ratio $\left(1+c_{i} / c_{p}\right)^{1 / 3}-1$. The following theoretical and practical remarks are in order:

$i$. In terms of the macroscopic first Piola-Kirchhoff stress tensor $\overline{\mathbf{S}}$ and macroscopic deformation gradient tensor $\overline{\mathbf{F}}$, the constitutive response implied by the effective stored-energy function (46) is given by

$$
\begin{aligned}
\overline{\mathbf{S}} & =2 \frac{\partial \bar{\Psi}}{\partial \bar{I}_{1}} \overline{\mathbf{F}}-\bar{p} \overline{\mathbf{F}}^{-T} \\
& =\bar{\mu}_{0} \overline{\mathbf{F}}-\bar{p} \overline{\mathbf{F}}^{-T}
\end{aligned}
$$

where $\bar{p}$ stands for the arbitrary hydrostatic pressure associated with the incompressibility constraint $\operatorname{det} \overline{\mathbf{F}}=1$ and, again, the coefficient $\bar{\mu}_{0}$ is defined implicitly by the system of two coupled nonlinear algebraic equations (48), which ultimately depend on the concentration of the particles $c_{p}$, the concentration of the interphases $c_{i}$, the stored-energy function of the matrix $\Psi_{m}$, the stiffness of the interphases $\mu_{i}$, and the applied loading via the first principal invariant $\bar{I}_{1}=\overline{\mathbf{F}} \cdot \overline{\mathbf{F}}$. 
ii. The effective stored-energy function (46) is independent of the second principal invariant $\bar{I}_{2}=\overline{\mathbf{F}}^{-T}$. $\overline{\mathbf{F}}^{-T}$. The origin of this independence can be traced back to the choice of approximation (28) for the dilute response of filled Gaussian elastomers, which neglects the weak but existent dependence on $\bar{I}_{2}$ of the exact solution in order to favor analytical tractability (see Section 3.2.1). Neither the iterated dilute homogenization procedure to account for finite concentrations of particles and interphases (Section 4.1), nor the comparison medium procedure to account for non-Gaussian behavior (Section 4.2) introduced dependence on $\bar{I}_{2}$ thereafter. This suggests that the response of any filled $I_{1}$-based non-Gaussian elastomer is by and large independent of $\bar{I}_{2}$. The FE simulations presented below provide further support that this is indeed the case.

iii. For stored-energy functions $\Psi_{m}$ that are convex in $I_{1}$,

$$
\Psi_{m}^{\prime}\left(I_{1}\right)>0 \quad \text { and } \quad \Psi_{m}^{\prime \prime}\left(I_{1}\right) \geq 0
$$

it follows that

$$
\begin{aligned}
& \frac{\partial \bar{\Psi}}{\partial \bar{I}_{1}}\left(\bar{I}_{1}, \bar{I}_{2}, c_{p}, c_{i}\right)>0 \\
& \frac{\partial \bar{\Psi}}{\partial \bar{I}_{1}}\left(\bar{I}_{1}, \bar{I}_{2}, c_{p}, c_{i}\right)+2\left[\bar{I}_{1}-\bar{\lambda}_{\alpha}^{2}-2 \bar{\lambda}_{\alpha}^{-1}\right] \frac{\partial^{2} \bar{\Psi}}{\partial \bar{I}_{1}^{2}}\left(\bar{I}_{1}, \bar{I}_{2}, c_{p}, c_{i}\right)>0 \quad(\alpha=1,2,3), \quad \forall \bar{I}_{1}, \bar{I}_{2} \geq 3,
\end{aligned}
$$

and hence that the effective stored-energy function (46) is strongly elliptic. For the case when $\Psi_{m}$ is merely strongly elliptic (i.e., it satisfies the weaker conditions (12)) but not convex in $I_{1}$, the effective stored-energy function (46) can still be shown to be strongly elliptic for small enough deformations, but it may lose strong ellipticity at sufficiently large values of deformation.

$i v$. In the limit of small deformations $\left(\bar{I}_{1}, \bar{I}_{2} \rightarrow 3\right), \mu_{0}=2 \Psi_{m}^{\prime}(3)=\mu_{m}$ to leading order in $\bar{I}_{1}$ and the stored-energy function (46) reduces asymptotically to

$$
\bar{\Psi}\left(\bar{I}_{1}, \bar{I}_{2}, c_{p}, c_{i}\right)=\bar{\mu} \operatorname{tr} \bar{\varepsilon}^{2}
$$

to leading order in the deformation measure $\bar{\varepsilon}=\left(\overline{\mathbf{F}}+\overline{\mathbf{F}}^{T}-2 \mathbf{I}\right) / 2$, where the effective shear modulus $\bar{\mu}=\bar{\mu}_{0}$ in (52) is defined implicitly by the remaining equation

$$
\mathcal{F}_{1}\left\{\bar{\mu}, \mu_{m}\right\}=0 .
$$

In general, equation (53) does not admit an explicit solution and thus $\bar{\mu}$ must be evaluated numerically. In this regard, it is useful to deduce that $\bar{\mu}$ is strictly positive, bounded from below by

$$
\bar{\mu} \geq\left\{\begin{array}{lll}
\mu_{m}+\frac{5\left[\left(3 c_{p}-2 c_{i}\right) \mu_{m}+2\left(c_{p}+c_{i}\right) \mu_{i}\right] \mu_{m}}{2\left[3\left(1-c_{p}\right)+2 c_{i}\right] \mu_{m}+4\left(1-c_{p}-c_{i}\right) \mu_{i}} & \text { if } \quad \mu_{i} \geq \mu_{m} \\
\mu_{m}+\frac{\left(2 \mu_{m}+3 \mu_{i}\right)\left[5 c_{p} \mu_{i}+2 c_{i}\left(\mu_{i}-\mu_{m}\right)\right]}{2\left[5\left(1-c_{p}\right)-2 c_{i}\right] \mu_{i}+4 c_{i} \mu_{m}} & \text { if } \quad \mu_{i}<\mu_{m}
\end{array},\right.
$$

and from above by

$$
\bar{\mu} \leq \frac{\mu_{m}}{\left(1-c_{p}-c_{i}\right)^{5 / 2}}
$$

for any choice of shear moduli $\mu_{m}, \mu_{i}>0$ and any choice of concentrations $c_{p}, c_{i} \geq 0$ with $c_{p}+c_{i} \leq 1$. Further, in the dilute limit as $c_{p}, c_{i} \rightarrow 0+$,

$$
\bar{\mu}=\bar{\mu}^{\text {dil }}=\mu_{m}+\frac{5 \mu_{m}}{2} c_{p}+\frac{5\left(\mu_{i}-\mu_{m}\right)\left(q_{1} \mu_{i}+q_{4} \mu_{m}\right) \mu_{m}}{2\left(q_{1} \mu_{i}^{2}+q_{2} \mu_{i} \mu_{m}+q_{3} \mu_{m}^{2}\right)} c_{i}
$$

to leading order in the concentration of particles $c_{p}$ and interphases $c_{i}$. 
The result (53) for $\bar{\mu}$ constitutes a generalization of the classical result of Brinkman-Roscoe (Roscoe, 1973) for the effective shear modulus of a suspension of polydisperse rigid spherical particles in rubber with perfect bonding (i.e., without interphases) between the particles and the rubber. Indeed, in the absence of interphases when $c_{i}=0$, equation (53) can be solved explicitly to render identically the well-known formula

$$
\bar{\mu}=\frac{\mu_{m}}{\left(1-c_{p}\right)^{5 / 2}} \text {. }
$$

$v$. The connection with the effective shear modulus $\bar{\mu}$ for isotropic distributions of polydisperse rigid spherical particles bonded through interphases as defined by equation (53) is not restricted to small deformations. Indeed, for the special case when the underlying matrix material is a Gaussian elastomer, $\Psi_{m}=\mu_{m} / 2\left[I_{1}-3\right], \mu_{0}=\mu_{m}$, and the effective stored-energy function (46) reduces to

$$
\bar{\Psi}\left(\bar{I}_{1}, \bar{I}_{2}, c_{p}, c_{i}\right)=\frac{\bar{\mu}}{2}\left[\bar{I}_{1}-3\right],
$$

which is seen to have the same functional form as the Gaussian matrix material, with the effective shear modulus $\bar{\mu}$ defined by (53). While exact and realizable in the limit of small deformations, for arbitrarily large deformations the effective stored-energy function (58) is not an exact realizable result for Gaussian elastomers filled with an isotropic distribution of rigid spherical particles of polydisperse sizes bonded through Gaussian interphases. Owing to its iterative construction process (see Section 4.1), however, it is expected to provide a very accurate approximation for this class of material systems. By the same token, the approximate effective stored-energy function (46) is also expected to describe very accurately the response of any such type of filled elastomer when the underlying matrix is a non-Gaussian elastomer, especially in the small and moderate deformation regimes. For large deformations, the result (46) is likely to be relatively less accurate for this class of material systems, as its variational construction process (see Section 4.2) entails that it corresponds to some sort of lower (upper) bound when the underlying matrix material has stronger (weaker) growth conditions than a Gaussian elastomer. These expectations are supported by comparisons with the FE simulations presented in Section 6.

vi. In the absence of interphases when $c_{i}=0$, equations (48) admit the explicit solution $\bar{\mu}_{0}=\mu_{0} /(1-$ $\left.c_{p}\right)^{5 / 2}, \mu_{0}=2 \Psi_{m}^{\prime}\left(\left[\bar{I}_{1}-3\right] /\left(1-c_{p}\right)^{7 / 2}+3\right)$, and the effective stored-energy function (46) reduces to the result of Lopez-Pamies et al. (2013b) for the effective stored-energy function of a suspension of polydisperse rigid spherical particles in rubber with perfect bonding between the particles and the rubber, namely,

$$
\bar{\Psi}\left(\bar{I}_{1}, \bar{I}_{2}, c_{p}, 0\right)=\left(1-c_{p}\right) \Psi_{m}\left(\frac{\bar{I}_{1}-3}{\left(1-c_{p}\right)^{7 / 2}}+3\right) .
$$

vii. In the limit of rigid interphases when $\mu_{i}=+\infty$, equations (48) similarly admit the explicit solution $\bar{\mu}_{0}=\mu_{0} /\left(1-c_{p}-c_{i}\right)^{5 / 2}, \mu_{0}=2 \Psi_{m}^{\prime}\left(\left[\bar{I}_{1}-3\right] /\left(1-c_{p}-c_{i}\right)^{7 / 2}+3\right)$, and the effective stored-energy function (46) reduces to

$$
\bar{\Psi}\left(\bar{I}_{1}, \bar{I}_{2}, c_{p}, c_{i}\right)=\left(1-c_{p}-c_{i}\right) \Psi_{m}\left(\frac{\bar{I}_{1}-3}{\left(1-c_{p}-c_{i}\right)^{7 / 2}}+3\right) .
$$

This result also agrees with the effective stored-energy function of Lopez-Pamies et al. (2013b) for a suspension of polydisperse rigid spherical particles in rubber with perfect bonding between the particles and the rubber, since in this limiting case there are actually no interphases but instead a distribution of rigid particles with total concentration $c_{p}+c_{i}$.

\section{FE simulations of filled elastomers undergoing large deformations}

With the aim of gaining further insight, in Section 6 we confront the above-developed theoretical results to full 3D FE simulations of the large-deformation response of Gaussian and non-Gaussian elastomers filled 
by random isotropic distributions of rigid spherical particles that are bonded through constant-thickness interphases. In particular, following common practice (see, e.g., Gusev, 1997; Michel et al., 1999), we consider infinite periodic media made up of the repetition of unit cells that contain a random distribution of a large but finite number of particles, as dictated by a sequential adsorption algorithm. In order to probe the effect that particle polydispersity plays on the overall response of filled elastomers with interphases ${ }^{6}$, we consider distributions with both, particles of the same (monodisperse) size and particles of different (polydisperse) sizes. The details of the simulations are as follows.

\subsection{Monodisperse microstructures}
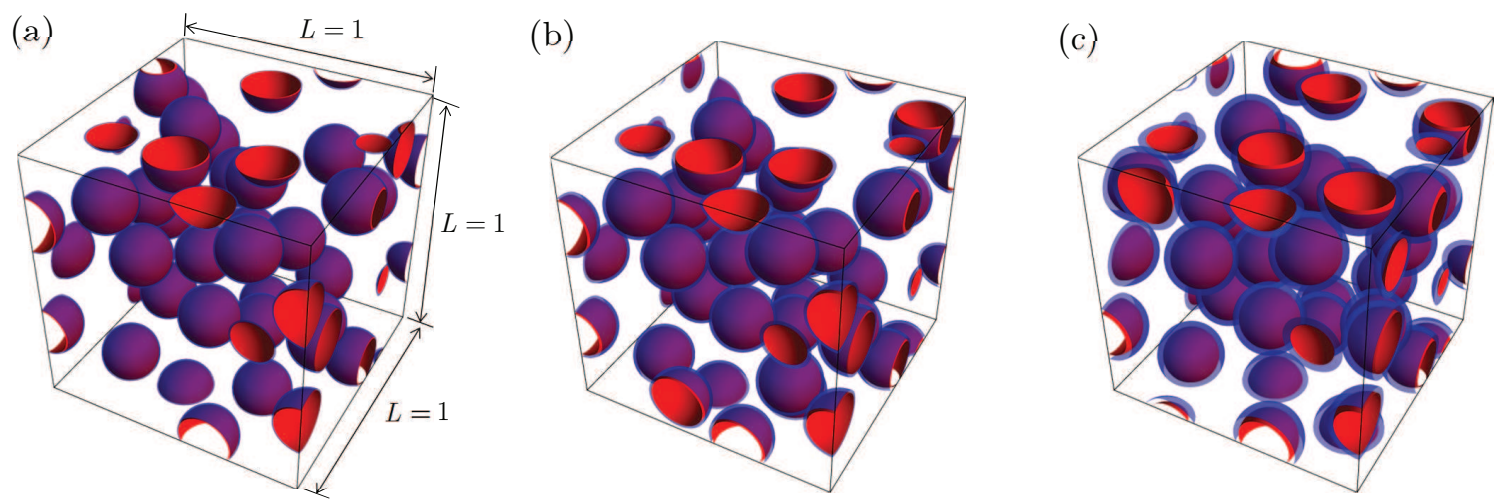

Figure 11: Representative unit cells containing a random distribution of $N=30$ monodisperse spherical particles of radius $r$ at concentration $c_{p}=0.15$ that are surrounded by interphases of three different constant thicknesses $t$ : (a) $t=0.05 r$, (b) $t=0.1 r$, and (c) $t=0.2 r$.

The monodisperse microstructures are constructed using a random sequential adsorption algorithm in which the sequential addition of spherical particles, of the same radius $r$ with surrounding interphases of the same constant thickness $t$, is constrained so that the distance between a given interphase with other interphases and with the boundaries of the unit cell - chosen here to be a cube of unit side $L=1$ - take a minimum value that allows for an adequate spatial discretization (see, e.g., Segurado and Llorca, 2002; Lopez-Pamies et al., 2013b), namely:

- The center-to-center distance between a new particle $i$ and any previously accepted one $j=1,2, \ldots, i-1$ has to exceed the minimum value $s_{1}=2(r+t)\left(1+d_{1}\right)$, where the offset distance $d_{1}$ is fixed here at $d_{1} \geq 0.03$. The condition to be checked at each step of the algorithm takes then the form

$$
\left\|\mathbf{X}^{i}-\mathbf{X}^{j}-\mathbf{h}\right\| \geq s_{1}
$$

where $\mathbf{X}^{i}\left(\mathbf{X}^{j}\right)$ denotes the location of the center of particle $i(j)$ and $\mathbf{h}$ is a vector with entries $0, L$, or $-L$ for each of its three Cartesian components with respect to the principal axes of the cubic unit cell.

- The outermost surface of any interphase should be sufficiently distant from the boundaries of the unit cell as enforced by the inequalities

$$
\left|X_{\alpha}^{i}-r-t\right| \geq s_{2} \quad \text { and } \quad\left|X_{\alpha}^{i}+r+t-L\right| \geq s_{2} \quad(\alpha=1,2,3),
$$

where $s_{2}=d_{2}(r+t)$ with $d_{2}$ being fixed here at 0.05 .

\footnotetext{
${ }^{6}$ In the absence of interphases, Lopez-Pamies et al. (2013b) have shown that the effect of polydispersity, rather remarkably, is negligible up to relative large concentrations of particles in the order of $c_{p}=0.3$.
} 
For this class of monodisperse microstructures, we note that the radius $r$ of the particles and the thickness $t$ of the surrounding interphases are related to the total number of particles $N$, particle concentration $c_{p}$, and interphase concentration $c_{i}$ via

$$
r=L\left(\frac{3 c_{p}}{4 \pi N}\right)^{1 / 3} \quad \text { and } \quad \frac{t}{r}=\left(1+\frac{c_{i}}{c_{p}}\right)^{1 / 3}-1 .
$$

Previous results based on this approach - for linear (Segurado and Llorca, 2002) as well as for nonlinear (Lopez-Pamies et al., 2013b) problems - have indicated that $N=30$ particles is, in general, sufficient to approximate isotropic symmetry. Figure 11 depicts representative examples of such unit cells with $N=30$ particles at concentration $c_{p}=0.15$ for three different interphase thicknesses $t$ : (a) $t=0.05 r$, (b) $t=0.1 r$, and (c) $t=0.2 r$.

\subsection{Polydisperse microstructures}
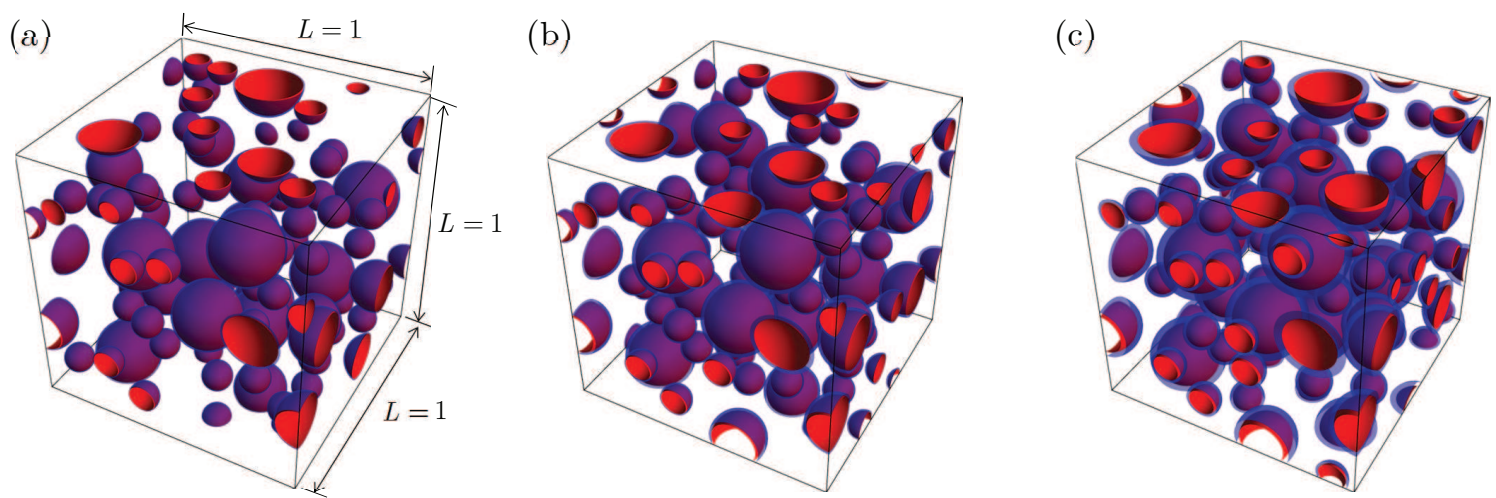

Figure 12: Representative unit cells containing a random distribution of $N=80$ spherical particles of three different radii at concentration $c_{p}=0.15$ that are surrounded by interphases with three different constant thickness-to-particleradius ratios: (a) $t / r=0.05$, (b) $t / r=0.1$, and (c) $t / r=0.2$.

The polydisperse microstructures are constructed by means of a similar constrained adsorption algorithm. The focus is on polydisperse microstructures with three different families of particle sizes such that - consistent with the assumptions made in the derivation of the theoretical results of Section 4 - the interphase-thickness-to-particle-radius ratio is the same for all particles. While there is no distinct rule for the creation of such microstructures and the possibilities are many, we consider for definiteness the following procedure:

- Three different families of spherical particles with radii $r^{(I)}$ and respective concentrations $c_{p}^{(I)}(I=$ $1,2,3)$, surrounded by interphases with thicknesses $t^{(I)}$ and respective concentrations $c_{i}^{(I)}(I=1,2,3)$, are utilized such that

$$
\begin{aligned}
& \left\{r^{(1)}, r^{(2)}, r^{(3)}\right\}=\left\{r, \frac{7}{9} r, \frac{4}{9} r\right\} \quad \text { with } \quad r=L\left(\frac{3 c_{p}^{(1)}}{4 \pi N^{(1)}}\right)^{1 / 3}, \\
& \left\{c_{p}^{(1)}, c_{p}^{(2)}, c_{p}^{(3)}\right\}=\left\{0.5 c_{p}, 0.25 c_{p}, 0.25 c_{p}\right\} \quad \text { with } \quad c_{p}^{(1)}+c_{p}^{(2)}+c_{p}^{(3)}=c_{p}, \\
& \left\{\frac{t^{(1)}}{r^{(1)}}, \frac{t^{(2)}}{r^{(2)}}, \frac{t^{(3)}}{r^{(3)}}\right\}=\left\{\frac{t}{r}, \frac{t}{r}, \frac{t}{r}\right\} \quad \text { with } \quad \frac{t}{r}=\left(1+\frac{c_{i}^{(1)}}{c_{p}^{(1)}}\right)^{1 / 3}-1,
\end{aligned}
$$

where $N^{(1)}$ is the number of particles with the largest radius and thickest surrounding interphase, $r^{(1)}=r$ and $t^{(1)}=t$, in the unit cell. 
- The microstructures are generated sequentially by first adding the particles with the largest radius $r^{(1)}=r$ and desired thickness $t^{(1)}=t$, until the particle concentration reaches the value $c_{p}^{(1)}=0.5 c_{p}$, subsequently adding particles with radius $r^{(2)}$ and surrounding interphases of thickness $t^{(2)}=r^{(2)} t / r$ until $c_{p}^{(1)}+c_{p}^{(2)} \approx 0.75 c_{p}$, and finally adding particles with the smallest radius $r^{(3)}$ and smallest interphase thickness $t^{(3)}=r^{(3)} t / r$ until $c_{p}^{(1)}+c_{p}^{(2)}+c_{p}^{(3)} \approx c_{p}$. In following this construction process, we note that a target concentration $c_{p}$ (similarly for a target concentration $c_{i}$ if preferred over a target ratio $t / r$ ) can only be achieved approximately up to a small error that depends on the various choices of the parameters. To guarantee adequate spatial discretization, the randomly generated placements of the centers of the particles are enforced to satisfy constraints analogous to those enforced for the case of monodisperse microstructures, cf. inequalities (61) and (62).

In this work we utilize $N^{(1)}=10$ which results in unit cells containing a total of $N=80$ particles that prove to be sufficiently isotropic for our purposes. Figure 12 depicts representative examples of such unit cells with $N=80$ particles of three different radii at concentration $c_{p}=0.15$ for three different interphasethickness-to-particle-radius ratios: (a) $t / r=0.05$, (b) $t / r=0.1$, and (c) $t / r=0.2$.

\subsection{Spatial discretization, particle material behavior, and computation of the overall response}

The discretizations of the microstructures are carried out by means of the mesh generator code Netgen (Schöberl, 1997). Hybrid isoparametric 10-node quadratic tetrahedral elements with constant pressure proved to deliver accurate results, and thus were selected to carry out the calculations. Figure 13 displays three representative meshes of increasing refinement for a monodisperse microstructure with concentrations of particles $c_{p}=0.15$ and interphases $c_{i}=0.1$. Mesh sensitivity analyses revealed that meshes containing about 200,000 elements, such as the one shown in Fig. 13(c), are refined enough to deliver accurate results for all the cases considered here.
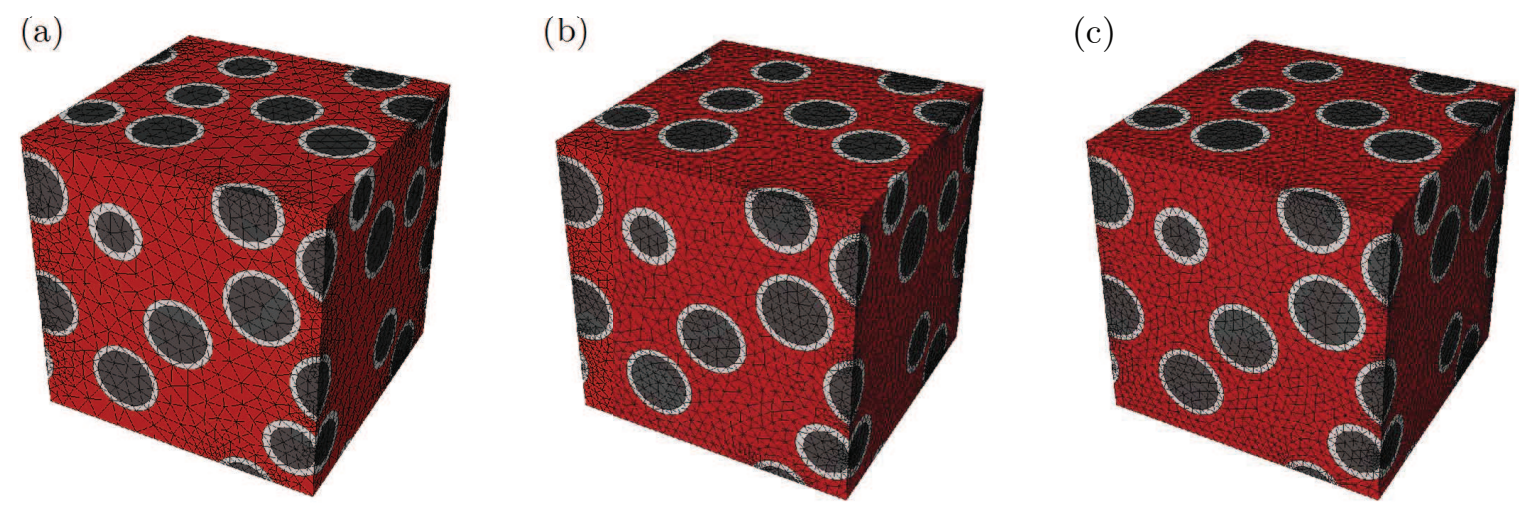

Figure 13: Three progressively refined meshes, with about (a) 35,000, (b) 90,000, and (c) 180,000 elements, for a distribution of monodisperse particles with concentration $c_{p}=0.15$ and interphase concentration $c_{i}=0.1$.

Within the utilized formulation, the perfectly rigid behavior (4) of the particles is modeled approximately by means of a very (but not infinitely) stiff material. Here, for definiteness, we model the particles as a Gaussian elastomer with stored-energy function

$$
W_{p}^{F E}(\mathbf{F})=\left\{\begin{array}{ll}
\frac{\mu_{p}^{F E}}{2}\left[I_{1}-3\right] & \text { if } J=1 \\
+\infty & \text { otherwise }
\end{array},\right.
$$

where the parameter $\mu_{p}^{F E}$ is set to be four orders of magnitude larger than the shear modulus of the underlying matrix material, i.e., $\mu_{p}^{F E}=10^{4} \times \mu_{m}$. 
By virtue of their periodicity, the computation of the effective stored-energy function (9) for any of the above-defined classes of filled elastomers amounts to subjecting their defining cubic unit cells to the periodic boundary conditions

$$
\begin{aligned}
& u_{\alpha}\left(0, X_{2}, X_{3}\right)-u_{\alpha}\left(L, X_{2}, X_{3}\right)=\left(\bar{F}_{\alpha 1}-\delta_{\alpha 1}\right) L, \\
& u_{\alpha}\left(X_{1}, 0, X_{3}\right)-u_{\alpha}\left(X_{1}, L, X_{3}\right)=\left(\bar{F}_{\alpha 2}-\delta_{\alpha 2}\right) L, \\
& u_{\alpha}\left(X_{1}, X_{2}, 0\right)-u_{\alpha}\left(X_{1}, X_{2}, L\right)=\left(\bar{F}_{\alpha 3}-\delta_{\alpha 3}\right) L
\end{aligned}
$$

$(\alpha=1,2,3)$ in terms of the displacement field $\mathbf{u}=\mathbf{x}-\mathbf{X}$, and computing the resulting total elastic energy per unit undeformed volume of the unit cell. In expression (68), the components $u_{\alpha}$ and $X_{\alpha}(\alpha=1,2,3)$ refer to a Cartesian frame of reference with origin placed at a corner of the cubic unit cell whose axes $\left\{\mathbf{e}_{\alpha}\right\}$ are aligned with the principal axes of the cubic unit cell (see Fig. 13), and $\delta_{\alpha \beta}$ denotes the Kronecker delta.

\subsection{Assessment of the simulations}

Because of the finite number of particles $-N=30$ for the monodisperse and $N=80$ for the polydisperse microstructures - included per unit cell, the microstructures simulated here are (not exactly but) only approximately isotropic. In order to assess the isotropy of each realization that is constructed, we examine the co-axiallity between the average Cauchy stress tensor $\overline{\mathbf{T}} \doteq \overline{\mathbf{S}} \overline{\mathbf{F}}^{T}$ and the average left Green-Cauchy strain tensor $\overline{\mathbf{B}} \doteq \overline{\mathbf{F}} \overline{\mathbf{F}}^{T}$ under three types of loading conditions: $(i)$ axisymmetric tension where $\overline{\mathbf{F}}=$ $\bar{\lambda} \mathbf{e}_{1} \otimes \mathbf{e}_{1}+\bar{\lambda}^{-1 / 2}\left(\mathbf{e}_{2} \otimes \mathbf{e}_{2}+\mathbf{e}_{3} \otimes \mathbf{e}_{3}\right)$ with $\bar{\lambda} \geq 1,($ ii $)$ axisymmetric compression where $\overline{\mathbf{F}}=\bar{\lambda} \mathbf{e}_{1} \otimes \mathbf{e}_{1}+$ $\bar{\lambda}^{-1 / 2}\left(\mathbf{e}_{2} \otimes \mathbf{e}_{2}+\mathbf{e}_{3} \otimes \mathbf{e}_{3}\right)$ with $\bar{\lambda} \leq 1$, and (iii) simple shear where $\overline{\mathbf{F}}=\mathbf{I}+\bar{\gamma} \mathbf{e}_{1} \otimes \mathbf{e}_{2}$ with $\bar{\gamma} \geq 0$. Only microstructures for which the maximum difference between any two corresponding principal axes of $\overline{\mathbf{T}}$ and $\overline{\mathbf{B}}$ is less than 0.05 radians for all three loadings are admitted as approximately isotropic.

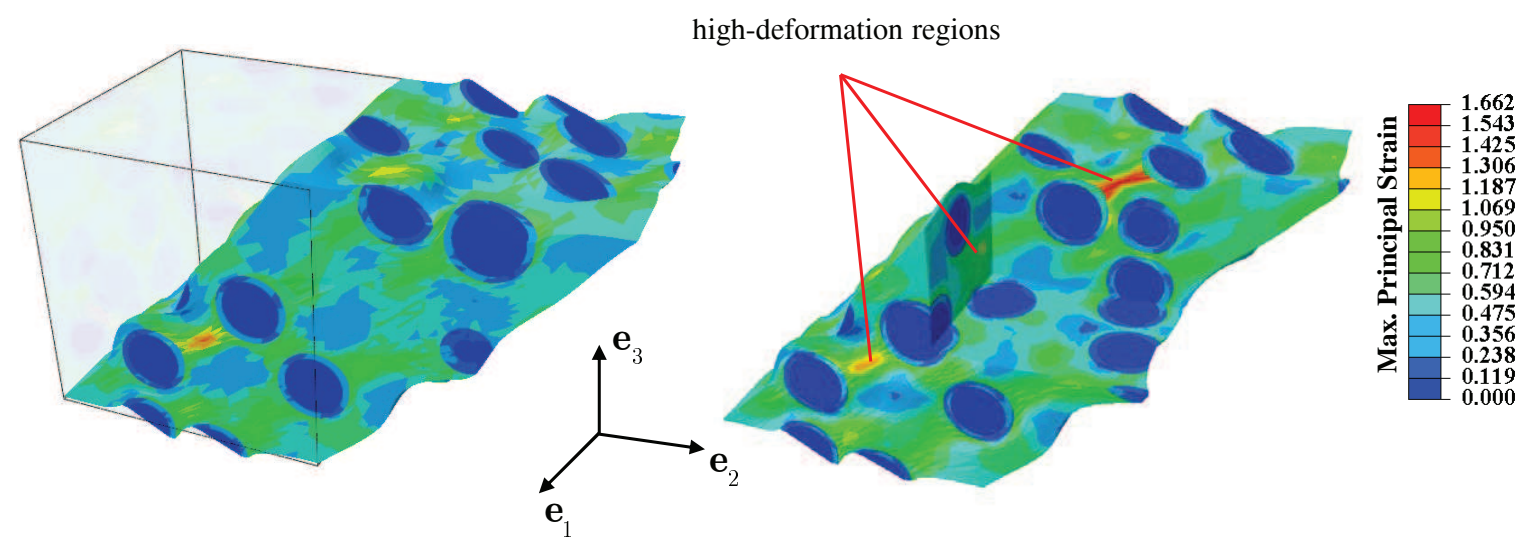

Figure 14: Contour plots of the maximum principal logarithmic strain for a monodisperse realization with $c_{p}=0.15$, $c_{i}=0.05$, Gaussian elastomeric matrix with shear modulus $\mu_{m}=1 \mathrm{MPa}$, and $\mu_{i}=5 \mathrm{MPa}$ subjected to a macroscopic simple shear strain of $\bar{\gamma}=0.91$; the undeformed configuration is also depicted for comparison purposes. Part (b) shows an inside view of three pairs of particles/interphases in between which the matrix material is highly deformed.

All FE results to be presented in the next section correspond to the average of three different realizations, all of which are approximately isotropic in the sense described in the preceding paragraph. The computations are carried out in the commercial code ABAQUS by following an incremental loading path. We utilize the default dual convergence criterion in this code (see ABAQUS Version 6.11 Documentation), namely, the permissible ratio of the largest solution correction to the largest corresponding incremental solution is set at $|\Delta \mathbf{u}| /\left|\mathbf{u}_{\max }\right|=10^{-2}$, while the permissible ratio of the largest residual to the corresponding average force norm is set at $R_{t o l}=5 \times 10^{-3}$. Whenever one of these criteria is not satisfied the computations are stopped. This typically happens whenever the elements in between two adjacent interphases become exceedingly distorted because of the locally large deformations involved. 
Figure 14 presents an example of large local deformations in between interphases for the case of a monodisperse realization with $c_{p}=0.15, c_{i}=0.05$, Gaussian elastomeric matrix with shear modulus $\mu_{m}=$ $1 \mathrm{MPa}$, and $\mu_{i}=5 \mathrm{MPa}$ under simple shear. Part (a) shows contour plots of the maximum principal logarithmic strain at an overall shear strain level of $\bar{\gamma}=0.91$; the initial undeformed geometry is also depicted for comparison purposes. The deformation contours are seen to be highly heterogeneous with principal logarithmic strains as large as 1.66 within regions between interphases. In part (b), an inside view is shown of three regions of strong particle/interphase interaction and high local strains that lead to significant mesh distortion and therefore problems with the numerical convergence of the FE calculations. In principle, re-meshing of these regions should allow to reach further overall deformations, but this is not pursued here.

\section{Sample results and comparisons with FE simulations}

Sample results are now presented that provide quantitative insight into the proposed theoretical result (46) and that, at the same time, serve to reveal the importance of the various microscopic quantities (the nonlinear elastic behavior of the matrix $\Psi_{m}$, the concentration of the particles $c_{p}$, the concentration of the interphases $c_{i}$, and the stiffness of the interphases $\mu_{i}$ ) on the macroscopic response of filled elastomers. We begin in Section 6.1 by presenting results for the linear elastic response of filled elastomers in the smalldeformation regime. These are followed in Section 6.2 by results for the large-deformation response of filled Gaussian elastomers. Finally, in Section 6.3 we present results for a filled elastomer wherein the matrix is characterized by the non-Gaussian stored-energy function

$$
\Psi_{m}\left(I_{1}\right)=\frac{3^{1-\alpha_{1}}}{2 \alpha_{1}} \mu_{1}\left[I_{1}^{\alpha_{1}}-3^{\alpha_{1}}\right]+\frac{3^{1-\alpha_{2}}}{2 \alpha_{2}} \mu_{2}\left[I_{1}^{\alpha_{2}}-3^{\alpha_{2}}\right]
$$

with $\mu_{1}=0.032 \mathrm{MPa}, \mu_{2}=0.3 \mathrm{MPa}, \alpha_{1}=3.837, \alpha_{2}=0.559$, corresponding to a model that describes accurately the nonlinear elastic response of a standard silicone rubber over large ranges of deformations (Lopez-Pamies, 2010a).

\subsection{Linear elastic results}

In the limit of small deformations (see remark $(i v)$ in Section 4.2), the effective stored-energy function (46) reduces to (52) and hence is completely characterized by the effective shear modulus $\bar{\mu}$ defined by equation (53). Figure 15 shows results for the normalized effective shear modulus $\bar{\mu} / \mu_{m}$ for various values of the interphase-thickness-to-particle-radius ratio ${ }^{7} t / r$ and interphase stiffness $\mu_{i} / \mu_{m}$, all as functions of the concentration of particles $c_{p}$. Results are also presented for the FE simulations described in Section 5 for monodisperse (Figs. 15(a) and (c)) and polydisperse (Figs. 15(b) and (d)) microstructures.

As expected, an immediate observation from Fig. 15 is that the addition of rigid particles increases significantly the overall stiffness of elastomers. Remarkably, the presence of interphases $\left(\right.$ with $\left.\mu_{i}>\mu_{m}\right)$ is seen to also have a comparable stiffening effect. We note that the stiffening granted by the interphases is highly more sensitive to the value of their thickness $t / r$ than to the value of their stiffness $\mu_{i} / \mu_{m}$. Indeed, Figs. $15(\mathrm{c})$ and $(\mathrm{d})$ show that interphases that are just 5 times stiffer than the matrix $\left(\mu_{i} / \mu_{m}=5\right)$ already grant an increase in overall stiffness that is similar to that granted by rigid interphases $\left(\mu_{i}=+\infty\right)$. On the other hand, Figs. 15(a) and (b) show that moderate increases in the thickness of the interphases from $t / r=0$, to $t / r=0.05$, to $t / r=0.1$, to $t / r=0.2$, consistently lead to larger enhancements of the overall stiffness. The practical implications of these results are far reaching as they suggest that when dealing with conventional filled elastomers - where interphases are typically in the order of 10 times stiffer than the matrix material (see, e.g., Qu et al., 2011) — their macroscopic response is by and large unaffected by the constitutive complexity of the underlying interphases (in terms of heterogeneity, anisotropy, and non-linearity) and de facto simply dependent on their average stiffness and size.

\footnotetext{
${ }^{7}$ Throughout this section, we shall favor writing the content of interphases in terms of the ratio $t / r=\left(1+c_{i} / c_{p}\right)^{1 / 3}-1$, instead of directly in terms of their concentration $c_{i}$.
} 


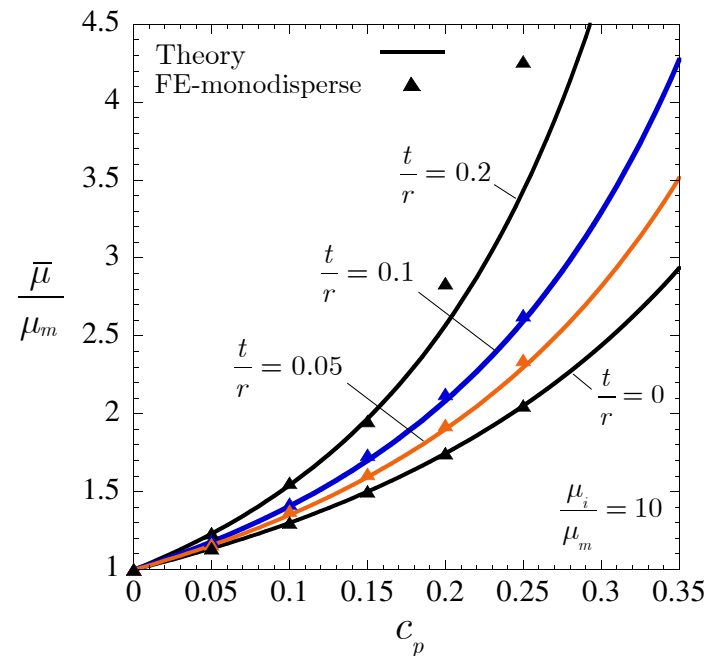

(a)

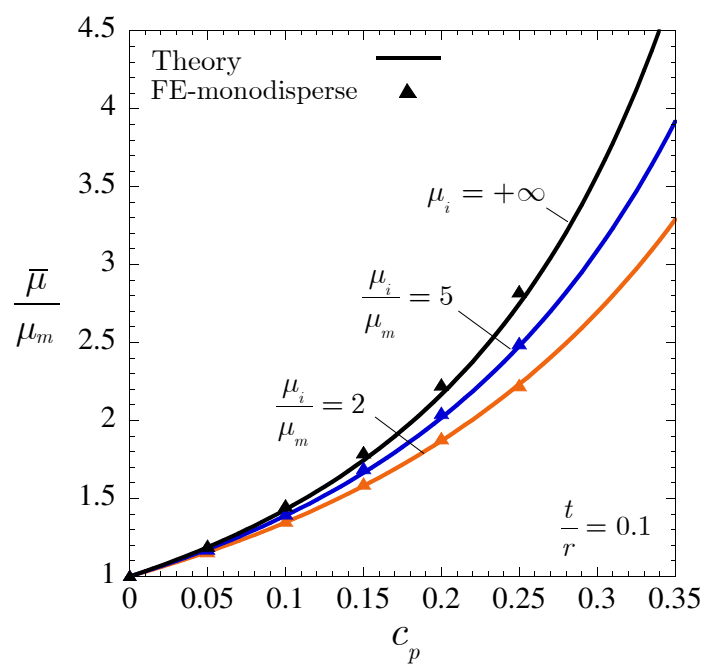

(c)

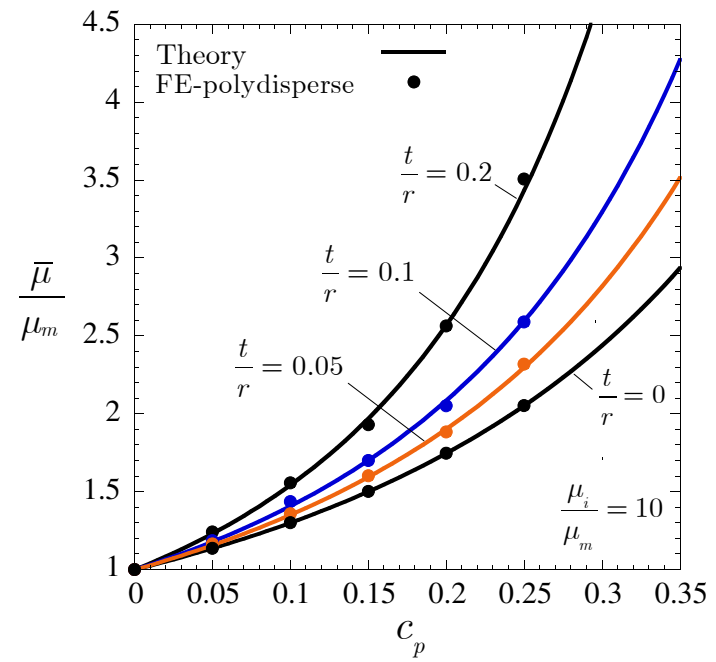

(b)

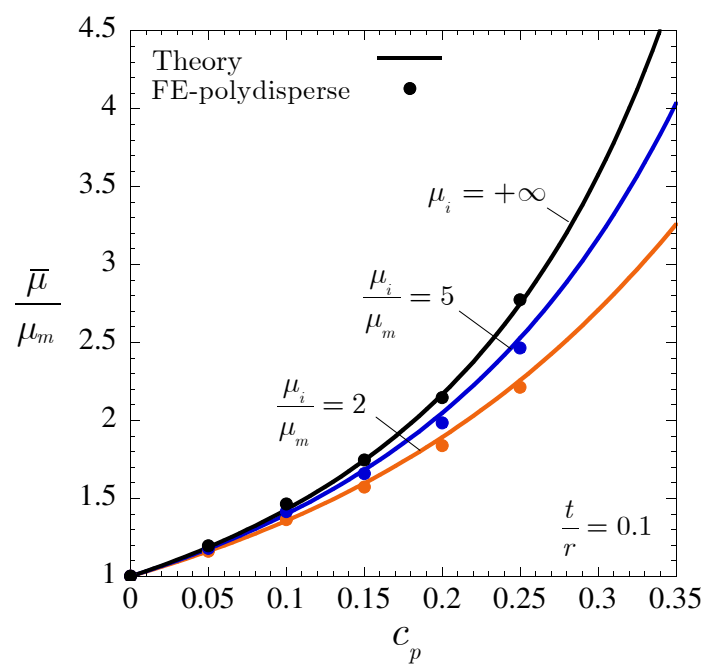

(d)

Figure 15: The normalized effective shear modulus $\bar{\mu} / \mu_{m}$ of an isotropic incompressible elastomer, with shear modulus $\mu_{m}$, filled with an isotropic distribution of rigid spherical particles bonded through isotropic incompressible interphases with various shear moduli $\mu_{i} / \mu_{m}$ and constant thickness-to-particle-radius ratios $t / r$. Results are shown for the theoretical result (53) compared to FE simulations for distributions of (a, c) monodisperse and (b, d) polydisperse particles, all as functions of the concentration of particles $c_{p}$.

While the effective shear modulus $\bar{\mu}$ defined by equation (53) is exact for infinitely polydisperse particles, Figs. 15(b) and (d) show it to be in good agreement with the FE results for microstructures with only three families of particle sizes for the entire range of particle concentrations and interphase thicknesses considered, $c_{p} \in[0,0.25]$ and $t / r \in[0,0.2]$. More remarkably, Figs. 15(a) and (c) show the theoretical effective shear modulus to also be in good agreement with the FE results for monodisperse particles up to the relatively high particle concentration $c_{p}=0.2$ with relatively large interphase thickness $t / r=0.2$. Consistent with earlier results for suspensions of particles in rubber without interphases (Lopez-Pamies, 2013b), these favorable comparisons suggest that polydispersity does not play a role in the response of filled elastomers for particle concentrations and interphase thicknesses sufficiently below the percolation limit. 


\subsection{Results for filled Gaussian elastomers}

Figure 16 presents results for the large-deformation response of a filled Gaussian elastomer with particle concentration $c_{p}=0.15$ under: (a) uniaxial compression, (b) uniaxial tension, (c) pure shear, and (d) simple shear. The constitutive stress-deformation relations for these loading conditions read as (see remark $(v)$ in Section 4.2)

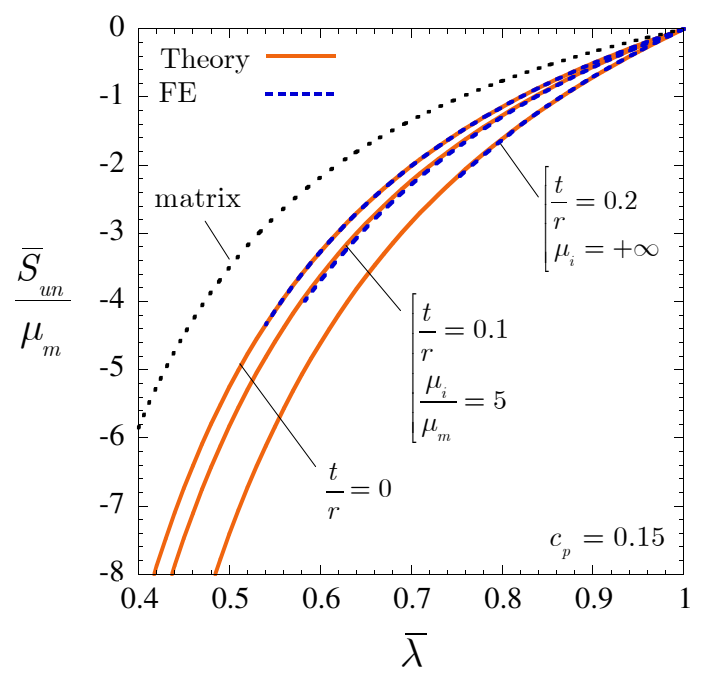

(a)

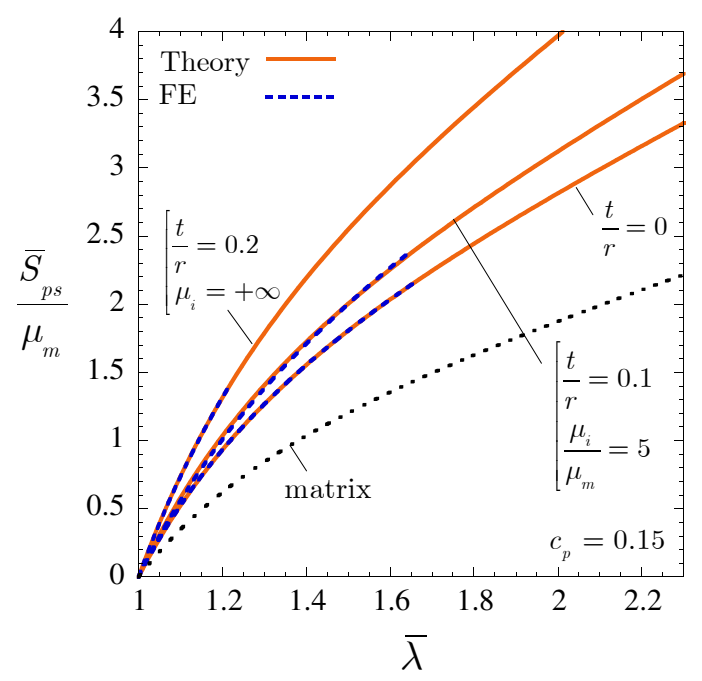

(c)

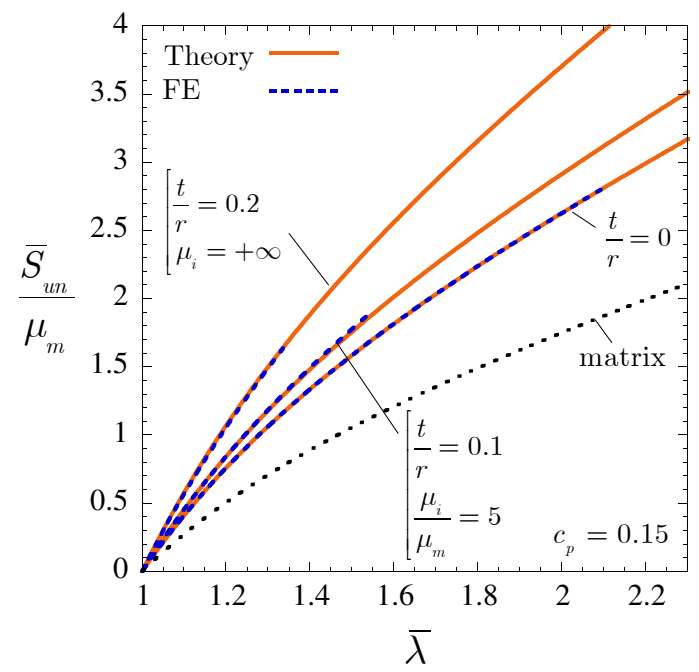

(b)

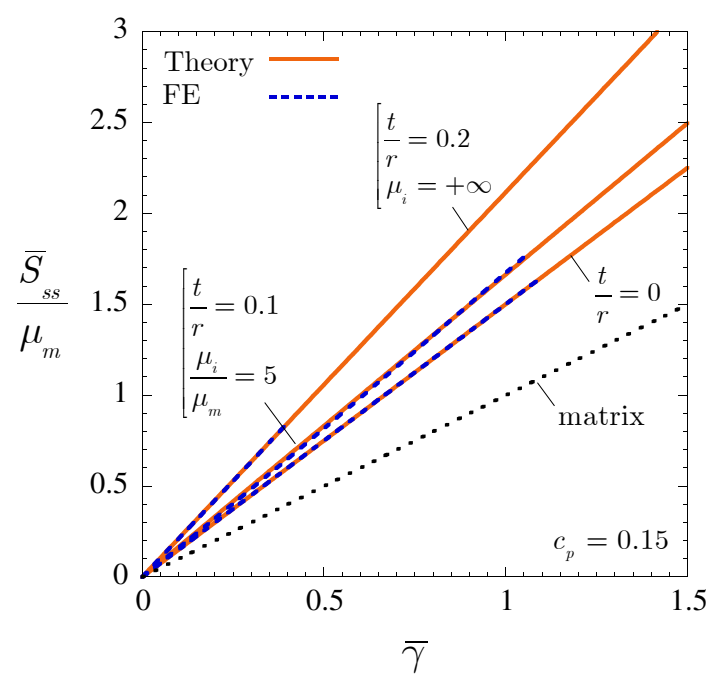

(d)

Figure 16: Macroscopic response of a filled Gaussian elastomer with particle concentration $c_{p}=0.15$ under: (a) uniaxial compressive, (b) uniaxial tensile, (c) pure shear, and (d) simple shear loading conditions, as characterized by the proposed theory, $c f$. expressions (70), (71), (72). Results are shown for three different types of interphases: (i) no interphases $t / r=0,($ ii $)$ interphases with moderate shear modulus $\mu_{i} / \mu_{m}=5$ and moderate thickness $t / r=0.1$, and $($ iii $)$ rigid interphases $\mu_{i}=+\infty$ with relatively large thickness $t / r=0.2$. The dashed lines in the plots correspond to results from the FE simulations, while the dotted lines correspond to the response of the unfilled Gaussian elastomer. 
- Uniaxial loading $\left(\bar{\lambda}_{1}=\bar{\lambda}, \bar{\lambda}_{2}=\bar{\lambda}_{3}=\bar{\lambda}^{-1 / 2}\right.$ with $\left.\bar{\tau}_{2}=\bar{\tau}_{3}=0\right)$ :

$$
\bar{S}_{u n}=\bar{\lambda}^{-1} \bar{\tau}_{1}=\frac{\mathrm{d} \bar{\Psi}}{\mathrm{d} \bar{\lambda}}=\bar{\mu}\left[\bar{\lambda}-\bar{\lambda}^{-2}\right]
$$

- Pure shear $\left(\bar{\lambda}_{1}=\bar{\lambda}, \bar{\lambda}_{2}=\bar{\lambda}^{-1}, \bar{\lambda}_{3}=1\right.$ with $\left.\bar{\tau}_{2}=0\right)$ :

$$
\bar{S}_{p s}=\bar{\lambda}^{-1} \bar{\tau}_{1}=\frac{\mathrm{d} \bar{\Psi}}{\mathrm{d} \bar{\lambda}}=\bar{\mu}\left[\bar{\lambda}-\bar{\lambda}^{-3}\right]
$$

- Simple shear $\left(\bar{\lambda}_{1}=\left(\bar{\gamma}+\sqrt{\bar{\gamma}^{2}+4}\right) / 2, \bar{\lambda}_{2}=\bar{\lambda}_{1}^{-1}, \bar{\lambda}_{3}=1\right)$ :

$$
\bar{S}_{s s}=\frac{\mathrm{d} \bar{\Psi}}{\mathrm{d} \bar{\gamma}}=\bar{\mu} \bar{\gamma}
$$

where $\bar{S}_{u n}, \bar{S}_{p s}, \bar{S}_{s s}$ denote first Piola-Kirchhoff stress measures, $\bar{\tau}_{1}, \bar{\tau}_{2}, \bar{\tau}_{3}$ have been introduced to denote the macroscopic principal Cauchy stresses, and, again, the effective shear modulus $\bar{\mu}$ is implicitly defined by equation (53). Three different types of interphases are considered: (i) no interphases $t / r=0,(i i)$ interphases with moderate shear modulus $\mu_{i} / \mu_{m}=5$ and moderate thickness $t / r=0.1$, and (iii) rigid interphases $\mu_{i}=+\infty$ with relatively large thickness $t / r=0.2$. Corresponding FE results are included in the figure for comparison purposes. No distinction is made of whether the particles are monodisperse or polydisperse since, somewhat remarkably, both classes of simulated microstructures exhibit essentially the same large-deformation response. This is consistent with the linear elastic results of Fig. 15, where the monodisperse and polydisperse FE simulations render practically identical effective shear moduli for particle concentrations below $c_{p}=0.2$ with interphase-thickness-to-particle-radius ratios below $t / r=0.2$.

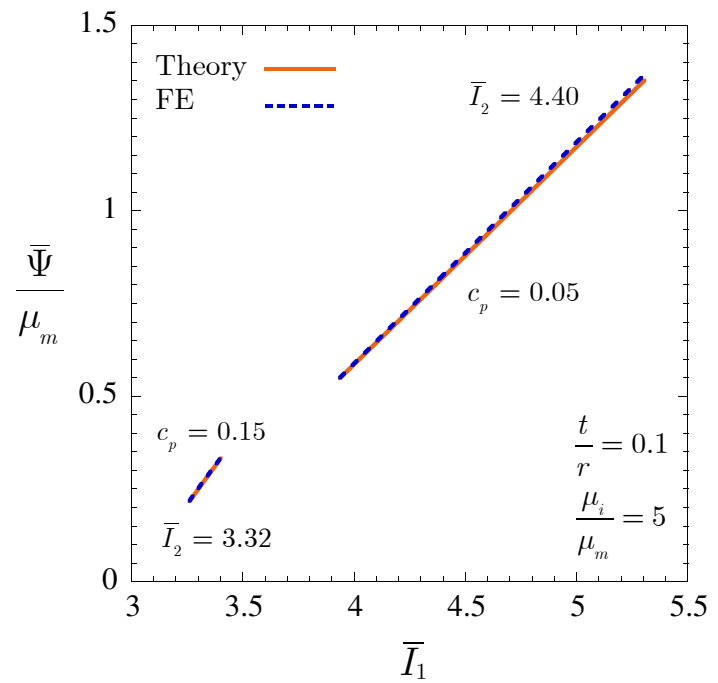

(a)

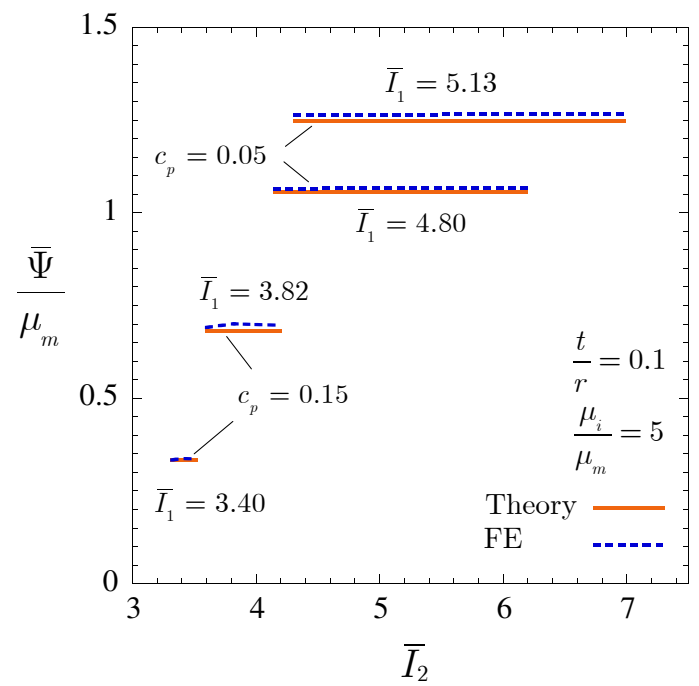

(b)

Figure 17: Comparison of the effective stored-energy function (58) for a filled Gaussian elastomer with corresponding FE simulations. The results are shown in terms of the principal invariants $\bar{I}_{1}$ and $\bar{I}_{2}$ for interphase-thickness-toparticle-radius ratio $t / r=0.1$, interphase shear modulus $\mu_{i} / \mu_{m}=5$, and two values of concentration of particles, $c_{p}=0.05$ and 0.15 . Part (a) shows results for fixed values of $\bar{I}_{2}$ as functions of $\bar{I}_{1}$, while part (b) shows results for fixed values of $\bar{I}_{1}$ as functions of $\bar{I}_{2}$.

It is plain from Fig. 16 that the overall large-deformation response of the Gaussian elastomer is stiffened significantly by the addition of rigid particles for all loading conditions. The figure also makes it plain that 
the presence of interphases (with $\mu_{i}>\mu_{m}$ ) produces levels of stiffness enhancement that are comparable to those produced by the particles themselves. As it was the case for small deformations, the increase in stiffness generated by the interphases is more dependent on their thickness $t / r$ than on their stiffness $\mu_{i} / \mu_{m}$. Another important observation from Fig. 16 is that the theoretical predictions and FE results are in good qualitative and quantitative agreement for all loading conditions.

To further probe the connections between the proposed theory and the FE simulations, Fig. 17 compares their elastic energies $\bar{\Psi} / \mu_{m}$, normalized by the initial shear modulus $\mu_{m}$ of the underlying Gaussian matrix, as functions of the principal invariants $\bar{I}_{1}$ and $\bar{I}_{2}$. Part (a) of the figure shows $\bar{\Psi} / \mu_{m}$ for fixed values of the second invariant $\bar{I}_{2}=3.32$ for $c_{p}=0.15$ and $\bar{I}_{2}=4.40$ for $c_{p}=0.05$ as functions of $\bar{I}_{1}$, while part (b) shows results for fixed values of the first invariant $\bar{I}_{1}=3.40,3.82$ for $c_{p}=0.15$ and $\bar{I}_{1}=4.80,5.13$ for $c_{p}=0.05$ as functions of $\bar{I}_{2}$.

The main observation from Fig. 17 is that the FE results are approximately linear in the first invariant $\bar{I}_{1}$ and independent of the second invariant $\bar{I}_{2}$. This behavior is in accordance with that of the theory, corroborating that both results are very much identical in their functional form. The fact that the macroscopic behavior of filled Gaussian elastomers is functionally the same - i.e., linear in $\bar{I}_{1}$ and independent of $\bar{I}_{2}$ - as that of its underlying Gaussian matrix and interphases is of note. Indeed, as already mentioned in the discussion of Fig. 7, the functional character of the average behavior of nonlinear material systems is in general substantially different from that of its constituents, but that is not the case here.

\subsection{Results for a filled silicone elastomer}

Finally, Fig. 18 shows results for the large-deformation response of a filled non-Gaussian elastomer wherein the underlying matrix material is a typical silicone rubber characterized here by the stored-energy function (69) with $\mu_{1}=0.032 \mathrm{MPa}, \mu_{2}=0.3 \mathrm{MPa}, \alpha_{1}=3.837, \alpha_{2}=0.559$, and thus initial shear modulus $\mu_{m}=\mu_{1}+\mu_{2}=0.332 \mathrm{MPa}$. In this case, the proposed theoretical effective stored-energy function (46) specializes to

$\bar{\Psi}\left(\bar{I}_{1}, \bar{I}_{2}, c_{p}, c_{i}\right)=\left(1-c_{p}-c_{i}\right)\left[\frac{3^{1-\alpha_{1}}}{2 \alpha_{1}} \mu_{1}\left[\mathcal{I}_{1}^{\alpha_{1}}-3^{\alpha_{1}}\right]+\frac{3^{1-\alpha_{2}}}{2 \alpha_{2}} \mu_{2}\left[\mathcal{I}_{1}^{\alpha_{2}}-3^{\alpha_{2}}\right]\right]+\frac{\bar{\mu}_{0}}{2}\left[\bar{I}_{1}-3\right]-\left(1-c_{p}-c_{i}\right) \frac{\mu_{0}}{2}\left[\mathcal{I}_{1}-3\right]$,

where $\mathcal{I}_{1}$ is explicitly given by expression $(47)$ in terms of $c_{p}, c_{i}, \bar{I}_{1}$, and the variables $\bar{\mu}_{0}$, $\mu_{0}$, which are defined implicitly by the system of two coupled nonlinear algebraic equations

$$
\mathcal{F}_{1}\left\{\bar{\mu}_{0}, \mu_{0}\right\}=0, \quad \mathcal{F}_{2}\left\{\bar{\mu}_{0}, \mu_{0}\right\}=\frac{3^{1-\alpha_{1}}}{2} \mu_{1} \mathcal{I}_{1}^{\alpha_{1}-1}+\frac{3^{1-\alpha_{2}}}{2} \mu_{2} \mathcal{I}_{1}^{\alpha_{2}-1}-\frac{\mu_{0}}{2}=0
$$

recall that the function $\mathcal{F}_{1}$ is defined by expression (40).

Parts (a), (b), and (c) of Fig. 18 show stress-deformation results for uniaxial compression, uniaxial tension, and simple shear for particle concentration $c_{p}=0.15$ and three different types of interphases: $(i)$ no interphases $t / r=0,(i i)$ interphases with moderate shear modulus $\mu_{i} / \mu_{m}=5$ and moderate thickness $t / r=0.1$, and (iii) rigid interphases $\mu_{i}=+\infty$ with relatively large thickness $t / r=0.2$. The constitutive stress-deformation relations for the specified loadings are given by

- Uniaxial loading $\left(\bar{\lambda}_{1}=\bar{\lambda}, \bar{\lambda}_{2}=\bar{\lambda}_{3}=\bar{\lambda}^{-1 / 2}\right.$ with $\left.\bar{\tau}_{2}=\bar{\tau}_{3}=0\right)$ :

$$
\bar{S}_{u n}=\bar{\lambda}^{-1} \bar{\tau}_{1}=\frac{\mathrm{d} \bar{\Psi}}{\mathrm{d} \bar{\lambda}}=\bar{\mu}_{0}\left[\bar{\lambda}-\bar{\lambda}^{-2}\right]
$$

- Simple shear $\left(\bar{\lambda}_{1}=\left(\bar{\gamma}+\sqrt{\bar{\gamma}^{2}+4}\right) / 2, \bar{\lambda}_{2}=\bar{\lambda}_{1}^{-1}, \bar{\lambda}_{3}=1\right)$ :

$$
\bar{S}_{s s}=\frac{\mathrm{d} \bar{\Psi}}{\mathrm{d} \bar{\gamma}}=\bar{\mu}_{0} \bar{\gamma}
$$




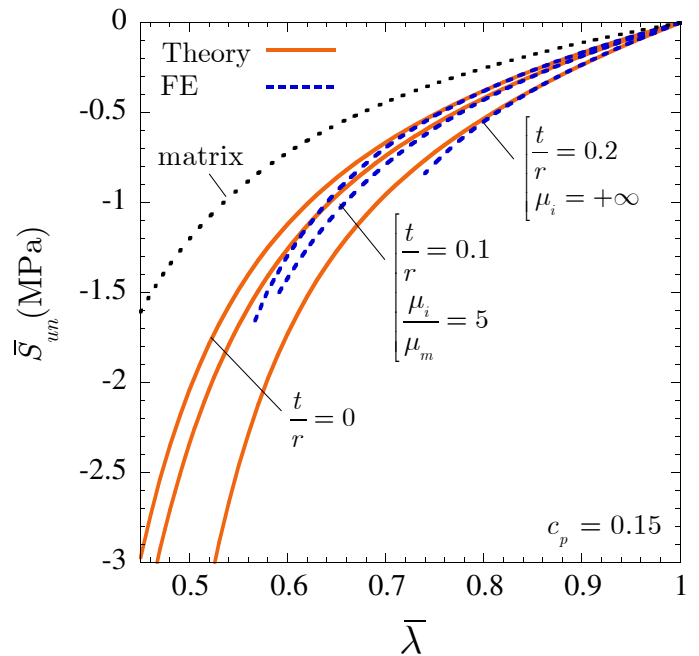

(a)

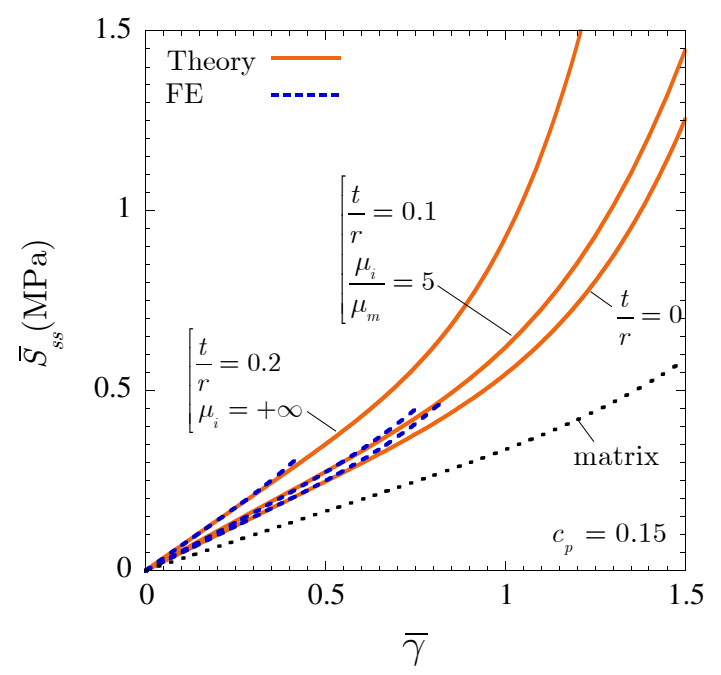

(c)

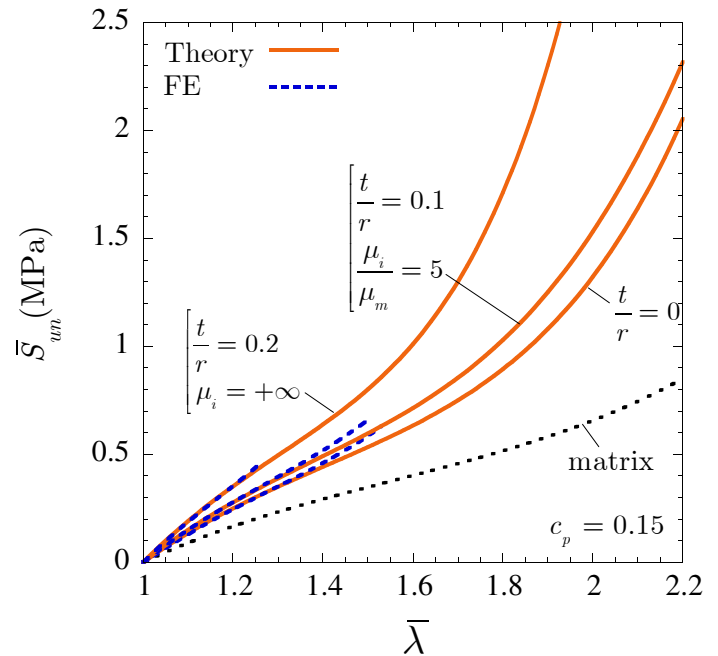

(b)

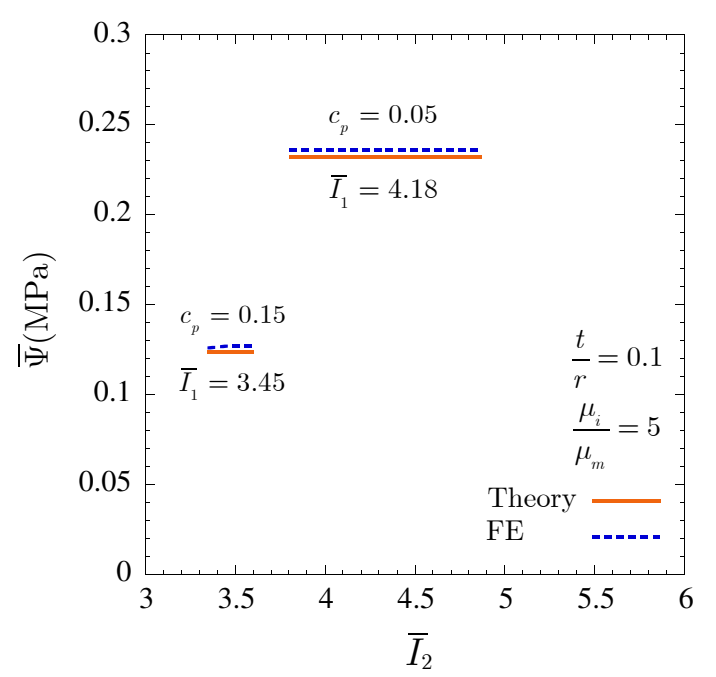

(d)

Figure 18: Macroscopic response of a filled silicone elastomer under: (a) uniaxial compressive, (b) uniaxial tensile, and (c) simple shear loading conditions. Plots are shown for the theoretical stress-deformation results (75), (76) with (73), and corresponding FE simulations for particle concentration $c_{p}=0.15$ and three different types of interphases: (i) no interphases $t / r=0$, (ii) interphases with moderate shear modulus $\mu_{i} / \mu_{m}=5$ and moderate thickness $t / r=0.1$, and (iii) rigid interphases $\mu_{i}=+\infty$ with relatively large thickness $t / r=0.2$. Part (d) of the figure shows comparisons between the effective stored-energy function (73) and corresponding FE results for $t / r=0.1$, $\mu_{i} / \mu_{m}=5, c_{p}=0.05,0.15$ and two fixed values of the first principal invariant $\bar{I}_{1}$, in terms of the second invariant $\bar{I}_{2}$.

where, as above, $\bar{S}_{u n}, \bar{S}_{s s}$ denote first Piola-Kirchhoff stress measures, $\bar{\tau}_{1}, \bar{\tau}_{2}, \bar{\tau}_{3}$ stand for the macroscopic principal Cauchy stresses, and, again, the coefficient $\bar{\mu}_{0}$ is defined implicitly by the system of equations (74). Part (d) of Fig. 18 displays results for the effective stored-energy function (73) for fixed values of the first principal invariant $\bar{I}_{1}=3.45$ for $c_{p}=0.15$ and $\bar{I}_{1}=4.18$ for $c_{p}=0.05$, both for interphases with $t / r=0.1$ and $\mu_{i} / \mu_{m}=5$, in terms of the second invariant $\bar{I}_{2}$. All four parts of Fig. 18 include corresponding FE results. Akin to the preceding Gaussian case, we make no distinction here of whether the particles are of the same or of different sizes since, again, the simulated monodisperse and polydisperse 
microstructures turn out to exhibit practically the same response for particle concentrations below $c_{p}=0.2$ with interphase-thickness-to-particle-radius ratios below $t / r=0.2$.

Similar to the foregoing, Figs. 18(a) through (c) illustrate that both the addition of particles as well as the presence of interphases have a profound stiffening effect for all deformations. They also show that the theoretical and FE results are in fairly good qualitative and quantitative agreement for all loading conditions, especially for small and moderate deformations. For large enough deformations at which the limiting chain extensibility of the silicone elastomer comes into effect, the analytical results are consistently softer - as expected from their variational construction process (see remark $(v)$ in Section 4.2) - than their FE counterparts. Fig. 18(d) shows that the FE results for the filled silicone elastomer, much like those for the filled Guassian elastomer, are approximately independent of the second macroscopic invariant $\bar{I}_{2}$, in functional accord with the proposed theory.

\section{Comparisons with experimental data and final comments}

In the sequel, we deploy the theoretical result (46) to scrutinize a series of representative experimental data available in the literature. The objective is to illustrate the use of the proposed theory and to showcase its ability not only to describe the macroscopic response of real filled elastomers but also, and more critically, to unveil how the various microscopic quantities contribute individually to such a macroscopic response.

We begin by considering the experimental data of Mullins and Tobin (1965), Omnès et al. (2008), and Smallwood (1944) for the macroscopic response in the small-deformation regime of polyisoprene rubber reinforced with a random and isotropic distribution of carbon black particles. Specifically, Fig. 19(a) shows the effective initial shear modulus $\bar{\mu}$, normalized by the shear modulus of the underlying polyisoprene matrix $\mu_{m}$, as a function of the concentration of carbon black $c_{p}$. The discrete symbols (empty circles, triangles, and solid circles) correspond to the experimental data, while the solid lines stand for the theoretical predictions.

The results of Mullins and Tobin (1965) correspond to specimens with a well-dispersed distribution of roughly spherical aggregates of carbon black that had a relatively large average radius of about $200 \mathrm{~nm}(c f$. Fig. 1 in their paper). By way of swelling experiments, these authors were able to conclude that the elastic properties of the polyisoprene rubber were essentially unmodified by the presence of fillers, but provided no insight into the amount or type of bound rubber surrounding them. Given this partial information, at the level of the theoretical result (46), it is reasonable to assume that the shear modulus of the polyisoprene rubber matrix is identical to that of the polyisoprene rubber when synthesized in the absence of carbon black, namely, $\mu_{m}=0.44 \mathrm{MPa}$. And that the content of interphases is comparatively negligible to that of the relatively large fillers so that $c_{i}=0$, or equivalently, $t / r=0$. Figure 19(a) shows that the theoretical predictions based on these inputs are in fairly good agreement with the experimental measurements of Mullins and Tobin (1965), save for the filled elastomer with the largest particle concentration $c_{p}=0.2$.

The specimens studied by Omnès et al. (2008) were also comprised of a well-dispersed distribution of roughly spherical carbon black aggregates but of much smaller size, in the order of $30 \mathrm{~nm}$ in average radius (cf. Figs. 1(a) and (c) in their paper). As opposed to Mullins and Tobin (1965), Omnès et al. (2008) did find that the elastic properties of the polyisoprene rubber were somewhat modified by the presence of carbon black. Unfortunately, no concrete measurements of this change were reported nor details about the amount or type of bound rubber surrounding the fillers provided. In view of this partial information, at the level of the theoretical result (46), we assume, as a first-order approximation, that the shear modulus of the polyisoprene rubber matrix is identical to that of the polyisoprene rubber when synthesized in the absence of carbon black, which in this case was reported as $\mu_{m}=0.53 \mathrm{MPa}$. Also, based on the recent experiments of $\mathrm{Qu}$ et al. (2011), we take the shear modulus of the interphases to be 10 times stiffer than that of the matrix, namely, $\mu_{i}=10 \times \mu_{m}=5.3 \mathrm{MPa}$. Making use of these values, we then select the thickness of the interphases by fitting the experimentally measured effective shear modulus. As shown in Fig. 19(a), it is found that an interphase-thickness-to-particle-radius ratio of $t / r=0.37$ - corresponding to interphase thicknesses in the order of $t=0.37 \times 30 \mathrm{~nm}=11.1 \mathrm{~nm}$ - renders good agreement with the data of Omnès et al. (2008).

Regarding the classical data reported by Smallwood (1944) for carbon black filled rubber, no microscopic information is known other than the concentration of particles $c_{p}$. Assuming that the elastic properties of the 


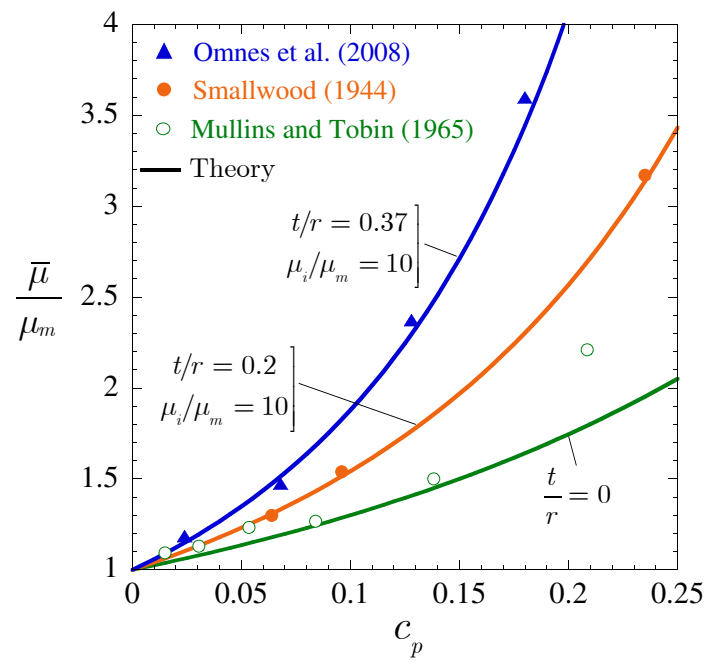

(a)

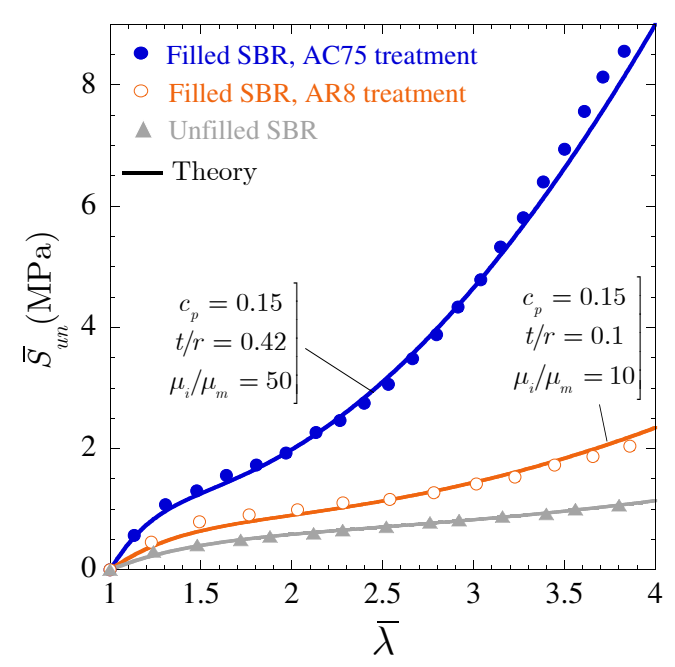

(b)

Figure 19: Comparisons of the proposed theory with experimental data. Part (a) shows the experimental results of Mullins and Tobin (1965), Omnès et al. (2008), and Smallwood (1944) for the effective initial shear modulus $\bar{\mu}$ of polyisoprene rubber filled with carbon black particles, normalized by the initial shear modulus of the corresponding rubber $\mu_{m}$, as a function of the concentration of carbon black $c_{p}$. Part (b) shows the experimental results of Ramier (2004) for the uniaxial tensile stress-stretch response of SBR rubber filled with silica particles, at concentration $c_{p}=0.15$, with two different types of chemical treatments, labeled as AC75 and AR8. In both parts of the figure, the discrete symbols (empty circles, triangles, and solid circles) correspond to the experimental measurements, while the solid lines stand for the theoretical predictions.

rubber making up the matrix are unaffected by the presence of carbon black, and that the shear modulus of the interphases is 10 times stiffer than that of the matrix, the theoretical result (46) can be seen to describe fairly accurately the measurements of Smallwood (1944) by choosing an interphase-thickness-to-particleradius ratio of $t / r=0.2$.

We now turn to examine the experimental data of Ramier (2004) for the large-deformation response under uniaxial tension of SBR rubber filled with a random and isotropic distribution of silica particles. Figure 19(b) shows the measured uniaxial (first Piola-Kirchhoff) stress $\bar{S}_{\text {un }}$ as a function of the applied stretch $\bar{\lambda}$ for two specimens with the same concentration of silica particles, $c_{p}=0.15$, which have undergone two different chemical treatments, labeled as AC75 and AR8. To aid the discussion, Fig. 19(b) also shows the uniaxial stress-stretch response for the unfilled SBR rubber. Akin to Fig. 19(a), the discrete symbols (empty circles, triangles, and solid circles) correspond to the experimental data, while the solid lines stand for the theoretical predictions.

Irrespective of the chemical treatment of the particles, the results of Ramier (2004) correspond to specimens with a well-dispersed distribution of roughly spherical aggregates of silica that had an average radius of about $40 \mathrm{~nm}$ (see Chapter III in that reference). It is unclear to what extent the presence of silica with either treatment, AC75 or AR8, affected the elastic properties of the SBR rubber. On the other hand, the AC75 treatment was expected, by design, to promote the formation of thicker and stiffer interphases than the AR8 treatment. The much stiffer macroscopic response exhibited by the specimen with the AC75-treated silica is consistent with this expectation.

In computing the theoretical predictions for the data of Ramier (2004), given the above-outlined partial information, we assume that the SBR rubber is, to a first approximation, unaffected by the presence of silica. Moreover, we take the SBR rubber to be characterized by the Lopez-Pamies stored-energy function (69) with material parameters $\mu_{1}=0.3734 \mathrm{MPa}, \mu_{2}=0.0425 \mathrm{MPa}, \alpha_{1}=0.3841, \alpha_{2}=1.7767$. Figure 19(b) shows that this model describes accurately the experimentally measured response of the SBR rubber, at 
least for uniaxial tension. We shall further assume, again, based on the recent experiments due to Qu et al. (2011), that the interphases in the specimen with AR8-treated silica are 10 times stiffer than the matrix, $\mu_{i}=10 \times \mu_{m}=10 \times\left(\mu_{1}+\mu_{2}\right)=4.16 \mathrm{MPa}$, whereas the interphases in the specimen with AC75-treated silica are 50 times stiffer, $\mu_{i}=50 \times \mu_{m}=50 \times\left(\mu_{1}+\mu_{2}\right)=20.80 \mathrm{MPa}$. Since there is no experimental evidence available regarding the sizes of the interphases, we select them here by fitting the theory to the experimental stress-stretch responses. As shown by Fig. 19(b), an interphase-thickness-to-particle-radius ratio of $t / r=0.1$ - corresponding to interphase thicknesses in the order of $t=0.1 \times 30 \mathrm{~nm}=3 \mathrm{~nm}-$ leads to a good agreement with the AR8 data. On the other hand, an interphase-thickness-to-particle-radius ratio of $t / r=0.42$ - corresponding to interphase thicknesses in the order of $t=0.42 \times 30 \mathrm{~nm}=12.6 \mathrm{~nm}$ - renders good agreement with the AC75 data.

In summary, the above comparisons with experiments indicate that the proposed microscopic field theory is able to describe and explain the macroscopic response of filled elastomers at finite deformations. In particular, the comparisons indicate that the reinforcement granted by interphases is comparable to that granted by the fillers themselves. These results make it plain that some knowledge of the geometry and constitutive properties of the underlying interphases - and not just the fillers — in elastomers is of the essence to be able to predict, and thus also to design from the bottom up, the macroscopic behavior of filled elastomers.

As a final comment, we remark that in addition to the hydrodynamic and interphasial reinforcement effects, the presence of occluded rubber in filled elastomers may also provide additional reinforcement. Occluded rubber refers to the regions of elastomer that are entrapped by the agglomeration of filler particles. To a first approximation, because of its shielding from the rest of the elastomer, its constitutive behavior can be idealized as rigid. In this case, the presence of occluded rubber can be accounted for by the proposed theory by simply reinterpreting $c_{p}$ as the combined concentration of fillers and occluded rubber.

\section{Acknowledgements}

Support for this work by the National Science Foundation through Grants CMMI-1219336, CMMI1349535, CMMI-1437535, and by the Civil and Environmental Engineering Department at the University of Illinois through the CEE Innovation Grant Program is gratefully acknowledged.

\section{Appendix A. Solution for the single-particle problem in the small-deformation limit}

In this appendix, we work out the elasticity solution for the single-particle problem formulated in Section 3 from which the effective shear modulus (16) is determined.

We find it convenient to begin by considering the boundary-value problem of an isotropic incompressible multicoated sphere occupying the domain $\Omega=\left\{\mathbf{X}:|\mathbf{X}| \leq r_{m}\right\}$, made up of a core (the particle) with initial shear modulus $\mu_{p}$, an inner shell (the interphase) with initial shear modulus $\mu_{i}$, and an outer shell (the matrix) with initial shear modulus $\mu_{m}$, that is subjected to the affine simple shear deformation $\mathbf{x}=\overline{\mathbf{F}} \mathbf{X}$ with $\overline{\mathbf{F}}=\mathbf{I}+\bar{\gamma} \mathbf{e}_{1} \otimes \mathbf{e}_{2}$ on its boundary $\partial \Omega=\left\{\mathbf{X}:|\mathbf{X}|=r_{m}\right\}$. The heterogeneous shear modulus of such a sphere can be written in the compact form

$$
\mu(\mathbf{X})=\left[1-\theta_{p}(\mathbf{X})-\theta_{i}(\mathbf{X})\right] \mu_{m}+\theta_{p}(\mathbf{X}) \mu_{p}+\theta_{i}(\mathbf{X}) \mu_{i}
$$

where $\theta_{p}(\mathbf{X})=1$ if $|\mathbf{X}| \leq r$ and zero otherwise, $\theta_{i}(\mathbf{X})=1$ if $r \leq|\mathbf{X}| \leq r+t$ and zero otherwise. Here, $r$ and $t$ stand, respectively, for the initial radius of the core and the thickness of the inner shell. In the limit as the applied amount of shear $\bar{\gamma} \rightarrow 0$, the equilibrium displacement field $\mathbf{u}=\mathbf{x}-\mathbf{X}$ that minimizes the total elastic energy $\bar{\Psi}$ (per unit undeformed volume) of the sphere takes the form $\mathbf{u}=u_{1}(\mathbf{X}) \mathbf{e}_{1}+u_{2}(\mathbf{X}) \mathbf{e}_{2}+u_{3}(\mathbf{X}) \mathbf{e}_{3}$ 
with

$$
\begin{aligned}
& u_{1}(\mathbf{X})=\bar{\gamma}\left[B^{(1)}+B^{(2)} \frac{r^{5}}{|\mathbf{X}|^{5}}+B^{(3)} \frac{|\mathbf{X}|^{2}}{r^{2}}\right] X_{2}-\bar{\gamma}\left[5 B^{(2)} \frac{r^{5}}{|\mathbf{X}|^{5}}+\frac{4}{5} B^{(3)} \frac{|\mathbf{X}|^{2}}{r^{2}}-3 B^{(4)} \frac{r^{3}}{|\mathbf{X}|^{3}}\right] \frac{X_{1}^{2} X_{2}}{|\mathbf{X}|^{2}}, \\
& u_{2}(\mathbf{X})=\bar{\gamma}\left[B^{(1)}+B^{(2)} \frac{r^{5}}{|\mathbf{X}|^{5}}+B^{(3)} \frac{|\mathbf{X}|^{2}}{r^{2}}\right] X_{1}-\bar{\gamma}\left[5 B^{(2)} \frac{r^{5}}{|\mathbf{X}|^{5}}+\frac{4}{5} B^{(3)} \frac{|\mathbf{X}|^{2}}{r^{2}}-3 B^{(4)} \frac{r^{3}}{|\mathbf{X}|^{3}}\right] \frac{X_{1} X_{2}^{2}}{|\mathbf{X}|^{2}}, \\
& u_{3}(\mathbf{X})=-\bar{\gamma}\left[5 B^{(2)} \frac{r^{5}}{|\mathbf{X}|^{5}}+\frac{4}{5} B^{(3)} \frac{|\mathbf{X}|^{2}}{r^{2}}-3 B^{(4)} \frac{r^{3}}{|\mathbf{X}|^{3}}\right] \frac{X_{1} X_{2} X_{3}}{|\mathbf{X}|^{2}},
\end{aligned}
$$

and

$$
B^{(I)}=\left[1-\theta_{p}(\mathbf{X})-\theta_{i}(\mathbf{X})\right] B_{m}^{(I)}+\theta_{p}(\mathbf{X}) B_{p}^{(I)}+\theta_{i}(\mathbf{X}) B_{i}^{(I)} \quad(I=1,2,3,4),
$$

to $O(\bar{\gamma})$ (see, e.g., Chapter XI in Love, 1906). By the same token, the corresponding traction field takes the form $\mathbf{t}=\mathrm{t}_{1}(\mathbf{X}) \mathbf{e}_{1}+\mathrm{t}_{2}(\mathbf{X}) \mathbf{e}_{2}+\mathrm{t}_{3}(\mathbf{X}) \mathbf{e}_{3}$ with

$$
\begin{aligned}
\mathrm{t}_{1}(\mathbf{X})= & 2 \mu(\mathbf{X}) \bar{\gamma}\left[B^{(1)}-4 B^{(2)} \frac{r^{5}}{|\mathbf{X}|^{5}}+\frac{8}{5} B^{(3)} \frac{|\mathbf{X}|^{2}}{r^{2}}+\frac{3}{2} B^{(4)} \frac{r^{3}}{|\mathbf{X}|^{3}}\right] \frac{X_{2}}{|\mathbf{X}|}+ \\
& 2 \mu(\mathbf{X}) \bar{\gamma}\left[20 B^{(2)} \frac{r^{5}}{|\mathbf{X}|^{5}}-\frac{19}{5} B^{(3)} \frac{|\mathbf{X}|^{2}}{r^{2}}-12 B^{(4)} \frac{r^{3}}{|\mathbf{X}|^{3}}\right] \frac{X_{1}^{2} X_{2}}{|\mathbf{X}|^{3}}, \\
\mathrm{t}_{2}(\mathbf{X})= & 2 \mu(\mathbf{X}) \bar{\gamma}\left[B^{(1)}-4 B^{(2)} \frac{r^{5}}{|\mathbf{X}|^{5}}+\frac{8}{5} B^{(3)} \frac{|\mathbf{X}|^{2}}{r^{2}}+\frac{3}{2} B^{(4)} \frac{r^{3}}{|\mathbf{X}|^{3}}\right] \frac{X_{1}}{|\mathbf{X}|}+ \\
& 2 \mu(\mathbf{X}) \bar{\gamma}\left[20 B^{(2)} \frac{r^{5}}{|\mathbf{X}|^{5}}-\frac{19}{5} B^{(3)} \frac{|\mathbf{X}|^{2}}{r^{2}}-12 B^{(4)} \frac{r^{3}}{|\mathbf{X}|^{3}}\right] \frac{X_{1} X_{2}^{2}}{|\mathbf{X}|^{3}}, \\
\mathrm{t}_{3}(\mathbf{X})= & 2 \mu(\mathbf{X}) \bar{\gamma}\left[20 B^{(2)} \frac{r^{5}}{|\mathbf{X}|^{5}}-\frac{19}{5} B^{(3)} \frac{|\mathbf{X}|^{2}}{r^{2}}-12 B^{(4)} \frac{r^{3}}{|\mathbf{X}|^{3}}\right] \frac{X_{1} X_{2} X_{3}}{|\mathbf{X}|^{3}} .
\end{aligned}
$$

In these expressions, $B_{m}^{(I)}, B_{p}^{(I)}$, and $B_{i}^{(I)}(I=1,2,3,4)$ are constants to be determined from the boundary conditions applied at $\partial \Omega=\left\{\mathbf{X}:|\mathbf{X}|=r_{m}\right\}$ and from the continuity of the displacement and traction fields at the surfaces of material discontinuity $\Gamma_{p}=\{\mathbf{X}:|\mathbf{X}|=r\}$ and $\Gamma_{i}=\{\mathbf{X}:|\mathbf{X}|=r+t\}$. Upon recognizing that $B_{p}^{(2)}=B_{p}^{(4)}=0$, these conditions lead to a system of 10 linear algebraic - and thus readily solvable - equations for the 10 unknowns $B_{p}^{(1)}, B_{p}^{(3)}, B_{i}^{(1)}, B_{i}^{(2)}, B_{i}^{(3)}, B_{i}^{(4)}, B_{m}^{(1)}, B_{m}^{(2)}, B_{m}^{(3)}, B_{m}^{(4)}$. The explicit expressions for these constants, in terms of the shear moduli $\mu_{p}, \mu_{i}, \mu_{m}$, and the lengths $r, t, r_{m}$, are fairly cumbersome and thus not reported here. Having determined the equilibrium displacement field $\mathbf{u}$ over the entire sphere, its total elastic energy (per unit undeformed volume) is simply given by

$$
\bar{\Psi}=\frac{1}{2|\Omega|} \int_{\partial \Omega} \mathbf{t} \cdot \mathbf{u} \mathrm{d} S=\left[B_{m}^{(1)}+\frac{42 r_{m}^{2}}{50 r^{2}} B_{m}^{(3)}-\frac{45 r^{3}}{50 r_{m}^{3}} B_{m}^{(4)}\right] \mu_{m} \bar{\gamma}^{2} .
$$

Now, in the limit when the radius of the sphere is taken to be infinitely large and the core is taken to be rigid, as $r_{m} \rightarrow+\infty$ and $\mu_{p} \rightarrow+\infty$, the total elastic energy (81) reduces to the effective stored-energy function for the single-particle problem formulated in Section 3, in the limit of small deformations and for the specific case of simple shear loading, namely,

$$
\bar{\Psi}=\bar{\mu}^{\mathrm{dil}} \operatorname{tr} \bar{\varepsilon}^{2}=\frac{\bar{\mu}^{\mathrm{dil}}}{2} \bar{\gamma}^{2} .
$$

The solution (16) for $\bar{\mu}^{\text {dil }}$ provided in the main body of the text follows by comparing (81) with (82). As also remarked in the main body of the text, the product $\mu_{p} B_{p}^{(3)}$ does not vanish in this limit and thus the stress field within the particle - contrary to the classical solution (Eshelby, 1957) without an interphase is not uniform. 


\section{References}

[1] ABAQUS Version 6.11 Documentation, Dassault Systèmes Simulia Corp., Providence, RI, USA, 2011.

[2] Arruda, E.M., Boyce, M.C., 1993. A three-dimensional constitutive model for the large stretch behavior of rubber elastic materials. J. Mech. Phys. Solids 41, 389-412.

[3] Beatty, M.F., 2003. An average-stretch full-network model for rubber elasticity. J. Elasticity 70, 65-86.

[4] Berriot, J., Montes, H., Lequeux, F., Long, D., Sotta, P., 2002. Evidence of the shift of the glass transition near the particles in silica-filled elastomers. Macromolecules 35, 9756-9762.

[5] deBotton, G., Shmuel, G., 2010. A new variational estimate for the effective response of hyperelastic composites. J. Mech. Phys. Solids 58, 466-483.

[6] Deepalekshmi, P., Thomas, S., 2014. Non-Linear Viscoelasticity of Rubber Composites and Nanocomposites. Springer.

[7] Diani, J., Fayolle, B., Gilormini, P., 2009. A review on the Mullins effect. European Polymer Journal 45, 601-612.

[8] Diani, J., Gilormini, P., Merckel, Y., Vion-Loisel, F., 2013. Micromechanical modeling of the linear viscoelasticity of carbon-black filled styrene butadiene rubbers: the role of the rubber-filler interphase. Mechanics of Materials 59, 65-72.

[9] Diani, J., Gilormini, P., 2014. Using a pattern-based homogenization scheme for modeling the linear viscoelasticity of nano-reinforced polymers with an interphase. J. Mech. Phys. Solids 63, 51-61, 2014.

[10] Duan, H.L., Jiao, Y., Yi, X., Huang, Z.P., Wang, J., 2006. Solutions of inhomogeneity problems with graded shells and application to core-shell nanoparticles and composites. J. Mech. Phys. Solids 54, 1401-1425.

[11] Einstein, A., 1906. Eine neue bestimmung der moleküldimensionen [A new determination of molecular dimensions]. Annalen der Physik 324, 289-306.

[12] Eshelby, J.D., 1957. The determination of the elastic field of an ellipsoidal inclusion and related problems. Proc. R. Soc. Lond. A 241, 376-396.

[13] Fukahori, Y., 2007. Generalized concept of the reinforcement of elastomers. Part 1: Carbon black reinforcement of rubbers. Rubber Chemistry and Technology 80, 701-725.

[14] Gent, A.N., 1996. A new constitutive relation for rubber. Rubber Chem. Technol. 69, 59-61.

[15] Gent, A.N., Park, B., 1984. Failure processes in elastomers at or near a rigid inclusion. J. Mater. Sci. 19, $1947-1956$.

[16] Gusev, A.A., 1997. Representative volume element size for elastic composites: a numerical study. J. Mech. Phys. Solids $45,1449-1459$

[17] Guth, E., 1945. Theory of filler reinforcement. Journal of Applied Physics 16, 20-25.

[18] Hashin, Z., 1991. Composite materials with viscoelastic interphase: creep and relaxation. Mechanics of Materials 11, 135-148.

[19] Heinrich, G., Klüppel, M., Vilgis, T.A., 2002. Reinforcement of elastomers. Current Opinion in Solid State and Materials Science 6, 195-203.

[20] Herve, E., Zaoui, A., 1993. N-layered inclusion-based micromechanical modelling. Int. J. Eng. Sci. 31, 1-10.

[21] Hill, R., 1972. On constitutive macrovariables for heterogeneous solids at finite strain. Proc. R. Soc. Lond. A 326, $131-147$.

[22] Leblanc, J.L., 2010. Filled Polymers: Science and Industrial Applications. CRC Press.

[23] Lopez-Pamies, O., 2010a. A new $I_{1}$-based hyperelastic model for rubber elastic materials. Comptes Rendus Mecanique $338,3-11$.

[24] Lopez-Pamies, O., 2010b. An exact result for the macroscopic response of particle-reinforced Neo-Hookean solids. Journal of Applied Mechanics 77, 021016.

[25] Lopez-Pamies, O. 2014. Elastic dielectric composites: Theory and application to particle-filled ideal dielectrics. J. Mech. Phys. Solids 64, 61-82.

[26] Lopez-Pamies, O., Goudarzi, T., Nakamura, T., 2013a. The nonlinear elastic response of suspensions of rigid inclusions in rubber: I - An exact result for dilute suspensions. J. Mech. Phys. Solids 61, 1-18.

[27] Lopez-Pamies, O., Goudarzi, T., Danas, K., 2013b. The nonlinear elastic response of suspensions of rigid inclusions in rubber: II - A simple explicit approximation for finite-concentration suspensions. J. Mech. Phys. Solids 61, 19-37.

[28] Love, A.E.H., 1906. A treatise on the mathematical theory of elasticity. Cambridge University Press.

[29] Michel, J.C., Moulinec, H., Suquet, P., 1999. Effective properties of composite materials with periodic microstructure: a computational approach. Comput. Methods Appl. Mech. Engrg. 172, 109-143.

[30] Mikata, T., Taya, M., 1985. Stress field in and around a coated short fiber in an infinite matrix subjected to uniaxial and biaxial loadings. J. Appl. Mech. 52, 19-24.

[31] Mullins, L., Tobin, N.R., 1965. Stress softening in rubber vulcanizates. Part I. Use of a strain amplification factor to describe the elastic behavior of filler-reinforced vulcanized rubber. Journal of Applied Polymer Science 9, $2993-3009$.

[32] Norris, A.N., 1985. A differential scheme for the effective moduli of composites. Mech. Materials 4, 1-16.

[33] Omnès, B., Thuillier, S., Pilvin, P., Grohens, Y., Gillet, S., 2008. Effective properties of carbon black filled natural rubber: Experiments and modeling. Composites Part A: Applied Science and Manufacturing 39, 1141-1149.

[34] Ogden, R.W., 1978. Extremum principles in non-linear elasticity and their application to composites - I Theory. Int. J. Solids Struct. 14, 265-282.

[35] Ponte Castañeda, P., 1991. The effective mechanical properties of nonlinear isotropic composites. J. Mech. Phys. Solids $39,45-71$.

[36] Qiu, Y.P., Weng, G.J., 1991. Elastic moduli of thickly coated particle and fiber-reinforced composites. J. Appl. Mech. 58, 388-398.

[37] Qu, M., Deng, F., Kalkhoran, S.M., Gouldstone, A., Robisson, A., Van Vliet, K.J., 2011. Nanoscale visualization and multiscale mechanical implications of bound rubber interphases in rubber-carbon black nanocomposites. Soft Matter 7 , 1066-1077. 
[38] Ramier, J., 2004. Comportement mécanique d'élastomères chargés, influence de l'adhésion charge-polymère, influence de la morphologie. Ph. D. Dissertation, Institut National des Sciences Appliquées de Lyon, France.

[39] Roscoe, R., 1973. Isotropic composites with elastic or viscoelastic phases: General bounds for the moduli and solutions for special geometries. Rheologica Acta 12, 404-411.

[40] Schöberl, J., 1997. Netgen an advancing front 2d/3d-mesh generator based on abstract rules. Computing and Visualization in Science 1, 41-52.

[41] Segurado, J., Llorca, J., 2002. A numerical approximation to the elastic properties of sphere-reinforced composites. J. Mech. Phys. Solids 50, 2107-2121.

[42] Smallwood, H.M., 1944. Limiting law of the reinforcement of rubber. Journal of Applied Physics 15, 758-766.

[43] Spring, D.W., Paulino, G.H., 2014. A growing library of three-dimensional cohesive elements for use in ABAQUS. Engineering Fracture Mechanics 126, 190-216.

[44] Walpole, J.W., 1978. A coated inclusion in an elastic medium. Math. Proc. Camb. Phil. Soc. 83, 495-506.

[45] Talbot, D.R.S., Willis, J.R., 1985. Variational principles for inhomogeneous nonlinear media. IMA J. Appl. Math. 35, $39-54$.

[46] Willis, J.R., 1977. Bounds and self-consistent estimates for the overall properties of anisotropic composites. J. Mech. Phys. Solids 25, 185-202.

[47] Willis, J.R., 1994. Upper and lower bounds for the properties of nonlinear composites. Mater. Sci. Eng. A $175,7-14$.

[48] Willis, J.R., Acton, J.R., 1976. The overall elastic moduli of a dilute suspension of spheres. Q. J. Mechanics Appl. Math. 29, 163-177.

[49] Zee, L., Sternberg, E., 1983. Ordinary and strong ellipticity in the equilibrium theory of incompressible hyperelastic solids. Arch. Rat. Mech. Analysis 83, 53-90.

[50] Zhang, H., Scholz, A.K., de Crevoisier, J., Vion-Loisel, F., Besnard, G., Hexemer, A., Brown, H.R., Kramer, E.J., Creton, C., 2012. Nanocavitation in carbon black filled styrenebutadiene rubber under tension detected by real time small angle X-ray scattering. Macromolecules 45, 1529-1543. 